\title{
Assessment of a Takagi-Sugeno-Kang fuzzy model assembly for examination of polyphasic loglinear allometry
}

\author{
Hector A Echavarria-Heras ${ }^{\text {Corresp., } 1}$, Juan R Castro-Rodriguez ${ }^{2}$, Cecilia Leal-Ramirez ${ }^{1}$, Enrique Villa-Diharce ${ }^{3}$ \\ ${ }^{1}$ Departamento de Ecología, Centro de Investigación Científica y de Estudios Superiores de Ensenada, Ensenada, Baja California, México \\ 2 Facultad de Ciencias Químicas e Ingeniería, Universidad Autónoma de Baja California, Tijuana, Baja California, México \\ 3 Departamento de Estadística Aplicada, Centro de Investigacion en Matematicas, Guanajuato, Guanajuato, México \\ Corresponding Author: Hector A Echavarria-Heras \\ Email address: heheras@icloud.com
}

Background. The traditional allometric analysis relies on log- transformation to contemplate linear regression in geometrical space then retransforming to get Huxley's model of simple allometry. Views assert this induces bias endorsing multi-parameter complex allometry forms and nonlinear regression in arithmetical scales. Defenders of traditional approach deem it necessary since generally organismal growth is essentially multiplicative. Then keeping allometry as originally envisioned by Huxley requires a paradigm of polyphasic loglinear allometry. A Takagi-Sugeno-Kang fuzzy model assembles a mixture of weighted sub models. This allows direct identification of break points for transition between phases. Then, this paradigm is seamlessly appropriate for efficient allometric examination of polyphasic loglinear allometry patterns. Here, we explore its suitability. Methods. Present fuzzy model embraces firing strength weights from Gaussian membership functions and linear consequents. Weights are identified by subtractive clustering and consequents through recursive least squares or maximum likelihood. Intersection of firing strength factors set criterion to estimate breakpoints. A multiparameter complex allometry model follows by adapting firing strengths by composite membership functions and linear consequents in arithmetical space. Results. TakagiSugeno-Kang surrogates adapted complexity depending on analyzed data set.

Retransformation results conveyed reproducibility strength of similar proxies identified in arithmetical space. Breakpoints were straightforwardly identified. Retransformed form implies complex allometry as a generalization of Huxley's power model involving covariate depending parameters. Huxley reported a breakpoint in the log-log plot of chela mass vs body mass of fiddler crabs (Uca pugnax), attributed to a sudden change in relative growth of the chela approximately when crabs reach sexual maturity. G.C. Packard implied this breakpoint as putative. However, according to present fuzzy methods existence of a break point in Huxley's data could be validated. Conclusions. Offered scheme bears reliable 
analysis of zero intercept allometries based on geometrical space protocols. Endorsed affine structure accommodates either polyphasic or simple allometry if whatever turns required. Interpretation of break points characterizing heterogeneity is intuitive. Analysis can be achieved in an interactive way. This could not have been obtained by relying on customary approaches. Besides, identification of break points in arithmetical scale is straightforward. Present Takagi-Sugeno-Kang arrangement offers a way to overcome the controversy between a school considering a log-transformation necessary and their critics claiming that consistent results can be only obtained through complex allometry models fitted by direct nonlinear regression in the original scales. 
1 Assessment of a Takagi-Sugeno-Kang fuzzy model

2 assembly for examination of polyphasic loglinear

3 allometry

4

5

Héctor Echavarría-Heras ${ }^{1}$, Juan Ramón Castro-Rodríguez², Cecilia Leal-Ramírez ${ }^{1}$, Enrique

Villa-Diharce ${ }^{3}$.

7

8 'Departamento de Ecología, Centro de Investigación Científica y de Estudios Superiores de

9 Ensenada, Ensenada, Baja California, México.

$10{ }^{2}$ Facultad de Ciencias Químicas e Ingeniería, Universidad

11 Autónoma de Baja California, Tijuana, Baja California, México.

12 '3epartamento de Estadística Aplicada, Centro de Investigación en

13 Matemáticas, Guanajuato, Guanajuato, México.

14

Corresponding Author:

Héctor Echavarría-Heras ${ }^{1}$

Carretera Ensenada-Tijuana No. 3918, Zona Playitas, Código Postal 22860, Apdo. Postal 360, Ensenada, Baja California, México.

Email address: heheras@icloud.com

\section{Abstract}

Background. The traditional allometric analysis relies on log- transformation to contemplate

linear regression in geometrical space then retransforming to get Huxley's model of simple

allometry. Views assert this induces bias endorsing multi-parameter complex allometry forms and nonlinear regression in arithmetical scales. Defenders of traditional approach deem it necessary since generally organismal growth is essentially multiplicative. Then keeping allometry as originally envisioned by Huxley requires a paradigm of polyphasic loglinear allometry. A Takagi-Sugeno-Kang fuzzy model assembles a mixture of weighted sub models.

This allows direct identification of break points for transition between phases. Then, this 
31 paradigm is seamlessly appropriate for efficient allometric examination of polyphasic loglinear

32 allometry patterns. Here, we explore its suitability.

33 Methods. Present fuzzy model embraces firing strength weights from Gaussian membership

34 functions and linear consequents. Weights are identified by subtractive clustering and

35 consequents through recursive least squares or maximum likelihood. Intersection of firing

36 strength factors set criterion to estimate breakpoints. A multi-parameter complex allometry

37 model follows by adapting firing strengths by composite membership functions and linear

38 consequents in arithmetical space.

39 Results. Takagi-Sugeno-Kang surrogates adapted complexity depending on analyzed data set.

40 Retransformation results conveyed reproducibility strength of similar proxies identified in

41 arithmetical space. Breakpoints were straightforwardly identified. Retransformed form implies

42 complex allometry as a generalization of Huxley's power model involving covariate depending

43 parameters. Huxley reported a breakpoint in the log-log plot of chela mass $v s$ body mass of

44 fiddler crabs (Uca pugnax), attributed to a sudden change in relative growth of the chela

45 approximately when crabs reach sexual maturity. G.C. Packard implied this break point as

46 putative. However, according to present fuzzy methods existence of a break point in Huxley’s

47 data could be validated.

48 Conclusions. Offered scheme bears reliable analysis of zero intercept allometries based on

49 geometrical space protocols. Endorsed affine structure accommodates either polyphasic or

50 simple allometry if whatever turns required. Interpretation of break points characterizing

51 heterogeneity is intuitive. Analysis can be achieved in an interactive way. This could not have

52 been obtained by relying on customary approaches. Besides, identification of break points in

53 arithmetical scale is straightforward. Present Takagi-Sugeno-Kang arrangement offers a way to 
54 overcome the controversy between a school considering a logtransformation necessary and their

55 critics claiming that consistent results can be only obtained through complex allometry models

56 fitted by direct nonlinear regression in the original scales.

57

58

59

60

61

62

63

64

\section{Introduction}

66 Julian Huxley introduced the theory of constant relative growth between a trait $y$ and overall

67 body size $x$ (Huxley, 1924; Huxley, 1932; Strauss \& Huxley, 1993). This paradigm is commonly

68 refereed as Huxley's model of simple allometry and is essentially formulated through the power

69 law $y=\beta x^{\alpha}$ with $\alpha$ identified as the allometric exponent and $\beta$ as the normalization constant. In

70 biology allometric relationships are to within species, as well as, between species (evolutionary

71 allometry) (Houle et al. 2011; Marquet et al. 2005; West \& Brown, 2005; Pélabon et al. 2014).

72 Power function models are also extensively used in other research fields, e.g., physics (Newman,

73 2007), ecology (Harris et al. 2006; Hood 2007) earth and atmospheric sciences (Hills, 2013), and

74 economics (Wang et al. 2015). This has encouraged many research endeavors addressing

75 interpretation of involved parameters, as well as, suitability of analysis method for getting

76 estimates. Indeed, concomitant to Huxley's theory of relative growth is the Traditional Analysis 
77 Method of Allometry (TAMA hereafter). This is, a widespread device to acquire estimates of the 78 parameters $\alpha$ and $\beta$. It contemplates a logarithmic transformation of the original bivariate data in 79 arithmetical scale in order to consider a linear regression model in geometrical space, and then 80 retransforming to acquire Huxley's model of simple allometry in the original scale. This 81 approach implicitly embraces a notion that variability of the response conforms to a pattern of 82 multiplicative growth. On Huxley's elucidation (Huxley, 1932) the intercept $\ln \beta$ of TAMA's line 83 was of no specific biological importance, but the slope $b$ was significant enough as to mean 84 allometry itself. This interpretation has permeated contemporary research to such an extent that 85 many practitioners still consider it to be the valid theoretical perspective for static and ontogenetic allometry (Eberhard, 2009; Houle et al. 2011; Pélabon et al. 2018). But, there are

87 views that assert that a TAMA approach produces inconsistent results, thereby recommending 88 allometric examination by relying instead on nonlinear regression of in the direct scales of data 89 (Packard, 2017a; Packard, 2013; Packard, 2009; Packard \& Birchard, 2008). This is, debatable 90 for defenders of the traditional approach that claim that, as it is conceived in the original 91 theoretical context of allometry, a logarithmic transformation deems necessary in the analysis 92 (Lai et al. 2013, Klingenberg, 1998; Nevill et al. 2005; Kerkhoff \& Enquist, 2009; Xiao et al. 93 2011; White et al. 2012; Ballantyne, 2013; Glazier, 2013; Niklas \& Hammond, 2014; Lemaître et 94 al. 2015; Pélabon et al. 2018). Yet steering further away from Huxley’s perspective on covariation among different traits, other views conceive allometry centered on the covariation between size and shape (Mosimann, 1970; Klingenberg, 2016). From this standpoint, analysis

97 must rely in Multiple Parameter Complex Allometry (MPCA after this) formalizations through 98 all varieties of nonlinear or discontinuous relationships (e.g. Frankino et al. 2010; MacLeod, 99 2014; Bervian et al. 2006; Lovett \& Felder, 1989; Packard 2013). But, adoption of MPCA 
100 approaches nourishes one of the most fundamental discrepancies among schools of allometric 101 examination. Indeed, for advocates of the traditional approach, examination based on MPCA 102 models fitted in arithmetical scale sacrifices appreciation of biological theory in order to 103 privilege statistical correctness (Houle et al. 2011; Lemaître et al. 2015; Pélabon et al. 2018). A 104 way to keep the analysis in geometrical space while amending unreliability of a linearity 105 assumption is conceiving the notion of non-log linear allometry (Packard, 2012b; Strauss \& 106 Huxley, 1993; Echavarria-Heras et al. 2019). As every analytic function can be expanded as a power series, curvature in geometrical space has been addressed through polynomial regression schemes (Kolokotrones et al. 2010; Lemaître et al. 2014; MacLeod, 2010; Glazier et al. 2013; Tidière et al. 2017; Echavarria-Heras et al. 2019). But, besides difficulties related to biological 110 interpretation of a polynomial mean response, this approach maintains a single functional form 111 of the response over the whole covariate range. This could not account for inherent heterogeneity 112 in the logtransformmed response as contemplated in Huxley's theoretical perspective. Certainly, 113 Huxley reported a breakpoint in the $\log -\log$ plot of chela mass $v s$ body mass of fiddler crabs

114 (Uca pugnax). It was attributed to a sudden change in relative growth of the chela approximately 115 when crabs reach sexual maturity (Huxley, 1924; Huxley, 1927; Huxley, 1932). This suggests a 116 slant aimed at adding complexity in geometrical space while keeping the theoretical essence of 117 traditional allometry in the analytical set up. Is this conception that hosts polyphasic loglinear 118 allometry approaches (PLA afterwards) (Packard, 2016; Gerber, Eble \& Neige, 2008; Strauss \& 119 Huxley, 1993; Hartnoll, 1978). PLA characterizes heterogeneity of the logtransformmed 120 response by composing covariate range into sectors separated by break points. Each subdivision 121 associates to a linear sub model. Broken- line regression (Beckman \&Cook, 1979; Ertel \& 122 Fowlkes, 1976; Tsuboi et al. 2018; Ramírez-Ramírez et al. 2019; Muggeo, 2003; Echavarria- 
123 Heras et al. 2019. Forbes \& Lopez (1989) furnish an empirical approach to identification of PLA

124 patterns. Nevertheless, by relying in nonlinear regression this technique requires starting values

125 for the break-point estimation. Therefore, complications set by local maxima, as well as,

126 inferences on estimates could make implementation difficult (Julious, 2001; Muggeo, 2003).

127 The quest for new tools that increase reliability of analytical methods has been always a

128 motivation in research. This drive explains the introduction of hybrid models that merge different

129 techniques with the aim of efficiently addressing complexity (Kimmins et al. 1999; Alur et al.

130 1995; Ajili, 2004; Pozna et al. 2010; Hamilton et al. 2017). Particularly, soft computing

131 techniques entail modelling procedures, which are supplemental to customary statistics and

132 probability approaches and that bear tolerance to imprecision, uncertainty, partial truth and

133 approximation (Baldwin et al. 1998). For instance, identification and control of nonlinear

134 systems exemplifies a subject that has greatly benefited by adoption of related hybrid modeling

135 schemes (Bonissone et al. 1999; Kawaji et al. 2002; Vrkalovic et al. 2018; Chen, 2001).

136 Implementation of soft computing protocols include techniques of fuzzy set theory, neural

137 networks, probabilistic reasoning, rough sets, machine learning, and evolutionary computing

138 (Zadeh, 1993; Oduguwa et al. 2005; Bello \& Verdegay, 2012; Ibrahim, 2016; Al-kaysi et al.

139 2017; Herrera \& Lopez, 2010). In this upsurge of nonconventional analytical tools, we can place

140 adaptation of fuzzy logic procedures aimed to lessen parametric uncertainty effects in allometry

141 (Schreer, 1997; Schwetter \& Bertone, 2018; Bitar, 2016; Echavarria-Heras et al. 2018; Näther \&

142 Wälder, 2006; Dechnik-Vazquez et al. 2019).

143 An operating regime based modeling approach offers a structure supporting model adaptation

144 amid an empirical and mechanistic standpoint. Local models valid over restricted domains are

145 combined by smooth interpolation into an overall general output (Johansen \& Foss, 1997). 
146 Therefore, this structure naturally hosts heterogeneity as conceived by PLA. One example of a

147 hybrid-operating regime based modeling is the Takagi-Sugeno-Kang fuzzy model (Sugeno \&

148 Kang, 1988; Takagui \& Sugeno, 1985) (TSK in what follows). This construct composes a fuzzy

149 logic step intended to characterize smoothing weight factors. Then, conventional statistical

150 methods are used to acquire estimates of parameters characterizing sub models. It turns out that

151 the general output of a first order TSK fuzzy model can uniformly approximate any continuous

152 function to arbitrarily high precision (Ying; 1988; Zeng et al. 2000). As we show in this

153 examination, an advantage of TSK over PLA, is that, it can offer convenient non-statistical

154 proxies of break points for transition among phases. Moreover, consideration of sub models of a

155 TSK scheme as TAMA's linear functions in geometrical space not only offers a congruent PLA

156 model, but it could also entail a highly biologically meaningful model of allometry, because it

157 can model the breakpoints while keeping the meanings of allometric exponents as in Huxley's

158 original formulation. The exploration of suitability of the TSK scheme to examine PLA patterns

159 has not been undertaken so here we attempted to fill this gap. In what follows a formulation of

160 PLA by means of a the TSK fuzzy model will be referred as TSK-PLA for short.

161 The outstanding approximation capabilities of a TSK fuzzy entails reliable identification of

162 whatever MPCA functional form renders necessary in arithmetical space (Echavarria-Heras et al.

163 2018). Adaptation of the TSK fuzzy model for that aim will be forward designated by means of

164 the TSK-MPCA abbreviation. As a criterion to evaluate the performance of the TSK-PLA proxy

165 we verified the dependability of linked retransformation results, including break point placement

166 and reproducibility strength of mean response function against corresponding estimations

167 produced via TSK-MPCA. It turns out that proposed TSK-PLA analysis method endorsed

168 reliable identification of heterogeneity of examined allometries. Furthermore, the affine structure 
169 of the present fuzzy protocol can accommodate either complex or simple allometry as required to

170 analyzing the data. Thus, the presented TSK-PLA model can be considered as a general tool for

171 examination of zero intercept allometries. Moreover, from a theoretical standpoint a TSK-PLA

172 representation implies an allometric model in arithmetical space that seemingly fits MPCA. This 173 expresses the response as a generalized power function including scaling parameters expressed

174 as functions of the covariate (Bervian et al. 2011; Echavarria-Heras et al. 2019). But, above all, 175 present fuzzy approach contributes by offering a way of overcoming the controversy between a 176 school considering analysis in geometrical space as a must in allometry, and critics claiming that 177 consistent results can only come along by using a MPCA formulation followed by nonlinear 178 regression protocol in the original scale of data. Interestingly, present TSK-PLA arrangement 179 also contributed on qualitative grounds. Certainly, Huxley reported a breakpoint in the $\log -\log$ 180 plot of chela mass $v s$ body mass of fiddler crabs (Uca pugnax). Packard (2012a) inferred this 181 point was only putative. In his own interpretation, perhaps due to combined effects of a log 182 transformation itself and the format of graphical display of Huxley's data. However, application of present Takagi-Sugeno-Kang protocol supports existence of a break point in Huxley's Uca 184 pugnax $\log -\log$ plot.

185 This paper is organized as follows: In the methods section, we formally explain the steps backing 186 the identification of the offered TSK-PLA scheme. There, we explain why this construct can be 187 considered as a generalized protocol for allometric analysis in geometrical space. We also clarify 188 why the offered TSK model implies a MPCA scheme in arithmetical space. The presentation 189 includes an elucidation of sufficient conditions under what the asymptotic mode of the acquired 190 TSK proxy behaves as the power function in Huxley's model of simple allometry. There, we also 191 suggest a correction factor (CF here after) for bias of retransformation of the regression error that 
192 grants highest reproducibility for derived mean response function in arithmetical space. The

193 results section highlights on the advantages of the present approach over conventional

194 counterparts. A discussion section elaborates on the contribution that our approach bears for the

195 general subject of suitability of analysis method in bivariate allometry. An appendix includes a

196 detailed explanation of the steps involved in the construction and identification of the general

197 form of the addressed TSK models.

198 Materials \& Methods

199 a) Data

200 Allometric examination here mainly relied on a primary data set exhibiting curvature in 201 geometrical space. This composes 10412 measurements of Zostera marina (Eelgrass) leaf

202 biomasses $y$ and corresponding leaf areas $x$ as reported in Echavarria-Heras et al. (2018). For 203 comparison, we also considered data reported in Mascaro et al. (2011) comprising 30 Biomass-

204 Diameter at Breast Height measurements on Metrosideros polymorpha. Analisis also extended to 205 data reported in De Robertis \& Williams (2008) including 29363 Length-Weight measurements 206 on Gadus chalcogrammu. This last data set allowed illustration of the performance of the TSK 207 paradigm in a circumstance where the TAMA protocol is consistent. Finally, we analysed the 208 fitness of the TSK in detecting break points in the log-log plot of chela mass vs body mass of 209 fiddler crabs (Uca pugnax) (Huxley, 1924; Huxley,1932).

210 b) Models

211 b.1) General Formula of Allometry

212 The methods engaged here aim to identification of the suitable form of the allometric function 213 representing the variation of a trait $y$ depending on a descriptor $x$. For that purpose, we firstly 214 introduce the formal framework and the notation convention used through. We assume that a 
215 response $y$ and its covariate $x$ belong to domains $Y$ and $X$ of positive real numbers one to one

216 and with $y$ having a zero limit when $x$ approaches zero. We also consider that there exist a

217 function $w(x, \boldsymbol{p}): X \rightarrow Y$ where $\boldsymbol{p}=\left(p_{1}, \ldots, p_{n}\right)$ is a parameter set, and a concomitant approximation

218 error function $\epsilon(x): X \rightarrow Y$ that combine to model whatever form, the linkage between $x$ and $y$

219 acquires. Moreover, we take on, that such a relationship can be expressed through an additive

220 error description

$221 y=w(x, \boldsymbol{p})+\epsilon(x)$

222 or else through the multiplicative error alternate

$223 y=w(x, \boldsymbol{p}) e^{\epsilon(x)}$

224 In order to get $w(x, \boldsymbol{p})$, we can consider the error term $\epsilon(x)$ as a random variable $\epsilon$. Then,

225 specifications above offer two commonly addressed analysis protocols in allometry. A regression

226 model with additive error in arithmetical scale

$227 y=w(x, \boldsymbol{p})+\epsilon$

228 with $\epsilon$ taken as $\psi$ - distributed with zero mean and variance generally expressed as a function $\sigma^{2}$

$229(x)$ of covariate, that is, $\epsilon \sim \psi\left(0, \sigma^{2}(x)\right)$. Fitting equation (3) generally requires direct nonlinear

230 regression protocols. This returns a mean response function $E_{a w}(y \mid x)=w(x, \boldsymbol{p})$. For the sake of

231 facilitating comparison aims in further developments, this $a$ subscript will be maintained to

232 typify a mean response function gotten by means of identification protocols applied in

233 arithmetical space.

234 A second procedure circumscribes to the multiplicative error model of equation (2) and relies in

235 a logtransformation procedure in order to consider a parallel regression model in geometrical

236 space. Formally, we contemplate a mapping $(y, x) \rightarrow(v, u)$ such that $u=\ln x$ and $v=\ln y$. This 
237 sets variation domains $U$ and $V$ for $u$ and $v$ to one. We concomitantly have the regression model 238 with additive error in geometrical space

$239 v=v(u, \pi)+\epsilon$,

240 where formally

$241 v(u, \pi)=\ln (w(x, \boldsymbol{p}))$

242 and $\epsilon$ is random variable as specified above. It follows that back-transforming equation (4) to 243 arithmetical space yields,

$244 y=\exp (v(u, \pi)) e^{\epsilon}$.

245 Then, concomitant mean response function is symbolized through $E_{g w}(y \mid x)$ and becomes

$E_{g w}(y \mid x)=\exp (v(u, \pi)) \delta$

247 where $\delta=E\left(e^{\epsilon}\right)$. Notice that in $E_{g w}(y \mid x)$ we have used the notation convention of a subscript

$248 g$ referring to identification of $w(x, \boldsymbol{p})$ based on the regression model of equations (4) and (5).

249 The CF $\delta$ above provides the necessary adjustment for bias of retransformation of the regression 250 error $\epsilon$ (Mascaro et al. 2011; Baskerville, 1972; Newman, 1993). Assuming $\epsilon \sim N\left(0, \sigma^{2}\right)$ sets $e^{\epsilon}$ 251 to be lognormally distributed. Then, CF becomes

$252 \delta=e^{\sigma^{2} / 2}$.

253 But, Newman (1993) asserts that whenever $\epsilon$ is not normally distributed, $\delta$ is given by the

254 smearing estimate of bias of Duan (1983). Nevertheless, in some settings this nonparametric

255 form can produce bias overcompensation (Manning, 1998; Smith, 1993; Koch \& Smillie, 1986).

256 Zeng \& Tang (2011a) propose an alternate nonparametric form of $\delta$ namely

$257 \delta=1+\sigma^{2} / 2$ 
258 Actually, $\delta$ given this way corresponds to a three terms partial sum approximation of the power

259 series expression of $E\left(e^{\epsilon}\right)$ assuming $E(\epsilon)=0$. By the same token, Echavarria-Heras et al. (2019)

260 suggest a representation for $\delta$ given by a $n$-terms partial sum of series representation of $E\left(e^{\boldsymbol{\epsilon}}\right)$,

261 that is,

$262 \delta=\sum_{0}^{n^{E\left(\epsilon_{c}^{k}\right)}} \frac{}{k !}$.

263 Maximization of Lin's Concordance Correlation Coefficient (CCC) (Lin 1989) between

264 observed values and mean response $E_{g}(y \mid x)$ resulting using this form of $\delta$ sets criterion to

265 choose $n$.

\section{6 b.2) Huxley's formula of Simple Allometry}

267 A characterization of $w(x, \boldsymbol{p})$ as a power function $\beta x^{\alpha}$ has been traditionally referred as Huxley's

268 formula of simple allometry (Strauss \& Huxley, 1993). This model will be ahead epitomized by

269 a subscript $s$ as a mnemonic device for "simple". Equation (3) becomes

$270 y=w_{s}(x, \boldsymbol{p})+\epsilon$,

271 with $w_{S}(x, \boldsymbol{p})=\beta x^{\alpha}$ and $\epsilon$ assumed to be normally distributed with zero mean and variance $\sigma^{2}$,

272 that is, $\epsilon \sim \psi\left(0, \sigma^{2}\right)$. According to our notation convention equation (11) yields the mean

273 response function $E_{a s}(y \mid x)=\beta x^{\alpha}$.

274 Similarly, the logtransformation method produces the TAMA's regression model, that is,

$275 v=v_{s}(u, \pi)+\epsilon$

276 with

$277 v_{s}(u, \pi)=\ln \beta+\alpha u$ 
278 and $\epsilon$ as specified above. Equations (12) and (13) determine $E_{s}(v \mid u)=v_{s}(u, \pi)$. Accordingly,

279 back transformation of equation (12) to arithmetical space brings up a mean response $E_{g s}(y \mid x)$

280 given by

$281 E_{g s}(y \mid x)=\beta x^{\alpha} \delta$,

282 where $\delta$ stands for necessary CF.

283 It often occurs that even after contemplation of proper form for $\delta$ this TAMA's derived proxy for $284 w(x, \boldsymbol{p})$ produces biased projections of observed values of the response $y$. This means, that 285 complexity of Huxley's formula of simple allometry $w_{S}(x, \boldsymbol{p})$ becomes inappropriate to identify 286 the true $w(x, \boldsymbol{p})$ form (Gould, 1966; Huxley, 1932; Bervian et al. 2006; MacLeod, 2014;

287 Echavarria-Heras et al. 2019). From the settings of equation (1) it is reasonable assuming that 288 adapting complexity as it is needed to identify $w(x, \boldsymbol{p})$ could depend on MPCA forms.

289 Corresponding logtransformmed expression $v(u, \pi)$ is inferred to be a nonlinear function of 290 covariate $u$. This rears PLA as a likely device to acquire complexity for identification of MPCA 291 through geometrical space methods. We adopt the affine structure of a first order TSK fuzzy 292 model as a device for identification both MPCA or PLA alternates.

293 b.3) The TSK account of $w(x, p)$.

294 The general output of a first order TSK fuzzy model bears a fuzzy alternate to a statistical 295 mixture regression model (Cohn et al. 1997). It is then reasonable to assume that such an 296 structure could efficiently address the problem of identifying $w(x, \boldsymbol{p})$ expressed as a MPCA 297 model in arithmetical scale or its assumed PLA forms in geometrical space. The symbol $w_{\text {TSK }}$ $298(x, \boldsymbol{p})$ will stand for the TSK-MPCA surrogate for $w(x, \boldsymbol{p})$. Accordingly, adaptation of equation 299 (3) becomes

$300 y=w_{T S K}(x, \boldsymbol{p})+\epsilon_{T S K}$ 
301 with $\epsilon_{T S K}$ a $\psi$-distributed residual random variable with zero mean and variance expressed as a

302 function $\sigma_{T S K}^{2}(x)$ of $x$, that is, $\epsilon \sim \psi\left(0, \sigma_{T S K}^{2}(x)\right)$.

303 Denoting through the symbol $E_{a T S K}(y \mid x)$ the mean response function determined by equation

304 (15), we have

$305 E_{a T S K}(y \mid x)=w_{T S K}(x, \boldsymbol{p})$.

306 Since, the general output of a first order TSK fuzzy model can uniformly approximate any

307 continuous function to arbitrarily high precision (Ying, 1998; Zeng et al. 2000) then whatever

308 MPCA form $w(x, \boldsymbol{p})$ embraces, this can be accurately projected through a consistent identification

309 of $E_{a T S K}(y \mid x)$.

310 In turn, according to equation (4) the a TSK-PLA regression protocol becomes,

$311 v=v_{T S K}(u, \pi)+\epsilon_{T S K}$,

312 where according to equation $(5), v_{T S K}(u, \pi)=\log \left(w_{T S K}(x, \boldsymbol{p})\right)$ and $\epsilon_{T S K}$ as specified in equation 313 (15).

314 In turn, equation (17) yields $E_{T S K}(v \mid u)=v_{T S K}(u, \pi)$. Additionally, a back-transformation $e^{v}$ of 315 equation (17) sets

$316 y=\exp \left(v_{T S K}(u, \pi)\right) e^{\epsilon_{T S K}}$.

317 Then, corresponding mean response function in arithmetical space turns out to be

$318 E_{g T S K}(y \mid x)=\exp \left(v_{T S K}(u, \pi)\right) \delta$.

319 By assumption, we take $v_{T S K}(u, \pi)$ in the form given by equation (A14), that is,

320

$v_{T S K}(u, \pi)=\sum_{1}^{q} \vartheta^{i}(u) f^{i}(u)$ 
321 with $\vartheta^{i}(u)$ and $f^{i}(u)$ are one to one the firing strengths and consequent functions for $i=1,2, \ldots, q$.

322 Since, the domain $U$ of covariate is $R$, we can assume membership functions $\mu_{\Phi_{k}}(u)$ to have a

323 Gaussian form i.e.

$324 \mu_{\Phi_{k}}(u)=\exp \left\{-\frac{1}{2}\left[\left(\frac{u-\theta_{k}}{\lambda_{k}}\right)^{2}\right]\right\}$

325 being $\theta_{k}$ and $\lambda_{k}$ for $k=1,2, \ldots, q$, parameters. We also consider that consequent local models $f^{i}$

$326(u)$ have a description, that is,

$327 f^{i}(u)=\ln \beta_{i}+\alpha_{i} u$,

328 being $\alpha_{i}$ and $\ln \beta_{i}$ parameters. Readily, equations (20) through (22) entail a TSK-PLA

329 arrangement. As it will be clarified ahead a similar adaptation of equation (A14) stablishes the

330 TSK-MPCA form $w_{T S K}(x, \boldsymbol{p})$ in equation (15).

331 Identification of $v_{T S K}(u, \pi)$ as given by Eq. (20) through Eq.(22) is performed by means of the

332 Matlab function: main_fun_tsk_pla_model_fit.m. available from the supplemental files section.

333 Heterogeneity and reproducibility strength features of $v_{T S K}(u, \pi)$ can be interactively explored

334 through different characterizations of the clustering radius-training parameter $r_{a}$ as specified by

335 Eq. (B7) through Eq. (B9).

336 As described in Appendix A, acquiring $v_{T S K}(u, \pi)$ requires on first stage a fuzzy partition $L_{u}$ of

337 the input domain $U$ (cf. equation (A3)). Achieving this relies on Subtractive Clustering (SC after 338 this) technique to establish the value of the parameter $q$ (Castro et al. 2016; Chiu, 1994). A brief

339 description of the SC method is provided in Appendix B .This stage also sets the number of

340 inference rules $R^{i}$ specified by equation (A10) and concomitant number local models in $v_{\text {TSK }}$

$341(u, \pi)$. The SC step also produces estimates for the parameters $\theta_{k}$ and $\lambda_{k}$ in characterizing the 
342 membership functions $\mu_{\Phi_{k}}(u)$. Then, the normalized firing strength functions, $\vartheta^{i}(u)$ follows

343 from equations (A11) and (A12). A second step targets at characterization of the linear

344 consequents $f^{i}(u)$ as given by equation (22). This is achieved by replacing the identified factors

$345 \vartheta^{i}(u)$ and the assumed form of the consequents $f^{i}(u)$ into equation $(20)$ to characterize the

346 regression model of equation (17). Then, the parameters in the consequents $f^{i}(u)$ are estimated

347 by implementing a Recursive Least Squares (RLS) routine (Jang, Sun \& Mizutani, 1997; Wang

348 \& Mendel, 1992). This identification step could be also performed through a maximum

349 likelihood approach (Kalbfleisch, 1985). The whole identification algorithm is explained in

350 Appendix B. Codes are included in the supplemental files section.

351 b.4) Assessment of Reproducibility Strength of Models

352 The assessment of the reproducibility strengths of the proxies considered here will be primarily 353 carried away by analyzing values of Lin's concordance correlation coefficient, symbolized by 354 means of $\boldsymbol{\rho}_{\boldsymbol{C}}(\mathrm{Lin}, 1989)$. Agreement will be defined as poor whenever $\boldsymbol{\rho}_{\boldsymbol{C}}<0.90$, moderate for 355 $0.90 \leq \boldsymbol{\rho}_{C}<0.95$, good for $0.95 \leq \boldsymbol{\rho}_{\boldsymbol{C}}<0.99$, or excellent for $\boldsymbol{\rho}_{\boldsymbol{C}} \geq 0.99$ (MacBride, 2005).

Besides, CCC reproducibility will be estimated by means of model performance metrics, such as 357 the Coefficient of Determination (CD), Standard Error of Estimate (SEE), Mean Prediction Error 358 (MPE), Mean Percent Standard Error (MPSE) (Gupta et al. 1998; Hauduc et al. 2011; Zeng et al. 359 2017; Zeng \& Tang, 2011b). Related statistics are included in Appendix C. The Matlab and R 360 codes involved in both fuzzy inference and convention statistical task are provided in the 361 supplemental files section.

\section{Results}

363 a) Data 
364 Plots in Fig. 1 display the spread response-covariate in geometrical space for data sets included 365 in this examination. Panel (a) relates to the Echavarría-Heras et al. (2019) data. Panel (b) is for

366 Mascaro et al. (2014) data. Panel (c) shows Huxley's plot of chela mass vs body mass of fiddler

367 crabs (Uca pugnax) in log-log scale (Huxley, 1924; Huxley,1932). Panel (d) displays spread for

368 the De Robertis \& Williams (2008) data. Assessment of curvature will be performed for all data

369 sets by analyzing fitting results of the TSK-PLA and TSK-MPCA models. For easy of

370 presentation detailed results on a TAMA-TSK model comparison will only circumscribe to the

371 Echavarria-Heras et al. (2019) data.

372 b) Representation of the back-transformed TSK-PLA proxy as a MPCA formula

373 This section explains that assuming TSK-PLA implies a multiple parameter complex allometry

374 form in direct arithmetical scales. Indeed following Bervian et al. (2006), Echavarria-Heras et al.

375 (2019) proposed an extension of Huxley's formula of simple allometry $w_{s}(a, \boldsymbol{p})=\beta a^{\alpha}$ that

376 includes scaling parameters $\alpha$ and $\beta$ depending in the covariate, that is,

$377 y=\beta(x) x^{\alpha(x)}$

378 where $\beta(x)$ and $\alpha(x)$ are continuous functions and with $\beta(x)$ assumed to be positive. This sets

$379 w(x, \boldsymbol{p})=\beta(x) x^{\alpha(x)}$.

380 Thus, formally whenever the scaling functions $\beta(x)$ and $\alpha(x)$ are not simultaneously constant $w$ $381(x, \boldsymbol{p})$ as given by equation (24) entails MPCA.

382 We now demonstrate that the mean response function $E_{g T S K}(y \mid x)$ in arithmetical space derived 383 from a TSK-PLA arrangement implies MPCA in the form set by equation (24). For that drive, 384 we notice that replacing equation (22) into equation (20) and then rearranging leads to 385

$v_{T S K}(u, \pi)=\ln \beta_{T S K}(u)+\alpha_{T S K}(u) u$

where 
$387 \quad \beta_{T S K}(u)=e^{\sum_{1}^{q} \vartheta^{i}(u) \ln \beta_{i}}$

388 and

$389 \alpha_{T S K}(u)=\sum_{1}^{q} \vartheta^{i}(u) \alpha_{i}$

390 Thus, equation (17) takes on the equivalent representation,

$391 v=\ln \beta_{T S K}(u)+\alpha_{T S K}(u) u+\epsilon_{T S K}$.

392 The functions $\ln \beta_{T S K}(u)$ and $\alpha_{T S K}(u)$ above, suggest $u$-dependent forms of the parameters

$393 \ln \beta$ and $\alpha$ involved in the regression model of the TAMA approach. Then, under the assumption 394 of equation (22) a TSK-PLA arrangement interprets as generalization of the TAMA scheme 395 (Echavarria-Heras et al. 2019). Applying the back-transformation $e^{v}$ of equation (28) and 396 recalling equation (19) yields

$397 E_{g T S K}(y \mid x)=\beta_{T S K}(x) x^{\alpha_{T S K}^{(x)}} \delta$

398 This sets $\exp \left(v_{T S K}(u, \pi)\right)=\beta_{T S K}(x) x^{\alpha_{T S K}(x)}$. But, from equation (5) we have

$399 v_{T S K}(x, \boldsymbol{p})=\ln \left(w_{T S K}(x, \boldsymbol{p})\right)$ then $w(x, \boldsymbol{p})$ as identified by $v_{T S K}(x, \boldsymbol{p})$ admits the form specified by 400 equation (24).

401 c) Fitting results of the TAMA protocol: Zostera marina

402 For comparison aims, we present fitting results of the TAMA on the Echavarria-Heras et al. 403 (2019) data. Estimates for the allometric parameters $\alpha$ and $\beta$ derive from linear regression on 404 log-transformed data $(v, u)$ (cf. equation (12)). Table 1 summarizes the results of the analysis. 405 Corresponding, mean response $E_{s}(v \mid u)$ in geometrical scale is shown in Fig. 2a. Log406 transformation is a mechanism aimed to reduce variability of data (Feng et al. 2014).

407 Nevertheless, Fig. 2a still displays a significant dispersion of $v$ values about $E_{s}(v \mid u)$. Spread 
408 may lead on first sight to the impression that the distribution of $v$ around the mean response line $409 E_{s}(v \mid u)$ for small values of $u$ is fair. Agreeing with (Packard, 2017b), on the importance of 410 graphs in allometry, led to a careful revision of the spread which suggested curvature. Moreover, 411 the assessment of dispersion of residuals $\epsilon$ of equation (12) suggested lack of normality, as well 412 as, heteroscedasticity (Fig. 2b). Further, QQ plot shows heavier tails than expected for a normal 413 distribution (Fig. 2c). Indeed, an Anderson-Darling (1952) test to ascertain normality of $\epsilon$ 414 residuals produced a test statistics value of $A=310.848$ and a $p-$ value $<2.2 \mathrm{e}-16$. In turn a 415 Lilliefors (Kolmogorov-Smirnov-type) test, delivered $\mathrm{D}=0.1305$, as well as, a relatively small $416 \mathrm{p}$ - value $<2.2 \mathrm{e}-16$. Therefore, the hypothesis of normally distributed errors in the analysis 417 should be rejected since obtained p-values are extremely small $(<2.2 \mathrm{e}-16)$. What is more, a 418 lack of normality of $\epsilon$ errors in the linear regression analysis of equation (12) can be also 419 ascertained from the normal QQ plot shown in Fig. 2c. It can be perceived that the distribution of $420 \epsilon$ residuals exhibits heavier tails than those expected for a normal distribution.

421 Besides, a Breush-Pagan statistic (Breusch \& Pagan, 1997), provided a way to assess

422 heteroscedasticity of the $\epsilon$ residuals. In order to perform this test, the squared errors in the linear 423 model of equation (12) were assumed to depend linearly on the independent variable i.e.

$424 \epsilon(u)^{2}=b+d u+\zeta$

425 where $b$ and $d$ are parameters and $\zeta$ the error term. The null hypothesis is that the parameter $d$ in 426 equation (30) vanishes. Rejection of the null hypothesis not only corroborates heteroscedasticity 427 but also provides information on variability. The test statistics turned out to be $\mathrm{BP}=808.8119$ 428 with one degree of freedom with a p - value $(<2.2 \mathrm{e}-16)$, that is sufficiently small as to 429 provide strong evidence against homoscedasticity, while undoubtedly favoring 430 heteroscedasticity. Thus, the presently fitted straight line does not comply the essential 
431 assumptions of normality and homoscedasticity of the analysis. Therefore, the TAMA protocol

432 turned out unsuited for analyzing the allometric relationship in the Echavarria-Heras et al. (2019)

433 data. Consequently, characterization of the general function $w(a, \boldsymbol{\theta})$ entailed by Huxley's model

434 of simple allometry (cf. Eq. 11) does not fit required complexity. Thus data spread shown in Fig.

$4352 \mathrm{a}$, submits curvature in geometrical space. We now turn to explore the capabilities of the TSK-

436 PLA construct to identify this pattern.

437 d) Fitting results of the TSK-PLA assembly: Zostera marina

438 d.1) Identification of firing strength factors $\vartheta^{i}(u)$

439 In order to identify the required firing strength factors $\boldsymbol{\vartheta}^{i}(\boldsymbol{u})$ for $i=1,2, \ldots, q$. We executed the

440 main_fun_tsk_pla_model_fit.m code on log-transformed values $(v, u)$ from the Echavarria-

441 Heras et al. (2018a) data set. This try assumed membership functions $\mu_{\Phi_{k}}(u)$ having a form

442 given by equation (21) for $k=1,2, \ldots, q$. Setting $r_{a}=0.47$ of returned a value of $q=2$. Then, we

443 have to consider two membership functions characterizing the fuzzy partition of imput space $U$.

444 Moreover, in compliance with equation (A12) normalized firing strength factors $\vartheta^{1}(u)$ and $\vartheta^{2}$

$445(u)$ turn out to be

$446 \vartheta^{1}(u)=\frac{1}{1+\exp \left\{-\frac{1}{2}\left[\left(\frac{u-\theta_{2}}{\lambda_{2}}\right)^{2}-\left(\frac{u-\theta_{1}}{\lambda_{1}}\right)^{2}\right]\right\}}$

$447 \vartheta^{2}(u)=\frac{\exp \left\{-\frac{1}{2}\left[\left(\frac{u-\theta_{2}}{\lambda_{2}}\right)^{2}-\left(\frac{u-\theta_{1}}{\lambda_{1}}\right)^{2}\right]\right\}}{1+\exp \left\{-\frac{1}{2}\left[\left(\frac{u-\theta_{2}}{\lambda_{2}}\right)^{2}-\left(\frac{u-\theta_{1}}{\lambda_{1}}\right)^{2}\right]\right\}}$

448 Plots of the estimated membership functions $\mu_{\Phi_{1}}(u)$ and $\mu_{\Phi_{2}}(u)$ and normalized firing strength

449 factors $\vartheta^{1}(u)$ and $\vartheta^{2}(u)$ appear in Fig. 3a and Fig $3 b$ respectively. We observe that agreeing 450 curves intersect at a point $u_{b}=3.998$.

451 d.2) Identification of consequent functions $\boldsymbol{f}^{\mathbf{i}}(\boldsymbol{u})$ 
452 A second step in the procedure to get $v_{T S K}(u, \pi)$ concerns acquiring the consequent functions $f^{\mathrm{i}}$

453 ( $u$ ) in equation (22). Since, for this data, we obtained $q=2$, the code ought to establish

454 consequent functions $f^{1}(u)$ and $f^{2}(u)$, each one assumed to be linear, that is,

$455 f^{1}(u)=\ln \beta_{1}+\alpha_{1} u$

456 and

$457 f^{2}(u)=\ln \beta_{2}+\alpha_{2} u$.

458 With the aim of assessing heteroscedasticity, we replaced the forms of $\vartheta^{1}(u)$ and $\vartheta^{2}(u)$

459 identified by SC technique in regression equation (17). In turn the involved consequent functions

$460 f^{1}(u)$ and $f^{2}(u)$ were assumed to have both the form given by equations (33) and (34)

461 correspondingly. Then, we assumed the involved $\epsilon_{T S K}$ residuals to be normally distributed with

462 zero mean, but with a standard deviation set as a function $\sigma_{T S K}(u)$ of the covariate $u$, Namely

$463 \sigma_{T S K}(u)=\log (\sigma+k u)$,

464 where $\sigma$ and $k$ are parameters to be estimated and such that $\sigma+k u>1$. Table 2 presents

465 maximum-likelihood parameter estimates and associated uncertainties for the related fit. We can

466 ascertain a highly significant fit, since, in all cases the standard error is extremely small, this

467 mainly explained by the large amount of data in the analysis. To judge heteroscedasticity of

468 residuals we study the confidence interval of parameter $k$. This parameter determines the change

469 in residual variability per unit change in $u$. It turns out that figures in Table 2 show that

470 confidence interval of parameter $k$ does not include a zero value. Therefore, we may conclude

471 that with high probability the variability of the residuals changes as $u$ changes.

472 Meanwhile, setting $k=0$ in equation (35) allowed consideration of a parallel maximum

473 likelihood fit of homoscedastic case of the TSK regression model of equation (17). Table 3 
474 provides fitting results. Model performance metrics allow assessment of the fits of the

475 heteroscedastic and homoscedastic versions of the TSK -PLA protocol. Accordingly, we can

476 assert that the heteroscedastic model stands a better fit than its homoscedastic counterpart.

477 Particularly, in the heteroscedastic case we have a negative log-likelihood value of - $\log L$

$478=6304.60$, which turns out to be notably smaller than $-\log L=8111.49$ obtained for the

479 homoscedastic model. These figures bear a notable difference that backs the selection of the

480 heteroscedastic model. This difference in fit quality favoring the heteroscedastic model is mainly

481 due to the fact that the latter models the pattern of variation of the errors in a more reliable way.

482 Plots of identified consequents appear in Fig. 3c, component products $\vartheta^{1}(u) f^{1}(u)$ and $\vartheta^{2}(u) f^{2}($

$483 u$ ) appear in Fig. 3d. As it occurs for the membership functions and firing strength factors for

484 this data the component products also intersect at value of $u_{b}=3.98$. This estimate of $u_{b}$ is

485 consistent with value previously reported by Echavarria-Heras et al. (2019) for this data and

486 deriving from conventional maximum likelihood biphasic regression. Fig. 4a displays dispersion

487 about resulting mean response function $v_{T S K}(u, \pi)$. Fig. $4 \mathrm{~b}$ shows residual scattering about the

488 zero line. Region bounded by red lines determine (95\%) confidence intervals. Fig. $4 \mathrm{c}$ shows

489 corresponding QQ plot.

\section{0 d.3) Comparison TAMA vs TSK-PLA}

491 Compared with corresponding fitting results for the TAMA protocol (Fig. 2a) we can verify that

492 plots in Fig. 4 show fairer residual spread patterns. Nevertheless, the QQ plot in Fig. 4c, still

493 suggest deviation of $\epsilon_{T S K}$ residuals from a normal distribution pattern. Table 4 allows further

494 comparison of performances of the TAMA and TSK proxies. This undoubtedly favor selection of

495 the TSK scheme. Therefore, opposed to the linear model $v_{s}(u, \pi)$, the affine characterization of

496 variability granted by $v_{T S K}(u, \pi)$ can better refer to inherent non-log linear allometry for the 
497 Echavarria-Heras et al. (2019) data set. Certainly, the point $u_{b}=3.98$ shown in Fig. $3 \mathrm{~b}$ can be 498 interpreted as a point separating two phases describing the variation pattern of the $\log$ 499 transformed response $v$. One for small leaves valid over the region $u<u_{c}$ and another for large 500 leaves over $u \geq u_{b}$. The form of the component products $\vartheta^{i}(u) f^{i}(u)$ shows that while $u$ drifts 501 away from $u_{b}$ taking smaller and smaller values the closer the TSK output $v_{T S K}(u, \pi)$ will be to 502 the component product $\vartheta^{1}(u) f^{1}(u)$. Conversely, the larger the distance between $u$ and $u_{b}$ for 503 leaves in the large phase $u \geq u_{b}$ the closer $v_{T S K}(u, \pi)$ will be to $\vartheta^{2}(u) f^{2}(u)$. Relating to $E_{S}(v \mid u)$ 504 shown in Fig. 2a, we can assess from Table 4 and Fig. 4a that the reproducibility strength of $505 E_{T S K}(v \mid u)$ is higher. Besides compared with Fig. 2b, the plot in Fig. 4b shows that distribution of 506 residuals about the zero line for the TSK fit improved. Also compared to Fig. 2c, normal QQ plot 507 in Fig. 4c shows a larger plateau where $\epsilon_{T S K}$ residuals track a normal distribution pattern.

508 Nevertheless, application of an Anderson-Darling (1952) test to the residuals of regression 509 equation (17) resulted in $A D=370.17$. This associates a p-value $<2.2 \times 10^{-16}$, that provides 510 evidence against a normality assumption for the $\epsilon_{T S K}$ residuals. This is, in agreement with the 511 observation that he normal Q-Q plot shown in Fig. 4c showing heavier tails than those expected 512 for a normal distribution. It is worth pointing out that the break point $u_{b}$ identified by the fuzzy

513 proxy $v_{T S K}(u, \pi)$ coincides with corresponding value obtained by Echavarria-Heras et al. (2019)

514 using conventional biphasic regression methods.

515 Correspondingly, Fig. 5a displays the plot of the estimated form of the mean response function $516 E_{g T S K}(x \mid y)$ of equation (19). Since, residuals $\epsilon_{T S K}$ are not normally distributed, equation (10) 517 provided CF form. Fig. 5b allows a visual assessment of the extent of biased projections in 518 arithmetical scale tied to the TAMA surrogate $E_{g s}(x \mid y)$ calculated with Duan's form of $\delta$. 
519 Compared with spread deriving from the TSK model, TAMA's bias is significant. Besides, Table

5204 allows assessment of differences in associated predictive strengths. All indices favor the TSK -

521 PLA scheme. As suggested by perceptible bias shown in Fig. 5b, CCC value for TAMA's

522 projections point to poor reproducibility of observed values. Besides, relevance of accounting for

523 curvature, this assessment highlights on the importance of choosing a proper form of $\delta$ for

524 assuring consistency or retransformation results.

525 d.4) Asymptotic analysis of TSK-PLA assembly

526 In this section we explain that adoption of a TSK-PLA approach allows exploration of

527 asymptotic behavior of the allometric mean response function in arithmetical space. After

528 replacing $f^{1}(u)$ and $f^{2}(u)$ as given by equations (33) and (34) in equation (20) direct algebraic

529 manipulation yields

530

$v_{T S K}(u, \pi)=\ln \left(\beta_{1}^{\left(1-\vartheta^{2}(u)\right)} \beta_{2}^{\vartheta^{2}(u)}\right)+\left(\alpha_{1}\left(1-\vartheta^{2}(u)\right)+\alpha_{2} \vartheta^{2}(u)\right) u$

531 Similarly, it can be directly verified that firing strengths $\vartheta^{1}(u)$ and $\vartheta^{2}(u)$ as given by equations

532 (31) and (32) can be also expressed in the form

$533 \vartheta^{1}(u)=\frac{1}{1+e^{\tau(u, \theta, \lambda)}}$

534 and

$535 \vartheta^{2}(u)=\frac{e^{\tau(u, \boldsymbol{\theta}, \lambda)}}{1+e^{\tau(u, \boldsymbol{\theta}, \lambda)}}$,

536 where

$537 \tau(u, \boldsymbol{\theta}, \boldsymbol{\lambda})=\psi(\boldsymbol{\lambda}) \phi(u, \boldsymbol{\theta}, \boldsymbol{\lambda})+\xi(\boldsymbol{\theta}, \boldsymbol{\lambda})$,

$538 \psi(\lambda)=\frac{\left(\lambda_{2}^{2}-\lambda_{1}^{2}\right)}{2\left(\lambda_{1} \lambda_{2}\right)^{2}}$,

$539 \phi\left((u, \boldsymbol{\theta}, \lambda)=\left[u+\left(\frac{\lambda_{2}{ }^{2} \theta_{1}-\lambda_{1}{ }^{2} \theta_{2}}{\lambda_{1}{ }^{2}-\lambda_{2}{ }^{2}}\right)\right]^{2}\right.$ 
$540 \xi(\boldsymbol{\theta}, \lambda)=\frac{\left(\lambda_{2}^{2} \theta_{1}-\lambda_{1}^{2} \theta_{2}\right)^{2}-\left(\lambda_{1}^{2}-\lambda_{2}^{2}\right)\left[\left(\lambda_{1} \theta_{2}\right)^{2}-\left(\lambda_{2} \theta_{1}\right)^{2}\right]}{2\left(\lambda_{1}^{2}-\lambda_{2}^{2}\right)\left(\lambda_{1} \lambda_{2}\right)^{2}}$.

541 with $\boldsymbol{\theta}, \boldsymbol{\lambda}$ standing for parameter vectors $\left(\theta_{1}, \theta_{2}\right)$ and $\left(\lambda_{1}, \lambda_{2}\right)$ one to one. We can then ascertain

542 that $\phi(u, \boldsymbol{\theta}, \lambda)$ remains positive for all values of $u$. Also, the term $\xi(\boldsymbol{\theta}, \lambda)$, does not depend on $u$.

543 Consequently, whenever the factor $\psi(\boldsymbol{\lambda})$ in equation (40) is positive, $\tau(u, \boldsymbol{\theta}, \boldsymbol{\lambda})$ will approach

544 infinity as $u$ approaches infinity. Then, equation (37) implies $\vartheta^{1}(u)$ asymptotically vanishing as

$545 u$ approaches infinity. Reversely, whenever $\psi(\lambda)$ is negative, the firing strength factor $\vartheta^{1}(u)$ will

546 asymptotically approach one as $u$ approaches infinity. For the Echavarria-Heras et al. (2019) data

547 set we obtained $\psi(\lambda)=0.2756$, then we must have

$548 \lim _{u \rightarrow \infty} \vartheta^{1}(u)=0$

549 and since equation (A13) implies $\vartheta^{2}(u)=1-\vartheta^{1}(u)$, we also have

$550 \lim _{u \rightarrow \infty} \vartheta^{2}(u)=1$.

551 Agreeing with equations (19-20) back-transformation $e^{v}$ produces

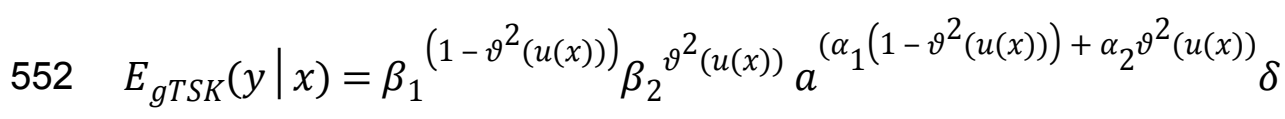

553 We denote by means of the symbol, $E_{g T S K}^{\infty}(y \mid x)$ the limit of $E_{g T S K}(y \mid x)$ as given above as $x$

554 approaches infinity, that is,

$555 E_{g T S K}^{\infty}(y \mid x) \underset{a \rightarrow \infty}{=\lim _{g T S K}} E_{g \mid x) .}$

556 Then, equation (31), (32) and (44) through (46) imply

$557 E_{g T S K}^{\infty}(y \mid x)=\beta_{2} x^{\alpha} \delta$

558 Then, the asymptotic mode $E_{g T S K}^{\infty}(y \mid x)$ identified for the Echavarria-Heras et al. (2019) data

559 set, is attains a form like Huxley's formula of simple allometry $w_{s}(x, \theta)$. Estimated parameters 
560 are $\alpha_{2}=1.1126$ and $\beta_{2}=7.8398 \times 10^{-6}$. Fig. 5c displays observed leaf biomass values $y$ and

561 their projections through the $E_{g T S K}^{\infty}(y \mid x)$ proxy. We can learn of a remarkable correspondence

562 between the power function $w_{s}(x, \boldsymbol{\theta})=\beta x^{\alpha}$ of equation (11) fitted by direct nonlinear regression

563 methods and the asymptotic mean response $E_{g T S K}^{\infty}(y \mid x)$. Besides as established by equation (45)

564 we can directly asses from Fig. 5c that for sufficiently large values of $x$ in the Echavarría-Heras

565 et al. (2019) data set, the mean response $E_{g T S K}(y \mid x)$ behaves as the power function $E_{g T S K}^{\infty}(y \mid x)$.

566 Moreover, the order relationship $u \geq u_{b}$ holds for about $86 \%$ of analyzed data. This explains

567 why corresponding phase of the TSK output can be considered dominant. This by the way

568 elucidates the apparent benefit of fitting Huxley's formula of simple allometry by means of

569 nonlinear regression model directly in arithmetical scale for the considered data. Indeed, such a

570 fitting could deliver reasonable model adequacy results. But, as the present results show direct

571 nonlinear examination based on Huxley's formula of simple allometry will fail to detect the

572 different allometrical phases conforming the real variation pattern in the data. Then, as we have

573 elaborated a log transformation step followed by nonlinear model identification in geometrical

574 space could overcome the reproducibility deficiency of the TAMA approach.

575 e) Fitting results of the TSK-PLA assembly: Metrosideros polymorpha

576 For trying the main_fun_tsk_pla_model_fit.m function on the Mascaro et al (2011) data we set $577 r_{a}=0.80$. This returned $q=2$, heterogeneity. Fig. 6 displays the plots of membership

578 functions $\mu_{\Phi_{i}}(u)$, firing strength factors $\vartheta^{i}(u)$, consequent linear segments $f^{i}(u)$ and component

579 products $\vartheta^{i}(u) f^{i}(u)$ identified by the fit of the TSK fuzzy model to the Mascaro et al. (2011)

580 data set. Membership functions are shown in Fig 6a. Fit suggests heterogeneity determined by a

581 break point $u_{b}=1.575$ as shown in Fig. $6 \mathrm{~b}$ displaying firing strength factors. This estimate of 
$582 u_{b}$ is consistent with value previously reported by Echavarria-Heras et al. (2019) for this data and

583 deriving from conventional maximum likelihood biphasic regression. Break point suggest a

584 growth phase $0<u \leq u_{b}$ and a complementary $u>u_{b}$. We can interpreted these regions as

585 dominance realms for the component product functions $\vartheta^{1}(u) f^{1}(u)$ and $\vartheta^{2}(u) f^{2}(u)$ one to

586 one (Fig. 6c and Fig. 6d). Correspondingly, Fig. 7a shows spread about mean response function

587 regions in geometrical space matching identified phases. Moreover, residual plot in Fig. 7b

588 displays a fair distribution about the zero line. And, normal QQ-plot in Fig. 7c shows a large

589 plateau where residuals track a normal distribution pattern. We can also ascertain from goodness

590 of fit statistics in Table 5, that compared to the linear regression scheme of the TAMA protocol,

591 the affine modeling approach composing the TSK-PLA scheme entails consistent identification

592 of curvature in geometrical space.

593 f) Identification of the TSK-PLA proxy: Uca pugnax

594 Huxley conceived a breakpoint in the log-log plot of chela mass vs body mass of fiddler crabs

595 (Uca pugnax). (Huxley 1924; Huxley 1932). Huxley situated this point between the 15th and

596 16th observations and assumed it meant a to a sudden change in relative growth of the chela

597 approximately when crabs reach sexual maturity. Examination of Huxley's data by Packard

598 (2012a) implied such a break point to be only putative and in Packard's own interpretation,

599 perhaps explained by the fact that Huxley could have been misled by the effects of the log

600 transformation itself, along with the format of graphical display that might have exaggerated the

601 slopes of segments before and after the change point. In order to test the performance of the

602 TSK-PLA protocol in analyzing Huxley's Uca pugnax data, we took averages of both body

603 mass and chela mass form Table 1 in Huxley's report (Huxley, 1932). Concurrent log

604 transformed values appear in Fig.1c. For easy of presentation a break point as conceived by 
605 Huxley's will be denoted here through the symbol $u_{b H}$. One substantial advantage of the fuzzy

606 logical approach over conventional probabilistic approaches is that the former facilitates

607 knowledge based modeling. In order to incorporate previous knowledge, we addressed Huxley's

608 assertion of biphasic allometry in Uca pugnax. Then, we examined heterogeneity patterns

609 predicted by the TSK-PLA system for different values of clustering radius $r_{a}$. Particularly,

610 setting $r_{a}=0.8$ returned $q=2$, arranging biphasic allometry. Acquired firing strengths appear

611 in Fig.8a, exhibiting a break point at $u_{b}=5.831$. Fig. $8 \mathrm{~b}$ display consequent linear functions

612 with estimated slopes $\alpha_{1}=1.2676$ and $\alpha_{2}=1.4708$ one to one respectively. In the settings of

613 performed TSK-PLA analysis these correspond to exponents characterizing allometric phases as

614 conceived in Huxley's original theoretical standpoint. Correspondingly, Fig. 8c portrays

615 consequent component products $\vartheta^{1}(u) f^{1}(u)$ and $\vartheta^{2}(u) f^{2}(u)$. Similarly, Fig.8d shows spread

616 about mean response $v_{T S K}(u, \pi)$ including placement of $u_{b}$ in a display in compliance with that

617 in Fig. 3 in Huxley (1932). We can be aware that location of $u_{b}$ is shifted back relative to $u_{b H}$.

618 Fig.8e displays placement of $u_{b}$ and spread about $v_{T S K}(u, \pi)$ in the original scale of data (cf. Fig.

619 1c). But instead, we may integrate previous knowledge by considering for that the break point

$620 u_{b H}$ actually exists. Then, we can search among different values of $r_{a}$, the one for what the TSK-

621 PLA arrangement compromises a break point $u_{b}$ placed as $u_{b H}$ and a maximum reproducibility

622 strength of interpolation by $v_{T S K}(u, \pi)$. Accordingly, setting $r_{a}=0.2$ brought about $q=7 \mathrm{sub}$

623 models, inducing a maximum reproducibility strength of interpolation function $v_{T S K}(u, \pi)$ and

624 where $u_{b l}$, one of six detected break points is placing as $u_{b H}$. (Fig.8e and Table 6). Interestingly,

625 visual examination of plot showing $v_{T S K}(u, \pi)$ suggests a pattern accommodating two linear

626 segments that alternate about $u_{b I}$. Moreover, using the interpolation points $\left(u, v_{T S K}(u, \pi)\right)$ we fit 
627 two linear segments of slopes $\alpha_{1 \mathrm{I}}=1.626$ and $\alpha_{2 \mathrm{I}}=1.274$ before and after $u_{b I}$ one to one

628 (Fig. 8f). Since $u_{b I}$. can be taken as a proxy for $u_{b H}$ the TSK-PLA interpolation mode could

629 suggest Huxley's reasoning of biphasic allometry in in Uca pugnax as consistent. In the

630 meantime acquired $q=7$, interpolation confirms the outstanding capabilities of the TSK-PLA

631 device to approximate the dynamics of the logtransformmed allometric response. This can be

632 better ascertained from Fig. 9a through Fig. 9c presenting spread about the high order

633 interpolation function $v_{T S K}(u, \pi)$, as well as, concomitant residual and QQ plots in conforming

634 order. Moreover, Fig. 9d through Fig. 9f allow visual comparison of parallel results by a

635 TAMA's fit. Additionally, Table 6 compares model performance metrics for the TSK-PLA

636 interpolation and TAMA's output fits. We can ascertain that the TSK-PLA interpolation stands a

637 better fit. In any event, the non-probabilistic interpretation of uncertainty backing the TSK-PLA

638 approach seems to advocate biphasic heterogeneity in geometrical space for Huxley's $U c a$

639 pugnax data.

640 g) Fitting results of the TSK- PLA assembly: Gadus chalcogrammu

641 A fit of the TSK-PLA protocol to Gadus chalcogrammu data reported in De Robertis \&

642 Williams (2008), can exhibit reliability of this paradigm in further way. Visual examination of

643 spread in geometrical space may suggest curvature. But, setting $r_{a}=0.5$ led to highest

644 reproducibility of $v_{T S K}(u, \pi)$ characterized in a linear form. Indeed, plots in Fig. 10 show that

645 identification of the TSK-PLA model for this data, produced only one membership function $\mu_{\Phi_{1}}($

$646 u)$ (Fig. 10a) This corresponds to a firing strength $\vartheta^{1}(u)$ set to one (Fig. 10b), and a conforming

647 single TAMA's form linear consequent $f^{1}(u)$ (Fig. 10c). This matched the single linear

648 component product function shown in panel (Fig. 10d). As a result, no heterogeneity as 
649 determined by breaking points $u_{b}$ was detected for this data. Consequently, the TSK arrangement

650 suggests a fit equivalent to the TAMA approach. Moreover, spread abut mean response, residual

651 and Normal QQ-plots for a TAMA fit performed in this data (Fig. 11a through Fig. 11c

652 respectively) seem to faithfully agree to corresponding plots (Fig. 11d, through Fig. 11f)

653 associating to the TSK-PLA fit. In turn model performance metrics in Table 7 corroborate these

654 alternate fits as equivalent. Thus performance of the TSK-PLA assembly confirms it seemingly

655 adapts to required complexity. This supports judgement on this paradigm being considered as a

656 generalized tool for allometric examination in geometrical space.

657 h) Assembly of the TSK- MPCA proxy

658 We assume that $w(x, \boldsymbol{p})$ as intended for MPCA can be modeled by $w_{T S K}(x, \boldsymbol{p})$ as expressed by

659 means of equation (A14), in arithmetical space, namely

$660 w_{T S K}(x, \boldsymbol{p})=\sum_{1}^{q} \vartheta^{i}(x) f^{i}(x)$

661 with firing strengths $\vartheta^{i}(x)$ given by

$662 \vartheta^{i}(x)=\frac{\mu_{\Phi_{i}}(x)}{\sum_{1}^{q} \mu_{\Phi_{k}}(x)}$

663 being $\mu_{\Phi_{i}}(x)$ for $i=1,2 \ldots \ldots . . q$ the involved membership functions. We also undertake that both

$664 w(x, \boldsymbol{p})$ and $x$ remain positive, and that

$665 \lim _{x \rightarrow 0^{+}} w(x, \boldsymbol{p})=0$

666 This sets the imput space $X$ to be $R^{+}$. We then contemplate that membership functions can be

667 expressed through a composite log normal form that satisfies the constrain by equation (50),

668 namely

$669 \mu_{\Phi_{i}}(x)=c\left(e^{h_{i}(x)}-1\right)$ 
670 where $c=(1-e)^{-1}$ and

$671 \quad h_{i}(a)=e^{\left\{-\frac{1}{2}\left[\left(\frac{\ln a-\theta_{i}}{\lambda_{i}}\right)^{2}\right]\right\}}$

672 with $\theta_{i}$ and $\lambda_{i}$ for $i=1,2, \ldots ., q$ parameters. Correspondingly we consider the consequents $f^{i}(x)$

673 to be linear functions, that is,

$674 f^{i}(x)=c_{i 1}+c_{i 2} x$

675 It is worth recalling that equation (15) provides the form of linked regression protocol.

676 Identification of $w_{T S K}(x, \boldsymbol{p})$ as given by Eq. (48) through Eq. (53) from data pairs $(y, x)$ in direct

677 scale is performed by means of the Matlab function main_fun_tsk_mpca_model_fit.m supplied

678 in the supplemental files section. Heterogeneity and reproducibility strength features of $w_{T S K}$

$679(x, \boldsymbol{p})$ can be explored in an interactive way through different characterizations of the clustering 680 radius parameter $r_{a}$ as specified by Eq. (B7) through Eq. (B9).

681 i) Identification of the TSK-MPCA proxy: Zostera marina

682 For the Echavarria-Heras et al. (2019) data a try of the main_fun_tsk_mpca_model_fit.m code

683 setting $r_{a}=0.5416$ returned $q=2$ for a biphasic mode and a maximum reproducibility of $w_{T S K}$

$684(x, \boldsymbol{p})$. Fig. 12a displays acquired firing strength functions. The estimated break point was $x_{b}$

$685=49.632$ consistent with the retransformed value of $u_{b}=3.9$ for this data set. This means that

686 variability of the response $y$ indeed conforms to a MPCA pattern in the direct scale of data.

687 Corresponding spread about fitted mean response function $E_{a T S K}(y \mid x)$ appear in Fig. 12b. This

688 plot allows comparison to its counterpart $E_{g T S K}(y \mid x)$ produced by retransformation of mean

689 function $v_{T S K}(u, \pi)$ to arithmetical space. We can be aware of remarkable correspondence

690 through $x$ values. This validates adequacy of a TSK-PLA analysis for this data. Fig. 12c and Fig. 
691 12d show residual spread and QQ-plot for the TSK-MPCA fit. Fig. 12e and Fig. 12f show

692 corresponding plots for retransformed TSK-PLA fit. Besides Table 8 allows assessment of

693 addressed proxies through model performance metrics. This could endure a judgement that

694 concurrent MPCA pattern in arithmetical space can be consistently characterized by

695 retransformation of PLA results.

\section{6 j) Identification of the TSK-MPCA proxy: Metrosideros polymorpha}

697 Correspondingly, taking as previous knowledge a manifestation of biphasic allometry as detected

698 by the TSK-PLA scheme, for the Mascaro et al. (2011), we examined the possibility of the TSK-

699 MPCA arrangement identifying a similar pattern in direct scales. Indeed, by setting $r_{a}=0.855$

700 the main_fun_tsk_mpca_model_fit.m function returned $q=2$ for a biphasic mode and a $w_{T S K}$

$701(x, \boldsymbol{p})$ of reliable reproducibility. Identified firing strengths, display in Fig. 13a. Again analysis in

702 direct scale detected by the TSK-MPCA approach corroborates the consistency break point

703 allometry for this data. We can learn of a break point estimated at $a_{b}=8.8662$. This estimate is

704 consistent with resulting from a two linear segment mixture regression model performed by

705 present authors. Spreads about fitted mean functions shown in Fig. 13b reveal remarkable

706 correspondence of projections by $E_{a T S K}(y \mid x)$ and $E_{g T S K}(y \mid x)$. This can be stressed by

707 performance metrics in Table 9. In turn this demonstrates adequacy of a TSK-PLA approach for

708 the analysis of this data. Fig. 13c and Fig. 13d display residual and QQ plots for TSK-MPCA fit

709 to Metrosideros polymorpha. Equivalent plots associating to the retransformed TSK- PLA are

710 displayed in Fig. 13e and Fig. 13f, correspondingly. Exploring interpolation capabilities of the

711 TSK-MPCA for this data led to considering an alternate clustering radius set at a value $r_{a}=0.52$

712 . Resulting heterogeneity index was $q=3$ that resulted in good model assessment metrics (Table

7139 ) and a break point placed at $x_{b}=4.832$. This is in agreement with retransformed TSK-PLA 
714 estimation for this data. Nevertheless, forcing an interpolation mode of the TSK-MPCA to

715 achieve a break point placed in agreement with previous estimation brings about complexity that

716 renders biological interpretation difficult.

\section{7 k) Identification of the TSK-MPCA proxy: Uca pugnax}

718 Firing strengths, of a $r_{a}=0.668, q=2$, TSK-MPCA fit to Huxley's Uca pugnax data set are

719 displayed in Fig. 14a. We can be aware of heterogeneity as corresponds to dominance of sub

720 models before and after the break point placed at $x_{b}=340.7$, matching $\exp \left(u_{b}\right)$ with $u_{b}=5.83$

721 the break point determined by a TSK-PLA fit to this data. Fig. 14b show spread about

722 resulting mean function $E_{a T S K}(y \mid x)$ and compares to $E_{g T S K}(y \mid x)$ gotten by retransformation of

723 fitted TSK-PLA. Plot suggest equivalent reproducibility strengths. Nevertheless as it can be

724 made certain by model performance metrics in Table10 the $E_{g T S K}(y \mid x)$ proxy entails relatively

725 higher reliability. Fig. 14c and Fig. 14d one to one show residual and QQ plots corresponding to

726 the TSK-MPCA fit. Similarly, residual and QQ plots for the back-transformed TSK-PLA fit

727 appear in Fig. 14e and Fig. 14f. Table 10 also includes performance metrics for $E_{g T S K}(y \mid x)$

728 gotten by retransforming the output of the $r_{a}=0.2$ and $q=7$, fit of the TSK-PLA. We can assert

729 that resulting interpolation $E_{g T S K}(y \mid x)$ yields a relatively better fit. Therefore, results of the

730 retransformed form of a TSK-PLA approach entails consistent results in direct scales. In other

731 words, logtransformation based procedures do not lead to biased results for this data. But above

732 all, results of a TSK-MPCA fit could provide a clue clearing an apparent misinterpretation of

733 Huxley about existence of a break point in his analysis of Uca pugnax data. Indeed, as stated

734 above we have $u_{b}=\ln \left(a_{b}\right)$. This implies $u_{b}$ being the image of $a_{b}$ under logtransformation.

735 Then, claiming existence of $u_{b}$ attributable to distortion set this device itself is inappropriate.

736 Agreeing with Packard (2012a), we have no doubt that conventional statistical methods do not 
737 put up with existence of $u_{b}$ as detected by the present fuzzy inference system. But, this fact

738 cannot be exhibited to question fuzzy methods. These relying in non-probabilistic approaches

739 have provided reliable interpretation of uncertainty as it can be inferred by fuzzy approach

740 solutions to many problems of identification and control of nonlinear systems.

\section{I) Identification of the TSK-MPCA proxy: Gadus chalcogrammu}

742 When we assessed the performance of the TSK-MPCA device on the De Robertis and Williams

743 (2008) data we found results consistent to the TSK-PLA fit reported above. Indeed, a TSK-

744 MPCA analysis based on $r_{a}=0.50$ for this data returned $q=1$, for a single membership

745 function. This consequently associates to a single firing strength $\vartheta^{1}(x)=1$. As a result, we have

746 to contemplate a single component product of a linear form in equation (48). No break point

747 composed heterogeneity in direct scales can be verified for this data. Moreover, implied linear

748 form of Eq. (48) does not fit required complexity in direct scales. Nevertheless, previous

749 knowledge on consistency of corresponding TSK-PLA fit suggest using the interpolation

750 features of TSK-MPCA to grant adequacy. For this empirical aim, for instance taking the

751 clustering parameter $r_{a}=0.22$ in equation (B7) we can manage to obtain an heterogeneity index

752 of $q=3$. This entails three sub models composing Eq. (48). Fig. 15a through Fig. 15c display

753 spread about resulting form of interpolation mean response function $E_{\text {aTSK }}(y \mid x)$, as well as,

754 residual and normal QQ plots in that order. Figure 15a also allows visual appraisal of a better

755 reproducibility by $E_{g T S K}(y \mid x)$. Figure $15 \mathrm{~d}$ presents spread about $E_{\text {as }}(y \mid x)$ and compares to

$756 E_{g T S K}(y \mid x)$. We can observe that both proxies entitle similar reproducibilities. Figure 15e and

757 Figure $15 \mathrm{f}$ present residual spread and QQ plot accompanying $E_{a s}(y \mid x)$. Table 11 compares

758 reproducibility metrics for the $E_{a T S K}(y \mid x), E_{g T S K}(y \mid x)$ and $E_{a s}(y \mid x)$ proxies for this data. Again 
759 confrontation of model performance metrics shows that retransformation of the TSK- PLA

760 output stands reliable results in direct scales.

\section{Discussion}

762 A logarithmic transformation in allometry is often vindicated as a natural way to lodge a

763 variation pattern resulting from multiplicative growth in plants and animals. Indeed, Gingerich

764 (2000) and Kerkhoff \& Enquist (2009) state that a number of biological processes, (i.e., growth,

765 reproduction, metabolism and perception), are essentially multiplicative and are therefore prone

766 to fit in better to a geometric error model. Beyond biological arguments supporting the

767 traditional approach, Kerchhoff \& Enquist (2009) underline that fitting models to log-

768 transformed data is seamlessly adequate, since taking into account proportional rather than

769 absolute variation is more significant. Therefore, from this standpoint, the fact that log-

770 transformation places numbers into a geometric domain could bestow advantages beyond a

771 purely statistical convenience. Nevertheless, there are remarks that a logtransformation approach

772 procedure produces biased results, and that direct nonlinear regression methods in arithmetical

773 scale, should be preferred in parameter identification tasks (e.g. Packard, 2013; Packard, 2009;

774 Packard \& Birchard, 2008; Packard \& Boardman, 2008). But, these views are debatable for a

775 school of defenders of the traditional protocol. For instance, Mascaro et al. (2014), stress on an

776 important drawback in findings in Packard (2013) that refuted the traditional analysis method of

777 allometry. This concerns the apparent significant bias linked to small values of the explanatory

778 variable, that result from a use of nonlinear regression with the assumption of homoscedastic

779 errors. Besides, Mascaro et al. (2014), underline that a lack of a CF misled Packard (2013),

780 thereby explaining his assertion of biased results attributed to the logarithmic transformation

781 protocol. Other practitioners have also placed a vigorous defense of this procedure, (e.g. 
782 Ballantyne, 2013; Glazier, 2013; Lai et al. 2013; White et al. 2012; Xiao et al. 2011). This is

783 reasonably understood since inferences of many allometric studies could be invalidated by a

784 substantiated rebuttal of this analysis method. But, Packard (2017a) asserts for instance, that

785 adherence to a TAMA approach has been maintained even in situations when the resulting

786 bivariate distribution is curvilinear in geometrical scale. Consequent pattern is generally referred

787 as non-log linear allometry (Packard, 2012b; Strauss \& Huxley, 1993; Echavarria-Heras et al.

788 2019). Moreover, G.C. Packard has considered deviations from linearity in log-log plots of

789 allometry as mainly attributable to a logtransformation itself (Packard \& Boardman, 2008;

790 Packard, 2012a; Packard, 2012b; Packard, 2013). From this perspective, overcoming the bias due

791 to curvature in log scale from this requires extending complexity of Huxley's model of simple

792 allometry in direct scales which bears a paradigm of multiple parameter complex allometry

793 (Gould, 1966; Lovett \& Felder, 1989; MacLeod, 2014; Bervian et al. 2006; Packard, 2012a).

794 Again, for promoters of the traditional approach this viewpoint sacrifices appreciation of

795 biological theory in order to privilege statistical correctness (Houle et al. 2011; Lemaître et al.

796 2015; Pélabon et al. 2018). The approach underwent here demonstrates that a merging of points

797 above can be achieved by evoking Huxley's report on the existence of a breakpoint in the log-

$798 \log$ plot of chela mass vs body mass of fiddler crabs (Uca pugnax) (Huxley, 1924; Huxley, 1927;

799 Huxley, 1932). A generalization of this perspective explains adoption of a polyphasic loglinear

800 allometry paradigm (Packard 2016; Gerber, Eble \& Neige 2008; Strauss \& Huxley 1993;

801 Hartnoll 1978). This notion bestows curvature in geometrical space as determined by breakpoints

802 interpreted as thresholds for transition among successive growth phases. Formally, this

803 conception adds complexity for improving statistical consistency while keeping the meanings of

804 allometric exponents as Huxley's original formulation. 
805 Conventional approaches have handled curvature in geometrical space by means of polynomial 806 regression (Kolokotrones et al. 2010; Lemaître et al. 2014; MacLeod, 2010; Glazier et al. 2013;

807 Tidière et al. 2017; Echavarria-Heras et al. 2019). Nevertheless, complexity underneath

808 precludes accounting for heterogeneity as determined by break-point allometry. Conventional

809 identification procedures also offer refined broken-line regression protocols (Beckman \& Cook, 810 1979; Ertel \& Fowlkes, 1976; Muggeo 2003; Tsuboi et al. 2018; Ramírez-Ramírez et al. 2019;

811 Echavarria-Heras et al. 2019). Nevertheless, this slant relies on nonlinear regression that requires

812 starting values for break-point estimation. Besides, crucial profile log likelihood could be log-

813 concave so local maxima problems may exist. Surpassing this inconvenience may depend on

814 using smoothed scatter plots to get candidate break points and consider several additional trials

815 for estimation sensitivity to different starting points. Also necessary inferences on estimates

816 could make implementation difficult (Julious, 2001; Muggeo, 2003).

817 The approaches in Bitar et al. (2016) and in Echavarria-Heras et al. (2018a) typify fuzzy logic

818 based hybrid paradigms aimed to allometric examination. Present TSK constructs can be placed

819 in this framework. Moreover, as our results demonstrate present fuzzy paradigm can naturally

820 host complexity as intended in a break point assimilation of allometry. Moreover, present TSK

821 arrangements offer direct-intuitive and starting value free identification of breakpoints. Certainly,

822 beak points as conceived here correspond to points of intersection of TSK-firing strength factors.

823 Besides, intervals in between break points can be interpreted as dominance realm of

824 corresponding sub models. The TSK break point identification in geometrical space for the

825 Echavarria-Heras et al. (2019) and the Mascaro et al. (2011) was paralleled by conventional

826 broken-line regression. This confirms consistent capabilities by the fuzzy paradigm to identify

827 heterogeneity in of the logtransformmed response. Thus, the offered TSK fuzzy model can be 
828 considered a tool entailing efficient automatic detection of weighted polyphasic log linear

829 allometry patterns. And, the fact that the TSK model identified linearity in geometrical space for

830 the De Robertis \& Williams (2008) data demonstrates this approach can adapt complexity as

831 necessary in an efficient way. But, we must emphasize that consistency of results in arithmetical

832 space hinges on suitability of CF form. We suggest contemplating the optimal reproducibility

833 criterion around equation (10) for this matter.

834 Motivation for the present research mainly stirred from the idea that identification based on a

835 logarithmic transformation is suited for allometric examination. Visual inspection of TSK

836 proxies fitted in geometrical space, as well as, included model performance metrics provides

837 partial validation of our assertion. But, from the perspective of MPCA proponents, validation of

838 detected heterogeneity should be made on the original arithmetic scales. Moreover, the addressed

839 TSK-MPCA proxy corresponds to an expression of the general output of the TSK fuzzy model

840 involving linear consequents in arithmetical scale. This arrangement is consistent with a MPCA

841 approach as conceived in Lovett \& Fedler (1989). Furthermore, identification of a TSK-MPCA

842 arrangement allows examination of break point allometry in arithmetical scales. Existence of

843 break points in direct scales of data, confirms that a corresponding structure detected in

844 geometrical space was not induced by effects of a logtransformation itself. And, using the

845 Weierstrass approximation theorem, it can be demonstrated that the general output of a TSK

846 fuzzy model can uniformly approximate any continuous function to arbitrarily high precision

847 (Ying 1998; Zeng et al. 2000). Therefore, the high order interpolation capabilities of the TSK-

848 MPCA scheme sets criterion to evaluate performance of retransformed TSK-PLA output $E_{\text {gTSK }}$

$849(y \mid x)$. Certainly, as our results demonstrate this can be achieved by comparing the

850 reproducibility strength of $E_{g T S K}(y \mid x)$ against that of $E_{a T S K}(w \mid a)$ for a given data set. And in the 
851 present settings the offered TSK-PLA or TSK-MPCA approaches were equally suited. This

852 demonstrates that it is possible to maintain a logtransformation as part of a consistent allometric

853 examination arrangement. This is a controversial subject whose clarification seems to be

854 overcome by adopting presently offered analytical arrangement.

855 Packard (2012a) applied conventional statistical methods to conclude that a break point in

856 Huxley's Uca pugnax data (Huxley 1932) was inexistent. Nevertheless, application of present

857 fuzzy methods detected a break point shifted back from the locus Huxley conceived.

858 Corroboration of existence of this point seems to endure a biologically meaningful interpretation

859 by Huxley of existence of a threshold for a sudden change in relative growth of the chela at about

860 the time crabs reach sexual maturity. Likewise, detected break point in Zostera marina could be

861 interpreted as a threshold beyond which plant assigns to leaves a relatively greater amount of

862 tissue to resist drag force effects that induce damage and separation from shoots. As a result,

863 allometric scaling parameters among small and large leaves could be different (Echavarria-Heras

864 et al. 2018b). Similarly, detected break point in Metrosideros polymorpha suggest that

865 differential allometric rules can be expected depending on tree size. Indeed, resource allocation

866 to different tree attributes like diameter or height can vary during growth as a response to

867 different environmental-biotic conditions, as well as, to changes in resource availability. In this

868 perspective, a risk of suppression by competitors may induce small trees to dispense more

869 resources to increase height. Then, beyond a threshold height at which suppression risk is at a

870 minimum resource may be assigned to horizontal growth parameters like diameter, crown and

871 root cover (Weiner, 2004; Ramírez-Ramírez et al. 2019). Thus, since the aim of allometric

872 examination is comprehending the biological processes that bring about covariance among traits,

873 analytical methods entailing break point identification must be preferred over conventional 
874 complex multi-parameter approaches. Indeed, on spite of any gains in statistical fit attributable to

875 the latter, characterization of inherent heterogeneity by the former could enhance biological

876 insight. Particularly, a TSK-PLA approach could be a highly biologically meaningful model of

877 allometry, because it can model the breakpoints while keeping the meanings of allometric 878 exponents as Huxley's original formulation.

879 As it is demonstrated by the steps in the derivation of equation (23), an imbedding of the TSK880 PLA in the original theoretical perspective of allometry makes MPCA in arithmetical scale its

881 logical consequence. By the same token the TSK-PLA approach grants direct-intuitive and 882 starting values-free estimation of break points for transition among growth phases. We can also 883 refer to benefits derived from the outstanding high order interpolation capabilities by this device.

884 This functional mode of the TSK paradigm can be achieved by adjusting the value of the 885 clustering parameter $r_{a}$ in equation (B7) (radii in supplied code) as to let the identification 886 algorithm increase the number $q$ of interpolation sub models in equation (20) or (48). And, if we 887 can manage to include a suitable $\mathrm{CF}$ form, we can assure a remarkable reproducibility strength of 888 projections of values of the response in arithmetical scales. Nevertheless, unsuitable forms of 889 membership functions could lead to inconveniences in the present TSK approach. Moreover, 890 fitting results of the TSK-MPCA on the Mascaro et al. (2014) data exhibit the extent on what a 891 combination of membership functions form and $r_{a}$ value can influence both break point 892 detection and reproducibility strength (Table 9). We can be aware for instance that for 893 membership functions in the form set by equation (51) consistent break point transference among 894 geometrical and arithmetical scales is only achieved when $r_{a}=0.52$ which implies

895 heterogeneity set by $q=3$. Nevertheless, this by the way leads to a penalization in 896 reproducibility strength relative to a fit by $r_{a}=0.855$. Setting a compromise between both fits 
897 depends on integration of previous knowledge into the analysis. This could help for instance by

898 suggesting ad hoc forms of membership function with the aim of achieving high reproducibility

899 and consistent break point placement relative to that previously estimated on geometrical space.

900 In any event present digression on integration of subjective knowledge in the analysis of

901 Huxley's data illustrates the extent on which a fuzzy logic approach can elucidate issues in

902 allometric examination.

903 Conclusions

904 The offered TSK-PLA as formalized by the $v_{T S K}(u, \pi)$ paradigm can be interpreted as a

905 generalized tool for the analysis of $\log$ transformed allometric data, that allows to contemplate:

906 (1) the regression arrangement of the TAMA way (the case $q=1$ and $\vartheta^{1}(u)=1$ ), (2) a

907 generalized nonlinear model for identification of weighted polyphasic nonlinear allometry (the

908 case $q>1$ ). (3) A direct-intuitive identification of concomitant break points for transition

909 among successive growth phases.

910 On spite of a seemingly complicated formal set up of the $v_{T S K}(u, \pi)$ scheme, this can be

911 conveniently identified by loading logtransformmed data into the provided code. Analysis of

912 model performance metrics show that the mean response function $\mathrm{E}_{g T S K}(y \mid \mathrm{x})$ deriving from

913 retransformation of $v_{T S K}(u, \pi)$ to arithmetical space produces similar reproducibility strength as

914 its counterpart $E_{a T S K}(y \mid x)$ following from identification of its arithmetical space TSK-MPCA

915 counterpart $w_{T S K}(x, \boldsymbol{p})$. Available conventional like broken line or weighted linear segment

916 mixture regression approaches could offer reasonable analytical paradigms. Nevertheless, the

917 offered TSK approach bears a flexible computational assembly for previous knowledge

918 integration in an intuitive-interactive way. The present digression on Huxley's break point

919 illustrates this advantage in a more proper way. 
920 Present results confirm the pertinence of the quotation of Kerkhoff \& Enquist (2009), on the

921 uselessness of a distinction between logarithmic transformations and nonlinearity in many

922 instances of allometric examination. Moreover, in our view, whenever we can manage to exhibit

923 a suitable CF form proposed Takagi Sugeno Kang generalization can elucidate a glowing

924 controversy. Surely, this paradigm allows the coexistence of the log transformation step claimed

925 by practitioners as a must in allometry, and the unbiasedness of parameter estimates attributed to

926 alternate direct nonlinear regression approaches in the original scale defended by many others.

927 But, the fact that TSK-PLA modeling provided meaningful interpretation in present

928 settings does not rear this paradigm as a general tool of allometric examination. In the

929 elucidating around equation (1) we established a condition on the response being positive and

930 having a zero limit as covariate approaches zero. Therefore, the TSK-PLA slant essentially

931 aims to analyse zero intercept allometries. And, there are instances where the initial timing of

932 development of the trait itself and overall size are different. This situation will lead to

933 consideration of a negative intercept in direct scales, ruling out transference of the examination

934 into geometrical space. Then, modeling should be necessarily kept in direct scales and relying in

935 MPCA turns out to be biologically reasonable. There are also situations where the error structure

936 can be additive while the biological process underlying allometry is multiplicative. Again, this

937 requests keeping the analyses on the arithmetic scales or modeling heteroscedastic errors in

938 geometrical space. Certainly, we briefly addressed this approach while analyzing the eelgrass

939 data. But, again offering a heteroscedastic TSK-PLA protocol suited for the general settings

940 requires further exploration.

941 Appendix A. Formal elucidation of the $w_{T S K}(x, p)$ proxy for $w(x, p)$ 
942 Azeem, Hanmandlu \& Ahmad (2000) proposed the Generalized Fuzzy Model (GFM) from

943 which the Mandami (1997) \& Larsen (1980) or the Takagi-Sugeno-Kang additive fuzzy models

944 can be derived as particular cases. And Gan, Hanmandlu \& Tan (2005) demonstrated that the

945 conditional mean of a Gaussian Mixture Model and the defuzzified output of a Generalized

946 Fuzzy Model (GFM) are mathematically equivalent. From this it follows that the probability

947 density function of a Gaussian mixture can be reduced to accommodate the TSK fuzzy model.

948 Likewise, by using the Weierstrass approximation theorem, Ying (1998) demonstrated that the

949 general output of a TSK fuzzy can uniformly approximate any continuous function to arbitrarily

950 high precision. In what follows we describe steps in the derivation of a $w_{T S K}(x, \boldsymbol{p})$ proxy for $w$

$951(x, \boldsymbol{p})$ when the former is expressed as the general output of a first order TSK fuzzy model. In

952 what follows, we review the steps in order to conceive a TSK surrogate $w_{T S K}(x, \boldsymbol{p})$ for $w(x, \boldsymbol{p})$.

953 For that aim, we recall our definition of $w(x, \boldsymbol{p})$ as a continuous function $w(x, \boldsymbol{p}): X \rightarrow Y$ with both

954 domain and range as sets of real numbers and $\boldsymbol{p}$ a set of parameters, and such that the allometric

955 response $y$ admits the representation $y=w(x, \boldsymbol{p})$.

956 In order, to elaborate on the construction of a TSK fuzzy model, we firstly need to bring up an

957 abstract structure known as a fuzzy inference system. For that aim, we consider a set a $T_{x}$

958 containing a number $q$ of linguistic terms $\Phi_{k}$ associated to the input variable $x$ (Dernoncourt,

959 2013; Mendel, 2001; Zadeh, 1989; Zadeh, 1972). Namely

$960 T_{x}=\left\{\Phi_{k} \mid k=1,2, \ldots ., q\right\}$.

961 Each linguistic term $\Phi_{k}$ is described by means a membership function $\mu_{\Phi_{k}}(x): X \rightarrow[0,1]$. Then, if

$962 x_{1}, x_{2}, \ldots, x_{n}$ are the values that $x$ takes on, the characterization

963

$A_{k}=\sum_{x \in X} \mu_{\Phi_{k}}(x) / x=\left\{\mu_{\Phi_{k}}\left(x_{1}\right) / x_{1}, . ., \mu_{\Phi_{k}}\left(x_{n}\right) / x_{n}\right\}$ 
964 is defined as a fuzzy set (Dernoncourt, 2013; Mendel, 2001; Zadeh, 1965).

965 In what follows, we use the symbol $L_{x}$ to denote the collection of membership functions

966 describing the input variable $x$, that is,

$967 L_{x}=\left\{\mu_{\Phi_{k}}(x) \mid k=1,2, \ldots, q\right\}$.

$968 L_{x}$ is known as a fuzzy partition of the input variable $x$ in the domain $X$ (Mendel, 2001;

969 Bodjanova, 1993; Bezdek, 1981).

970 In the same way, we designate a collection $T_{y}$ of linguistic terms $\Psi_{j}$ associating to the output

971 variable $y$.Namely

$972 T_{y}=\left\{\Psi_{j} \mid j=1,2, \ldots, r\right\}$.

973 Likewise, each linguistic term $\Psi_{j}$ is described by means a membership function $\mu_{\Psi_{j}}(y): Y \rightarrow$

$974[0,1]$ such that, if $y_{1}, \ldots, y_{m}$ are the values that $y$ acquires (Dernoncourt, 2013; Mendel, 2001;

975 Zadeh, 1965), then we can also consider the fuzzy set

$976 \quad B_{j}=\sum_{y \in Y} \mu_{\Psi_{j}}(y) / y=\left\{\mu_{\Psi_{j}}\left(y_{1}\right) / y_{1}, \ldots, \mu_{\Psi_{j}}\left(y_{m}\right) / y_{m}\right\}$

977 (Mendel, 2001). Concurrently, we have the collection $L_{y}$ of membership functions describing the

978 output variable $y$

979

$L_{v}=\left\{\mu_{\Psi_{j}}(y) \mid j=1,2, \ldots, r\right\}$

980 This way, the $L_{y}$ sets a fuzzy partition for the output variable $y$ in the domain $Y$ (Mendel, 2001;

981 Bodjanova, 1993; Bezdek, 1981).

982 Additionally, for $i=1,2, \ldots, q$, we advance correspondences $i \rightarrow A_{k(i)}$ and $i \rightarrow B_{j(i)}$, therefore, we

983 can contemplate antecedents $P^{i}(u)$ of the form

$984 P^{i}(x):\left[x\right.$ is $\left.A_{k(i)}\right]$ 
985 and consequents $Q^{i}(y)$

$986 Q^{i}(y):\left[y\right.$ is $\left.B_{j(i)}\right]$

987 backing inferential rules $R^{i}$

$988 R^{i}:\left\{\begin{array}{ccc}\text { if } & : & \left.P^{i}(y)\right) \\ \text { then } & : & Q^{i}(y)\end{array}\right\}$.

989 We may now think about a single input-single output fuzzy inference system (Mendel, 1979).

990 This is conceived as an application $F: X \rightarrow Y$ incorporating (1) a fuzzification module that

991 characterizes the fuzzy partitions $L_{x}$ and $L_{y},(2)$ an inference engine that uses the rules $R=$

$992 \cup_{1}^{p}\left\{R^{i}\right\}$ to convert a fuzzy input into a fuzzy output, and (3) a defuzzification operator $D$ that

993 transforms the fuzzy set obtained by the inference engine into a crisp value $y$ in $Y$.

994 A first order single input-single output Takagi-Sugeno-Kang fuzzy inference system (Sugeno \&

995 Kang, 1988; Takagui \& Sugeno, 1985) considers decision rules $R^{i}$ having an antecedent $P^{i}(x)$ of

996 the form given by equation (A7) but with the consequent $Q^{i}(y)$ in expression (A8) taking a crisp

997 functional form $f^{i}(x)$. That is, in the TSK fuzzy inference system we consider $R^{i}$ rules of the

998 form

999

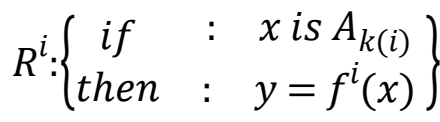

1000 for $i=1,2, \ldots, q$. We notice also that being the consequent a real number the use of a

1001 defuzzification operator is not necessary.

1002 An important component in a TSK fuzzy model is the firing strength $\varphi^{i}(x)$ of the antecedent $P^{i}$

$1003(x)$ of a rule $R^{i}$. For a first order single input-single output TSK fuzzy model we take

$1004 \varphi^{i}(x)=\mu_{\Phi_{k(i)}}(x)$.

1005 A normalized firing strength $\vartheta^{i}(x)$ takes a form (Mendel, 2001) 
$1006 \vartheta^{i}(x)=\frac{\varphi^{i}(x)}{\sum_{1}^{q} \varphi^{i}(x)}$

1007 It follows that

$1008 \sum_{1}^{q} \vartheta^{i}(x)=1$

1009 The final output $w_{T S K}(u)$ of the Takagi-Sugeno-Kang inference system is the normalized firing

1010 strength weighted average of all rule outputs (Sugeno \& Kang, 1988; Takagui \& Sugeno, 1985),

1011 that is,

$1012 w_{T S K}(x, \boldsymbol{\theta})=\sum_{1}^{q} \vartheta^{i}(x) f^{i}(x)$.

1013 Where $\boldsymbol{\theta}$ stands for the set of parameters identifying the membership and consequent functions

1014 in equations (A3) and (A10) one to one.

1015 Appendix B. Identification procedures for $\boldsymbol{w}_{T S K}(\boldsymbol{x}, \boldsymbol{p})$

1016 Description of structure and parameter estimation of the TSK fuzzy model interrelate

1017 (Echavarría-Heras et al. 2018a). A first stage relies on Subtractive Clustering (Castro et al. 2016;

1018 Chiu 1994). This technique aims to identify regions in the input space $X$ displaying high point

1019 densities. Initially a point with the highest number of neighbors is selected as the center for a

1020 group. Points in this group placed within a pre-specified fuzzy radius are removed. Then, the

1021 algorithm finds again the point with the largest number of neighbors and so on until all points in

$1022 X$ are examined. This acquires decision rules $R^{i}$. This stage also produces parameter estimates for

1023 the $\mu_{\Phi_{k}}(x)$ membership functions typifying fuzzy sets of antecedents. This determines estimated

1024 forms of the normalized firing strength factors $\vartheta^{i}(x)$. A second stage of the identification task is

1025 achieved by placing weight factors $\vartheta^{i}(x)$ in equation (A14) in order to obtain parameter 
1026 estimates for the consequents of the rules $f^{i}(x)$. Regularly, this is achieved by means of

1027 recursive least squares methods (Jang et al. 1997; Wang \& Mendel 1992).

1028 a) The Subtractive Clustering method

1029 The subtractive clustering method (SC) is an extension of the Mountain Function Clustering

1030 method (MFC) proposed originally by Yager and Filev (1994). This procedure estimates cluster 1031 centers based on the notion of a density function. For clarifying aims, before we explain the SC 1032 procedure, it is convenient to describe the MFC method. Following Yager and Filev (1994) and 1033 Chiu (1994), the MFC procedure assembles the following steps:

1034 1. The set of $n$ data to be analyzed is arranged as a vector $X$ namely:

$1035 \quad X=\left\{x_{1}, x_{2}, \ldots, x_{n}\right\}$.

1036 2. Generation a nth dimensional space grid on which the data is located. Intersections of grid 1037 lines provide nodes $N_{i} . i=1, \ldots, m$. Cluster centers to be are restricted to grid nodes. The set 1038 of cluster centers to be denotes through $C$.

1039 Based on the distribution of the data devise a mountain function (MF). This represents a data 1040 density measure. The height of the mountain function at a node point $N_{i}$ is

1041 $M F\left(N_{i}\right)=\sum_{k=1}^{n} e^{-\alpha d\left(x_{k}, N_{i}\right)}, i=1,2$, where $\alpha$ is a positive constant and $d\left(x_{k}, N_{i}\right)$ is a measure of the distance between data point $1043 x_{k}$ and grid node $N_{i}$. Equation (B2) enunciates that the data density measure at a point $N_{i}$ is 1044 influenced by all data points $x_{k}$. Such a density measure varies inversely proportional to 1045 distance between data points and node $N_{i}$. The parameter $\alpha$ not only influences the maximum $1046 \quad$ value of $M F\left(N_{i}\right)$ but also its smoothness. 
1047 It can be ascertained that the values of the mountain function on nodes are closely dependent 1048 on density of data points in the neighborhood of $N_{i}$. Mountain function values can be also 1049 interpreted as the potential suitability of a grid node to become a cluster center estimate. A 1050 node with many neighboring data points will have a large mountain function value.

1051 Cluster center $C_{1}$ is chosen as node $N_{i}$ such that $M F\left(N_{i}\right)$ attains the maximum value among 1052 all remaining nodes, formally

1053 $M F^{1}\left(C_{1}\right)=\max _{i}\left[M F\left(N_{i}\right)\right]$

1054 Since the nodes close to $C_{1}$ will also have high mountain-function values, it deems necessary to remove the effect of $C_{1}$ before obtaining the next cluster center $C_{2}$. A new mountain function $M F^{2}\left(N_{i}\right)$ is shaped by taking off a scaled Gaussian function centered at $C_{1}$ this eliminates the effect of the first cluster. Iteratively the mountain function, after eliminating the effects of the cluster center that was previously identified becomes

3. The previous step is repeated until the number of desired cluster centers is found or until a stopping condition is met. This expresses in terms of the ratio of first maximum value of

$$
M F^{k+1}\left(C_{k+1}\right)=\max \left[M F^{k}\left(N_{i}\right)-M F^{k}\left(C_{k}\right) \sum_{k=1}^{n} e^{-\beta d\left(C_{k}-N_{i}\right)}, 0\right]
$$
mountain function found $M F^{1}\left(C_{1}\right)$ to corresponding penultimate maximum value found $M F^{k-1}\left(C_{k-1}\right)$. Iteration stops when this ratio attains a value less than a certain positive constant $\delta$,

$$
\frac{M F^{1}\left(C_{1}\right)}{M F^{k-1}\left(C_{k-1}\right)}<\delta
$$

1066 Since, the mountain function has to be evaluated at each grid point the processing time of the 1067 mountain clustering method rises exponentially with dimension of task. A SC scheme amends 
1068 these difficulties by taking data points as potential cluster centers. This implies processing time

1069 becoming proportional to problem size instead of problem dimension. Since this method does not

1070 take into account any grid intersections execution time is reduced. Nevertheless, the real clusters

1071 centers do not necessarily place at data points, but in most cases this approach offers a good

1072 approximation, to cluster center identification. The SC approach also bases on a function

1073 representing the distribution of the data. Actually, the SC method consists of equations very

1074 similar to those used in the MF method, steps in the later are:

1075 1. The set of $n$ data to be analyzed is defined as follows:

$1076 \quad X=\left\{x_{1}, x_{2}, \ldots, x_{n}\right\}$

1077 2. Since, each data point is an aspirant for a cluster center a density measure at data point $x_{i}$ is

1078 defined as

$1079 \quad D_{i}=\sum_{j=1}^{n} \exp \left(-\frac{d\left(x_{i}, x_{j}\right)}{\left(r_{a} / 2\right)^{2}}\right)$

1080 where $r_{a}$ is a positive constant, this constant acts as the radius that defines the area of

1081 proximity to the potential cluster center. Once all density assessments are obtained, the one

1082 with the highest value is taken. Formally

$1083 \quad C_{1}=\operatorname{Max}\left[D_{i}\right]$

1084 this will identify a first cluster center $C_{1}$,

1085 3. Next cluster center is acquired by subtracting a scaled Gaussian function centered at $C_{1}$, that 1086 is

$1087 \quad D_{i}=D_{i}-C_{1} \exp \left(-\frac{d\left(x_{i}, C_{1}\right)}{\left(r_{b} / 2\right)^{2}}\right)$

where $r_{b}<r_{a}$, is a radius that defines the proximity area of the cluster center $C_{1}$. 
1089 4. The maximum value of rescaled densities $D_{i}$ of equation (B9) is chosen as cluster center $C_{2}$.

1090 This procedure is repeated until a desired number $m$ of cluster centers $C_{1}, C_{2}, \ldots . C_{m}$ is

1091 determined.

1092 b) The Recursive Least Squares method

1093 The general least-squares problem establishes the output of a model $y$ as given by a linearly

1094 parameterized expression, namely

$1095 y=\gamma_{1} h_{1}(\boldsymbol{x})+\gamma_{2} h_{2}(\boldsymbol{x})+\cdots+\gamma_{n} h_{n}(\boldsymbol{x})$,

1096 where $\boldsymbol{x}=\left[x_{1}, \cdots, x_{p}\right]^{T}$ is the model's input values vector, $h_{1}(\boldsymbol{x}), \cdots, h_{n}(\boldsymbol{x})$ are known functions of

$1097 \boldsymbol{x}$, and $\gamma_{1}, \cdots, \gamma_{n}$ called regression coefficients are to be fitted.

1098 Without loss of generality, we address the case $q=2$ assuming consequent linear functions in

1099 the form given by equation (53), so that the general output of the TKS of equation (A14) is

$1100 w_{T S K}(x, \boldsymbol{\theta})=p_{1}^{1} \vartheta^{1}(x) x+p_{1}^{2} \vartheta^{2}(x) x+p_{2}^{1} \vartheta^{1}(x)+p_{2}^{2} \vartheta^{2}(x)$.

1101 with $\vartheta^{i}(x)$ given by equation (A12). Then, $w_{T S K}(x, \boldsymbol{\theta})$ as given by equation (B11) becomes a

1102 particular characterization of equation (B10) by taking model's input values $x=[x]^{T}$, and $p_{1}^{1}$,

$1103 p_{2}^{1}, p_{1}^{2}$ and $p_{2}^{2}$ unknown parameters in the consequent functions.

1104 To obtain parameter estimates, we take into account that in the present settings the target system

1105 to be modeled involves an input-output relationship $x \rightarrow w_{T S K}(x, \boldsymbol{\theta})$ being $x$ the descriptor

1106 variable and $w_{T S K}(x, \boldsymbol{\theta})$ standing for the response $y$. Therefore, we have a training data

1107 composing pairs $\left(x_{k}: y_{k}\right)$, for $k=1, \cdots, m$ that stand for replicates of the considered input-output

1108 relationship. Therefore, in order to identify the unknown parameters $p_{1}^{1}, p_{2}^{1}, p_{1}^{2}$ and $p_{2}^{2}$, we must

1109 fill in for each data pair $\left(x_{k}: y_{k}\right)$, into equation (B11) in order to obtain the set of $m$ linear

1110 equations: 
1111

$$
\left\{\begin{array}{ccc}
p_{1}^{1} \vartheta^{1}\left(x_{1}\right) x_{1}+p_{1}^{2} \vartheta^{2}\left(x_{1}\right) x_{1}+p_{2}^{1} \vartheta^{1}\left(x_{1}\right)+p_{2}^{2} \vartheta^{2}\left(x_{1}\right) & = & y_{1} \\
\vartheta^{1}\left(x_{2}\right) p_{1}^{1} x_{2}+p_{1}^{2} \vartheta^{2}\left(x_{2}\right) x_{2}+p_{2}^{1} \vartheta^{1}\left(x_{2}\right)+p_{2}^{2} \vartheta^{2}\left(x_{2}\right) & = & y_{2} \\
\vdots & \vdots & \vdots \\
\vartheta^{1}\left(x_{m}\right) p_{1}^{1} x_{m}+p_{1}^{2} \vartheta^{2}\left(x_{m}\right) x_{m}+p_{2}^{1} \vartheta^{1}\left(x_{m}\right)+p_{2}^{2} \vartheta^{2}\left(x_{m}\right) & = & y_{m}
\end{array}\right\}
$$

1112 This system of equations can be equivalently written in a concise form $\boldsymbol{B} \boldsymbol{P}=\boldsymbol{y}$, where $\boldsymbol{B}$ is the $1113 m \times n$ matrix,

$1114 \quad \boldsymbol{B}=\left\{\begin{array}{cccc}\vartheta^{1}\left(x_{1}\right) x_{1} & \vartheta^{1}\left(x_{1}\right) & \vartheta^{2}\left(x_{1}\right) x_{1} & \vartheta^{2}\left(x_{1}\right) \\ \vartheta^{1}\left(x_{2}\right) x_{2} & \vartheta^{1}\left(x_{2}\right) & \vartheta^{2}\left(x_{2}\right) x_{2} & \vartheta^{2}\left(x_{2}\right) \\ \vartheta^{1}\left(x_{m}\right) x_{m} & \vartheta^{1}\left(x_{m}\right) & \vartheta^{2}\left(x_{m}\right) x_{m} & \vartheta^{2}\left(x_{m}\right)\end{array}\right\}$

$1115 \boldsymbol{P}$ the $n \times 1$ vector of unknown parameters,

$1116 \quad \boldsymbol{P}=\left\{\begin{array}{l}p_{1}^{1} \\ p_{2}^{1} \\ p_{1}^{2} \\ p_{2}^{2}\end{array}\right)$

1117 and being $\boldsymbol{y}$ the $m \times 1$ output values vector:

$1118 \boldsymbol{y}=\left\{\begin{array}{c}y_{1} \\ y_{2} \\ \vdots \\ y_{m}\end{array}\right\}$

1119 The i-th row of the data matrix $[\boldsymbol{B} \vdots \boldsymbol{y}]$ is denoted by $\left[\boldsymbol{b}_{\boldsymbol{i}}^{\boldsymbol{T}}, \boldsymbol{y}_{\boldsymbol{i}}\right]$ and formally represented by,

$1120 \quad \boldsymbol{b}_{\boldsymbol{i}}^{\boldsymbol{T}}=\left[\begin{array}{llll}\vartheta^{1}\left(x_{i}\right) x_{i} & \vartheta^{1}\left(x_{i}\right) & \vartheta^{2}\left(x_{i}\right) x_{i} & \vartheta^{2}\left(x_{i}\right)\end{array}\right]$

1121 Then, equation (B12) modifies to include an error vector $\boldsymbol{e}$ that accounts for random noise or

1122 modeling error, that is,

$1123 \boldsymbol{y}=\boldsymbol{B P}+\boldsymbol{e}$.

1124 Since $\boldsymbol{e}=\boldsymbol{y}-\boldsymbol{B P}$ then $\boldsymbol{e}^{\boldsymbol{T}} \boldsymbol{e}=(\boldsymbol{y}-\boldsymbol{B P})^{\boldsymbol{T}}(\boldsymbol{y}-\boldsymbol{B P})$, and if we let $\boldsymbol{E}(\boldsymbol{P})=\boldsymbol{e}^{\boldsymbol{T}} \boldsymbol{e}$ we will have 
$1125 \boldsymbol{E}(\boldsymbol{P})=\sum_{i=1}^{m}\left(\boldsymbol{y}_{\boldsymbol{i}}-\boldsymbol{b}_{\boldsymbol{i}}^{\boldsymbol{T}} \boldsymbol{P}\right)^{2}$.

1126 We call $\boldsymbol{E}(\boldsymbol{P})$ the sum of squared errors. Then, we need to search for a characterization $\hat{\boldsymbol{P}}$ of the

1127 vector $\boldsymbol{P}$, which minimizes $\boldsymbol{E}(\boldsymbol{P})$. Furthermore, the vector $\hat{\boldsymbol{P}}$ is known as the least-squares

1128 estimator (LSE) of $\boldsymbol{P}$. Since $\boldsymbol{E}(\boldsymbol{P})$ is in quadratic form, $\hat{\boldsymbol{P}}$ is unique. It turns out that $\hat{\boldsymbol{P}}$ satisfies

1129 the normal equation

$1130 \boldsymbol{B}^{\boldsymbol{T}} \boldsymbol{B} \hat{\boldsymbol{P}}=\boldsymbol{B}^{\boldsymbol{T}} \boldsymbol{y}$.

1131 Furthermore, $\hat{\boldsymbol{P}}$ is given by

$1132 \hat{\boldsymbol{P}}=\left(B^{T} B\right)^{-1} B^{T} y$

1133 A $n$-order least squares estimator $\hat{\boldsymbol{P}}_{\boldsymbol{n}}$ of $\hat{\boldsymbol{P}}$ defined by means of the expression

$\hat{P}_{n}=\left(B^{T} B\right)^{-1} B^{T} y$

1135 is a description of $\hat{\boldsymbol{P}}$ that associates to $n$ data pairs taken out of the training data set $\left(x_{i}: y_{i}\right)$. Once

1136 we have gotten $\hat{\boldsymbol{P}}_{\boldsymbol{n}}$ we can acquire the following estimator $\hat{\boldsymbol{P}}_{\boldsymbol{n}+\mathbf{1}}$ with a minimum of effort,

1137 through a recursive least-squares estimator (RLSE) technique, a procedure where the $n t h$ row of

$1138[\boldsymbol{B}: \boldsymbol{y}]$, with $(1 \leq n \leq m)$ denoted by $\left[\boldsymbol{b}_{\boldsymbol{n}}^{\boldsymbol{T}}: \boldsymbol{y}_{\boldsymbol{n}}\right]$ is recursively obtained. We now explain the

1139 procedure behind the RLSE method.

1140 A new pair $\left(b_{n+1}^{T} ; y_{n+1}\right)$ becomes available as the $(n+1)^{\text {th }}$ entry in the data set, producing the

$1141 \hat{\boldsymbol{P}}_{\boldsymbol{n}+\mathbf{1}}$ estimate,

1142

$\hat{\boldsymbol{P}}_{n+1}=\left(\left[\begin{array}{c}B \\ b_{\boldsymbol{n}+1}\end{array}\right] T\left[\begin{array}{c}\boldsymbol{B} \\ \boldsymbol{b}_{\boldsymbol{n}+\mathbf{1}}\end{array}\right]\right)-1\left[\begin{array}{c}\boldsymbol{B} \\ \boldsymbol{b}_{\boldsymbol{n}+\mathbf{1}}\end{array}\right] T\left[\begin{array}{c}\boldsymbol{y} \\ \boldsymbol{y}_{\boldsymbol{n}+\mathbf{1}}\end{array}\right]$.

1143 Further, in order to simplify the notation, the pair $\left(b_{n+1}^{T} ; y_{n+1}\right)$ will be symbolized by $\left(b^{T} ; y\right)$

1144 and we also introduce the $p \times p$ matrices $\boldsymbol{H}_{\boldsymbol{n}}$ and $\boldsymbol{H}_{\boldsymbol{n}+\mathbf{1}}$ defined by means of

$1145 \boldsymbol{H}_{n}=\left(\boldsymbol{B}^{T} \boldsymbol{B}\right)^{-1}$, 
1146 and

$1147 \quad H_{n+1}=\left(\left[\begin{array}{c}B \\ b^{T}\end{array}\right]^{T}\left[\begin{array}{c}B \\ b^{T}\end{array}\right]\right)-1$

1148 or equivalently

$1149 \quad H_{n+1}=\left(B^{T} B+b b^{T}\right)^{-1}$.

1150 Then $\boldsymbol{H}_{\boldsymbol{n}}$ and $\boldsymbol{H}_{\boldsymbol{n}+\mathbf{1}}$ are related through

$1151 \quad H_{n+1}=\left(H_{n}^{-1}+b b^{T}\right)^{-1}$

1152 Therefore, using $\boldsymbol{H}_{\boldsymbol{n}}$ from equation (B23) and $\boldsymbol{H}_{\boldsymbol{n}+\mathbf{1}}$ from equation (B25), we explain why

1153 equations (B21) and (B22) can be equivalently written in the form

$1154 \hat{\boldsymbol{P}}_{n}=\boldsymbol{H}_{n} \boldsymbol{B}^{T} \boldsymbol{y}$

1155 and

$1156 \hat{\boldsymbol{P}}_{n+1}=H_{n+1}\left(B^{T} y+b y\right)$.

1157 From equation (B26) we have $\boldsymbol{B}^{\boldsymbol{T}} \boldsymbol{y}=\boldsymbol{H}_{\boldsymbol{n}}^{-\mathbf{1}} \hat{\boldsymbol{P}}_{\boldsymbol{n}}$, then replacing this result in equation (B27) we get

$1158 \hat{\boldsymbol{P}}_{n+1}=\boldsymbol{H}_{n+1}\left(\boldsymbol{H}_{n}^{-1} \hat{\boldsymbol{P}}_{n}+\boldsymbol{b y}\right)$.

1159 Now, from equation (B25) we have $\boldsymbol{H}_{n}^{-1} \hat{\boldsymbol{P}}_{\boldsymbol{n}}=\left(\boldsymbol{H}_{\boldsymbol{n}+\mathbf{1}}^{-\mathbf{1}}-\boldsymbol{b} \boldsymbol{b}^{\boldsymbol{T}}\right) \hat{\boldsymbol{P}}_{\boldsymbol{n}}$, so replacing this result in the

1160 above expression we get

$1161 \hat{P}_{n+1}=H_{n+1}\left[\left(H_{n+1}^{-1}-b b^{T}\right) \hat{P}_{n}+b y\right]$,

1162 then simplifying yields

$1163 \hat{\boldsymbol{P}}_{n+1}=\hat{\boldsymbol{P}}_{n}+\boldsymbol{H}_{n+1} b\left(\boldsymbol{y}-\boldsymbol{y}^{T} \hat{\boldsymbol{P}}_{n}\right)$.

1164 Thus $\hat{\boldsymbol{P}}_{\boldsymbol{n}+\mathbf{1}}$ can be recursively identified in terms of the preceding estimate $\hat{\boldsymbol{P}}_{\boldsymbol{n}}$ and the new data

1165 pairs $\left(b^{T} ; y\right)$. Furthermore, the current estimate $\hat{\boldsymbol{P}}_{\boldsymbol{n}+\mathbf{1}}$ is expressed as the previous one $\hat{\boldsymbol{P}}_{\boldsymbol{n}}$ plus a

1166 correcting term based on the new data $\left(\boldsymbol{b}^{\boldsymbol{T}} ; \boldsymbol{y}\right)$; this adjusting term can be understood as an 
1167 adaptation gain vector $\boldsymbol{H}_{\boldsymbol{n}+\mathbf{1}}$ multiplied by a prediction error $\left(\boldsymbol{y}-\boldsymbol{b}^{\boldsymbol{T}} \hat{\boldsymbol{P}}_{\boldsymbol{n}}\right)$ linked to the previous

1168 estimator $\hat{\boldsymbol{P}}_{\boldsymbol{n}}$

1169 Calculating $\boldsymbol{H}_{\boldsymbol{n}+\mathbf{1}}$ as given by equation (B24) is computationally costly and requires the

1170 adaptation of a recursive formula. From equation (B25) we have

$1171 H_{n+1}=\left(H_{n}^{-1}+b b^{T}\right)^{-1}$

1172 Using the matrix inversion formulation of Lemma 5.6 in Jang et al. (1997) with $\boldsymbol{A}_{\boldsymbol{n}}=\boldsymbol{H}_{\boldsymbol{n}}^{-\mathbf{1}}, \boldsymbol{B}=\boldsymbol{b}$

1173 , and $\boldsymbol{C}=\boldsymbol{b}^{\boldsymbol{T}}$, we obtain the successive recursive formula for $\boldsymbol{H}_{\boldsymbol{n}+\mathbf{1}}$ in terms of $\boldsymbol{H}_{\boldsymbol{n}}$ :

1174

$H_{n+1}=H_{n}-H_{n} b\left(I+b^{T} H_{n} b\right)^{-1} b^{T} H_{n}$

1175 equivalently,

$1176 \quad H_{n+1}=H_{n}-\frac{H_{n} b b^{T} H_{n}}{I+b^{T} H_{n} b}$

1177 Summarizing, the recursive least-squares estimator for the problem of $\boldsymbol{A P}+\boldsymbol{e}=\boldsymbol{y}$., where the

$1178 n t h(1 \leq n \leq m)$ row of $[\boldsymbol{B} \vdots \boldsymbol{y}]$, denoted by $\left[b_{n}^{T}: y_{n}\right]$, is sequentially obtained. It can be calculated

1179 as follows:

$1180\left\{\begin{array}{c}\hat{\boldsymbol{P}}_{n+1}=\hat{\boldsymbol{P}}_{n}+H_{n+1} \boldsymbol{b}_{n+1}\left(w_{n+1}-b_{n+1}{ }_{n}^{T} \hat{\boldsymbol{P}}_{n}\right) \\ H_{n+1}=H_{n}-\frac{H_{n} b_{n+1} b_{n+1}^{T} H_{n}}{I+b_{n+1}{ }^{T} H_{n} b_{n+1}}\end{array}\right.$

1181 Notice that in stablishing this result we have recalled equation (B30) and the fact that we had

1182 previously set the convention that for easy of presentation, the pair $\left(b_{n+1}^{T} ; y_{n+1}\right)$ would be

1183 symbolized by the expression $\left(b^{T} ; y\right)$.

1184 c) genfis $2 . m+$ anfis.m training

1185 Examination in geometrical space is achieved by the code: main_fun_tsk_pla_model_fit.

1186 Similarly, analysis in direct arithmetical scales relies on the: main_fun_tsk_mpca_model_fit 
1187 counterpart, both based on a genfis $2 . m+$ anfis.m training (both functions are included into the

1188 code in the supplementary files section). As it is explained in Matlab user's manual for genfi2.m 1189 and anfis.m functions, given sets of input and output data, genfis2 produces a Fuzzy Inference

1190 System (FIS). Output by genfis2 suggest a primary FIS for anfis.m training. This is achieved 1191 through subtractive clustering. This is achieved by genfis $2 . m$ by means the subclust.m function 1192 that extracts a set of rules that model the behavior of the data. Once the antecedent membership 1193 functions are obtained the procedure uses RLS estimation to determine the consequent functions 1194 of each rule.

1195 For the given input-output data the genfis2.m function produces a FIS of a TSK type. Moreover, 1196 the XIN and XOUT matrices yield one column per input and output of the FIS, respectively.

1197 radii (e.g. $r_{a}$ in Eq. (B7)) specifies the influence range or proximity area of the cluster center for 1198 each input and output dimension, assuming that the data falls within a hypercube unit (range [0 1199 1]). Specifying a smaller cluster radius will generally produce smaller clusters in the data and, 1200 therefore, more rules. When radii is a scalar, it applies to all input and output dimensions. When 1201 radii is a vector it has an input for each input and output dimension (Chiu, 1994).

1202 The descending gradient method is one of the oldest techniques to minimize, a given function 1203 defined in a multidimensional input space. This method bases many direct optimization methods 1204 for restricted and unrestricted problems. On spite of its slow convergence, its simplicity makes it 1205 the most used non-linear optimization technique. Formally

$1206 \boldsymbol{\varphi}_{\text {next }}=\boldsymbol{\varphi}_{\text {now }}-\eta \boldsymbol{G}$

1207 A slightly different formulation of equation (1) results from a gradient normalization, that is

$1208 \boldsymbol{\varphi}_{\text {next }}=\boldsymbol{\varphi}_{\text {now }}-\kappa \frac{\boldsymbol{G}}{\|\boldsymbol{G}\|}$ 
1209 being $\kappa$ the actual size of step, which interprets as the Euclidean transition distance from $\boldsymbol{\varphi}_{\text {now }}$ to

$1210 \boldsymbol{\varphi}_{\text {next }}$, namely

$1211 \kappa=\left\|\boldsymbol{\varphi}_{\text {next }}-\boldsymbol{\varphi}_{\text {now }}\right\|$

1212 In order to typify equations (B32) and (B33) the former one refers as simple descendent gradient 1213 and the later as its normalized version.

1214 The term $\eta \boldsymbol{G}$ in (1) stands for the extent of step. With a fixed $\eta$, step magnitude changes

1215 automatically in each iteration due to different gradients of $\boldsymbol{G}$. If the minimum point is on a flat

1216 surface or plateau, $\boldsymbol{G}$ tends to be infinitesimally small. Consequently, the simple descendent

1217 gradient in equation (1) exhibits a slow convergence. On the other hand, for a fixed $\kappa$ the

1218 normalized simple descending gradient in equation (2) always does the same steps, neglecting

1219 how steep the slope is (Chan \& Fallside, 1987; Rumlhart et al. 1986). It is then necessary to

1220 actualize step size $\kappa$ for efficiency.

1221 c) Actualizing the $\kappa$ value

1222 Adjusting $\mathbf{\kappa}$ dynamically requires an adaptive strategy. Based on empirical observations, an

1223 initial step size $\kappa=0.01$ can be updated according to the following couple of heuristic rules

1224 (Jang, 1993):

1225 1. If the objective function (MRSE) undergoes m consecutive reductions, increase by $\mathrm{p} \%$.

$1226 \operatorname{IF~} \operatorname{SSE}\left(\boldsymbol{\varphi}_{\text {next }}\right)<\operatorname{SSE}\left(\boldsymbol{\varphi}_{\text {now }}\right) \operatorname{THEN} \kappa=\kappa * \kappa_{\text {inc }}$

1227 2. If the objective function (MRSE) manifests $n$ consecutive combinations of an increase and a 1228 decrease, decrease by $q \%$.

1229

$\operatorname{IF} \operatorname{MRSE}\left(\boldsymbol{\varphi}_{\text {next }}\right) / \operatorname{MRSE}\left(\boldsymbol{\varphi}_{\text {now }}\right)>1.04(\operatorname{maxSSEinc}) \operatorname{THEN} \kappa=\kappa * \kappa_{\text {dec }}$

1230 Representative values for $\mathrm{m}, \mathrm{n}, \mathrm{p}$ and $\mathrm{q}$ are $4,2,10 \%\left(\kappa_{i n c}=1.1\right)$ and $10 \%\left(\kappa_{\text {dec }}=0.9\right)$,

1231 respectively. These typical values are more or less arbitrarily chosen. This update strategy is 
1232

1233

1234

1235

1236

1237

1238

1239

1240

1241

1242

1243

1244

1245

1246

1247

1248

1249

1250

1251

1252

1253

$\boldsymbol{\rho}_{\boldsymbol{C}}=\frac{2 \boldsymbol{\rho} \sigma_{Y} \sigma_{X}}{\left(\mu_{X}-\mu_{Y}\right)^{2}+\sigma_{Y}^{2}+\sigma_{Y}^{2}}$

1254 entries through

$A I C=-2 l(\hat{\theta})+2 p$

incorporated into hybrid learning (anfis.m: descending gradient to update the parameters in the antecedents and RLS to update the parameters of the linear consequents).

Summarizing, the radii specifies the range of influence of the cluster centers (membership function centers (e.g. $\boldsymbol{\theta}$ vector in Eq. (21)) for each input and output dimension. Specifying a smaller cluster radius will generally produce smaller clusters in the data and, therefore, more rules. The value of $\lambda$ in a membership function is related to the radii value and the range of

$\lambda=(\operatorname{radii} \cdot *$ Range $(\mathrm{X})) / \operatorname{sqrt}(8.0)$

(Chiu, 1994). The $\kappa$ in the descendent gradient method adapts the $\boldsymbol{\theta}$ and $\boldsymbol{\lambda}$ values of the membership functions (cf. Eq. (21) and Eq. (52)) in the antecedents of each iteration or epoch while minimizing the objective function.

\section{Appendix C. Model performance metrics}

In addition to the AIC and $\rho$ indices, model assessment here is mainly based on the SEE, MPE and MPSE indices that rely in on statistics of squared and absolute deviations of observed to predicted values. According to Parresol (1999), the use of SEE, MPE and MPSE statistics as model performance metrics were first recommended by Schlaegel (1982) and have subsequently been used by Zeng (2011b). We provide ahead related formulae and explanation.

\section{Akaike information criterion (AIC)}

\section{Lin's Concordance Correlation Coefficient $\left(\rho_{C}\right)$}

with $\rho$ standing for Pearson's correlation coefficient. The $\rho_{C}$ index estimates through 
$1255 \hat{\rho}_{C}=\frac{2 S_{Y X}}{(\bar{Y}-\bar{X})^{2}+S_{Y}^{2}+S_{X}^{2}}$

1256 where

$1257 \bar{Y}=\frac{1}{n} \sum y_{i}, \bar{X}=\frac{1}{n} \sum x_{i}$,

$1258 S_{Y}^{2}=\frac{1}{n} \sum\left(y_{i}-\bar{y}\right)^{2}, S_{X}^{2}=\frac{1}{n} \sum\left(x_{i}-\bar{x}\right)^{2}$,

$1259 S_{X Y}=\frac{1}{n} \sum\left(x_{i}-\bar{x}\right)\left(y_{i}-\bar{y}\right)$.

1260 Determination coefficient $\left(\boldsymbol{R}^{2}\right)$

$1261 \quad R^{2}=\frac{\sum\left(\hat{Y}_{i}-\bar{Y}\right)^{2}}{\sum\left(Y_{i}-\bar{Y}\right)^{2}}$

1262 Standard error of estimation (SEE)

$1263 S E E=\sqrt{\sum\left(y_{i}-\hat{y}_{i}\right)^{2} /(n-p)}$

1264 Mean prediction error (MPE)

$1265 M P E=t_{\alpha}(S E E / \bar{y}) / \sqrt{n} \times 100$

1266 Mean percent standard error (MPSE)

$1267 M P S E=\frac{1}{n} \sum\left|\left(y_{i}-\hat{y}_{i}\right) / \hat{y}_{i}\right| \times 100$

1268 The AIC index allows comparing performance of different candidate models that fit a set of data.

1269 The model with the lowest AIC value is considered the best among competitors. The AIC index

1270 establishes a compromise between the goodness of fit of a model and its complexity, which

1271 express through linked log-likelihood and number of parameters as a way to penalize inclusion

1272 of unnecessary ones. As it bases on information entropy an AIC index is often interpreted, as an

1273 estimate of the information lost when a model is used to represent the process that generates the

1274 data. Lin's Concordance Correlation Coefficient $\left(\rho_{C}\right)$ also symbolized through (CCC) measures

1275 how well one variable $(\mathrm{Y})$ reproduces another $(\mathrm{X})$, that is, it represents a measure of the 
1276 similarity (or agreement) between the two variables. This index can be estimated, with sample

1277 sizes of at least ten pairs $(\mathrm{x}, \mathrm{y})$. The $\mathrm{R}$ square index $\left(R^{2}\right)$ also called determination coefficient

1278 interprets through the ratio (SS due to regression/Total SS corrected for the mean) and is mainly

1279 intended as a measure of closeness between response values and adjusted linear regression

1280 models. This index also measures the proportion of the total variation of the response, around the

1281 average, explained by the model. The coefficient of determination takes values between zero and

1282 one. When $R^{2}$ attains its maximum value, one, the response variable is fully explained by the

1283 predictive variables of the fitted linear regression model. According to Parresol (1999) using the

1284 coefficient of determination a fit index aimed to compare performance of biomass models was

1285 firstly suggested by Schlaegel (1982). Nevertheless, for nonlinear models a high $R^{2}$ value does

1286 not necessarily associates with high reproducibility strength. The standard error of estimation

1287 SEE is of widespread use in statistical texts and also widely reported in statistical software. This

1288 index bears a global assessment of goodness of fit of a model to observed data, as it measures the

1289 accuracy of $\left(\hat{y}_{i}\right)$ predictions produced by a fitted regression model. This index takes non-negative

1290 values. When SEE attains its minimum value, of zero, the observed values of the response

1291 coincide with the fitted mean response function, meaning that the model displays exact

1292 reproducibility of observed values.

1293 The MPE, which is now used to determine the goodness of fit of a model, is a standardized

1294 version of the coefficient of variation $C V=(S E E / \bar{y}) \times 100$ expressed as a percentage, as

1295 proposed by Schlaegel (1982). The MPSE bears a measure of the average absolute relative error,

1296 expressed as a percentage. This model assessment index recommended by Sclaegel (1982), was

1297 previously suggested by Meyer (1938), as a measure of the absolute deviation of the expected 
1298 and predicted responses, relative to the size of the prediction $\left(\left|y_{i}-\hat{y}_{i}\right| \hat{y}_{i}\right)$ expressed as a

1299 percentage average.

1300 Acknowledgements

1301 Professor G.C. Packard and two anonymous reviewers provided valuable comments and

1302 criticism that improved our final presentation. We thank A. De Robertis for data sharing.

1303 References

1304 Ajili F, Wallace MG. 2004. Hybrid Problem Solving in ECLiPSe. In: Milano M. (eds) 1305 Constraint and Integer Programming. Operations Research/Computer Science 1306 Interfaces Series, vol 27. Springer, Boston, MA.

1307 Akaike H. 1974. A new look at the statistical model identification. IEEE Transactions on $1308 \quad$ Automatic Control 19(6):716-723.

1309 Al-Kaysi AM, Al-Ani A, Loo CK, Powell TY, Martin DM, Breakspear M, Boonstra TW. 2017.

1310 Predicting tDCS treatment outcomes of patients with major depressive disorder using 1311 automated EEG classification. Journal of Affective Disorders 208: 597-603.

1312 Alur R, Courcoubetis C, Halbwachs N, Henzinger TA, Ho P, Nicollin X, Olivero A, Sifakis J, 1313 Yovine S. 1995. The algorithmic analysis of hybrid systems. Theoretical Computer $1314 \quad$ Science, 138:3-34.

1315 Anderson TW, Darling DA. 1952. Asymptotic theory of certain "goodness-of-fit" criteria based 1316 on stochastic processes. Annals of Mathematical Statistics 23:193-212.

1317 Azeem MF, Hanmandlu M, Ahmad N. 2000. Generalization of adaptive neuro-fuzzy inference 1318 systems. IEEE Transactions Neural Networks 11(6):1332-1346.

1319 Baldwin JF, Martin T, Azvine, B. 1998. Soft Computing for Intelligent Knowledge-based Systems. BT Technology Journal 16(3): 165.179 
1321 Ballantyne F. 2013. Evaluating model fit to determine if logarithmic transformations are necessary

1322

1323

1324

1325

1326

1327

1328

1329

1330

1331

1332

1333

1334

1335

1336

1337

1338

1339

1340

1341

1342

1343

in allometry: a comment on the exchange between. Journal of Theoretical Biology 317:418-21.

Baskerville G. 1972. Use of logarithmic regression in the estimation of plant biomass. Canadian Journal of Forest Research 2: 49-53.

Beckman RJ, Cook RD. 1979. Testing for two-phase regressions. Technometrics 21:65-69.

Bello R, Verdegay JL. 2012. Rough sets in the Soft Computing environment. Information Sciences 212: 1-14

Bervian G, Fontoura N, Haimovici M. 2006. Statistical model of variable allometric growth: otolith growth in Micropogonias furnieri (Actinopterygii, Sciaenidae). Journal of Fish Biology 68:196-208.

Bezdek J. 1981. Pattern Recognition with Fuzzy Objective Function Algorithms. New York: Plenum Press.

Bezdek JC, Pal SK. 1992. Fuzzy Models for Pattern Recognition. New York: IEEE Press.

Bitar SD, Campos CP, Freitas CEC. 2016. Applying fuzzy logic to estimate the parameters of the length-weight relationship. Brazilian Journal of Biology 76(3): 611-618.

Bodjanova S. Fuzzy Sets and Fuzzy Partitions. 1993. In: Opitz O, Lausen B, Klar R, editors. Information and Classification. Studies in Classification, Data Analysis and Knowledge Organization. Heidelberg: Springer-Verlag Berlin.

Bonissone PP, Yu-To C, Goebel K, Khedkar PS. 1999. Hybrid soft computing systems: industrial and commercial applications. Proceedings of the IEEE 87(9):1641-1667.

Breusch TS, Pagan AR. 1979. A Simple Test for Heteroscedasticity and Random Coefficient Variation. Econometrica 47:1287-1294. 
1344 Castro JR. Castillo O, Sanchez MA, Mendoza O, Rodríguez-Díaz A, Melin P. 2016. Method for $1345 \quad$ Higher Order polynomial Sugeno Fuzzy Inference Systems. Information Science 351:761346 89.

1347 Chan, L.W. and Fallside F. An adaptive training algorithm for back propagation networks. $1348 \quad$ Computer Speech and Languaje, 2:205-218, 1987

1349 Chen Y. 2001. Hybrid soft computing approach to identification and control of nonlinear systems, 1350 Ph.D. Thesis, Kumamoto University.

1351

1352

1353

1354

1355

1356

1357

1358

1359

1360

1361

1362

1363 1364 1365

Chiu SL. 1994. Fuzzy Model Identification Based on Cluster Estimation. Journal of Intelligent \& Fuzzy Systems 2(3):267-278.

Cohn D, Ghahramani S, Jordan M. 1997. Active learning with mixture models. In: Murray-Smith R, Johansen T, editors. Multiple Model Approaches to Modeling and Control. London: Taylor and Francis. 360 p.

Dechnik-Vázquez YA, García-Barrios L, Ramirez-Marcial N, van Noordwijk M \& AlayonGamboa A. 2019. Assessment of browsed plants in a sub-tropical forest frontier by means of fuzzy inference. Journal of Environmental Management 236:163-181.

Dernoncourt F. 2013. Introduction to fuzzy logic. USA: Massachusetts Institute of Technology.

De Robertis A, Williams K. 2008. Weight-length relationships in fisheries studies The standard allometric model should be applied with caution. Transactions of the American Fisheries Society 137(3):707-719.

Duan N. 1983. Smearing estimate: A nonparametric retransformation method. J Am Stat Assoc 78:605-610.

Eberhard WG. 2009. Static allometry and animal genitalia. Evolution 63:48-66. 
1366 Echavarría-Heras H, Leal-Ramírez C, Castro-Rodríguez JR, Villa-Diharce E, Castillo O. 2018. A

1367

1368

1369

1370

1371

1372

1373

1374

1375

1376

1377

1378

1379

1380

1381

1382

1383

1384

1385

1386

1387

1388

Takagi-Sugeno-Kang Fuzzy Model Formalization of Eelgrass Leaf Biomass Allometry with Application to the Estimation of Average Biomass of Leaves in Shoots: Comparing the Reproducibility Strength of the Present Fuzzy and Related Crisp Proxies. In: Castillo O, Melin P, Kacprzyk J, editors. Fuzzy Logic Augmentation of Neural and Optimization Algorithms. USA: Springer. p. 329-362.

Echavarría-Heras HA, Leal-Ramírez C, Villa-Diharce E, Cazarez-Castro NR. 2018. On the suitability of an allometric proxy for nondestructive estimation of average leaf dry weight in eelgrass shoots I: sensitivity analysis and examination of the influences of data quality, analysis method, and sample size on precision. Theoretical Biology and Medical Modelling 15(4):1-20.

Echavarría-Heras H, Leal-Ramírez C, Villa-Diharce E and Montesinos-López A. 2019. Examination of the Effects of Curvature in Geometrical Space on Accuracy of Scaling Derived Projections of Plant Biomass Units: Applications to the Assessment of Average Leaf Biomass in Eelgrass Shoots. BioMed Research International 1-23.

Ertel JE, Fowlkes EB. 1976. Some algorithms for linear spline and piecewise multiple linear regression. Journal of the American Statistical Association 71:640-648.

Feng C, Wang H, Lu N, Chen T, He H, Lu Y, Tu XM. 2014. Log-transformation and its implications for data analysis. Shanghai Archives of Psychiatry 26(2).

Forbes TL, López GR. 1989. Determination of critical periods in ontogenetic trajectories. Functional Ecology 3:625-632.

Frankino WA, Emlen DJ, Shingleton AW. 2010. Experimental approaches to studying the evolution of animal form: the shape of things to come. Pages 419-478 in Experimental 
1389

1390

1391

1392

1393

1394

1395

1396

1397

1398

1399

1400

1401

1402

1403

1404

1405

1406

1407

1408

1409

1410

Evolution: Concepts, Methods, and Applications of Selection Experiments, edited by T. Garland Jr. and Rose MR. Berkeley (CA): University of California Press.

Gan MT, Hanmandlu M, Tan AH. 2005. From Gaussian mixture model to additive fuzzy systems. IEEE Transactions on Fuzzy Systems 13(3):303-316.

Gerber S, Eble GJ, Neige P. 2008. Allometric space and allometric disparity: a developmental perspective in the macroevolutionary analysis of morphological disparity. Evolution 62$6: 1450-1457$.

Gingerich PD. 2000. Arithmetic or geometric normality of biological variation: an empirical test of theory. Journal of Theoretical Biology 204:201-221.

Glazier D, Powell M, Deptola T. 2013. Body-size scaling of metabolic rate in the trilobite Eldredgeops rana. Paleobiology 39(1): 109-122.

Glazier DS. 2013. Log-transformation is useful for examining proportional relationships in allometric scaling. Journal of Theoretical Biology 334:200-203.

Gould SJ. 1966. Allometry and size in ontogeny and phylogeny. Biological Reviews 41:587-640.

Gupta HV, Sorooshian S, Yapo PO. 1998. Toward improved calibration of hydrologic models: Multiple and noncommensurable measures of information. Water Resources Research 751-763.

Hamilton F, Lloyd AL, Flores KB. 2017. Hybrid modeling and prediction of dynamical systems. PLoS Comput Biol 13(7).

Harris LA, Duarte CM, Nixon SW. 2006. Allometric laws and prediction in estuarine and coastal ecology. Estuaries and Coasts 29:343-347.

Hartnoll R. 1978. The determination of relative growth in Crustacea. Crustaceana 34:281-293. 
1411 Hauduc H, Neumann MB, Muschalla D, Gamerith V, Gillot S Vanrolleghem PA. 2011. Towards

1412

1413

1414

1415

1416

1417

1418

1419

1420

1421

1422

1423

1424

1425

1426

1427

1428

1429

1430

1431

1432

quantitative quality criteria to evaluate simulation results in wastewater treatment $-\mathrm{A}$ critical review. Proceedings 8th symposium on systems analysis and integrated assessment.

Herrera-Viedma E, López-Herrera AG. 2010. A review on information accessing systems based on fuzzy linguistic modelling. International Journal of Computational Intelligence Systems 3(4): 420-437.

Hills M. 2013. Allometry. In: Balakrishnan N, editor. Methods and applications of statistics in the atmospheric and Earth Sciences. United States: Willey and Sons, Inc. 329-362.

Hood G. 2007. Landscape allometry and prediction in estuarine ecology: Linking landform scaling to ecological patterns and processes. Estuaries and Coasts 30:895-900.

Houle D, Pélabon C, Wagner G, Hansen T. 2011 Measurement and meaning in biology. Q. Rev. Biol. 86:3-34.

Huxley JS. 1924. Constant differential growth-ratios and their significance. Nature 114: 895-896.

Huxley JS. 1927. Further work on heterogonic growth. Biol. Zent. Bl. 47: 151-163.

Huxley JS. 1932. Problems of relative growth. London UK: Methuen.

Ibrahim D. 2016. An Overview of Soft Computing, Procedia Computer Science 102: 34-38.

Jang. J.S.R ANFIS: Adaptive-Network-based Fuzzy Inference Systems. IEEE Transactions on Systems, Man, and Cybernetics, 23(03):665-685, May 1993

Jang JS, Sun CT, Mizutani E. 1997. Neuro-Fuzzy and Soft Computing: A computational approach to learning and machine intelligence. Upper Saddle River, NJ: Prentice-Hall.

Johansen TA, Foss BA. 1997. Operating regime based process modeling and identification. Computers \& Chemical Engineering 21(2):159-176. 
1433 Julious SA. 2001. Inference and estimation in a changepoint regression problem. Statistician $1434 \quad 50: 51-61$.

1435 Kalbfleisch JG. 1985. Probability and statistical inference, statistical inference, 2 edition. Berlin: $1436 \quad$ Springer Berlin.

1437 Kawaji S. 2002. Hybrid Soft Computing Approaches to Identification of Nonlinear Systems, IFAC $1438 \quad$ Proceedings 35(1): 187-192.

1439 Kerkhoff AJ, Enquist BJ. 2009. Multiplicative by nature: why logarithmic transformation is 1440 necessary in allometry. Journal of Theoretical Biology 257:519-521.

1441 Kimmins JP, Mailly D, Seely B. 1999. Modelling forest ecosystem net primary production: the 1442 hybrid simulation approach used in forecast, Ecological Modelling, 122(3): 195-224.

1443 Klingenberg PC. 1998. Heterochrony and allometry: the analysis of evolutionary change in 1444 ontogeny. Biol Rev 73: 79-123.

1445 Klingenberg CP. 2016. Size, shape, and form: concepts of allometry in geometric morphometric 1446 Dev Genes Evol; 226(3):113-37.

1447 Knell RJ, Pomfret JC, Tomkins JL. 2004. The limits of elaboration: curved allometries reveal the 1448 constraints on mandible size in stag beetles. Proceedings of the Royal Society of London B 1449 $271: 523-528$.

1450 Koch RW, Smillie GM. 1986. Comment on "River loads underestimated by rating curves" by R.I. 1451 Ferguson. Water Resource Research 22: 2121-2122.

1452 Kolokotrones, T, Savage V. Deeds EJ, Fontana W. 2010. Curvature in metabolic scaling. Nature $1453 \quad$ 464: 753-6.

1454 Lai J, Yang B, Lin D, Kerkhoff AJ, Ma. K. 2013. The Allometry of Coarse Root Biomass: Log1455 Transformed Linear Regression or Nonlinear Regression? PLoS ONE. 
1456 Larsen PM. 1980. Industrial applications of fuzzy logic control. International Journal of Man1457 Machine Studies 12:3-10.

1458 Lemaître JF, Vanpé C, Plard F, Gaillard JM. 2014 The allometry between secondary sexual traits 1459 and body size is nonlinear among cervids. Biol. Lett. 10: 20130869.

1460 Lemaître JF, Vanpé C, Plard F, Pélabon C, Gaillard JM. 2015. Response to Packard: make sure we do not throw out the biological baby with the statistical bath water when performing allometric analyses. Biol Lett

1463

1464

1465

1466

1467

1468

1469

1470

1471

1472

1473

1474

1475

1476

1477

Lin LIK. 1989. A concordance correlation coefficient to evaluate reproducibility. Biometrics $45: 255-268$.

Lovett D, Felder DL. 1989. Application of regression techniques to studies of relative growth in crustaceans. Journal of Crustacean Biology 9(Supp1. 4): 529-539.

McBride GB. 2005. A Proposal for strength-of-agreement criteria for Lin's Concordance Correlation Coefficient. NIWA Client Report: HAM2005-062. Hamilton, New Zeeland: National Institute of Water \& Atmospheric Research.

MacLeod CD. 2014. Exploring and Explaining Complex Allometric Relationships: A Case Study on Amniote Testes Mass Allometry. Systems 2:379-392.

MacLeod CD. 2010. Assessing the shape and topology of allometric relationships with body mass: a case study using testes mass allometry. Methods Ecol Evol 1:359 - 370.

Mamdani EH. 1977. Application of fuzzy logic to approximate reasoning using linguistic systems. IEEE Transactions on Computers C-26(12):1182-1191.

Manning WG. 1998. The logged dependent variable, heteroscedasticity and the retransformation problem. Health Economics 17(3):283-95. 
1478 Marquet PA, Quiñones RA, Abades S, Labra F, Tognelli M. 2005. Scaling and power-laws in 1479 ecological systems. Journal of Experimental Biology 208:1749-1769.

1480 Mascaro J, Litton CM, Hughes RF, Uowolo A, Schnitzer SA. 2014. Is logarithmic transformation 1481 necessary in allometry? Ten, one-hundred, one-thousand-times yes. Biological Journal of 1482 the Linnean Society 111:230-233.

1483 Mascaro J, Litton CM, Hughes RF, Uowolo A, Schnitzer SA. 2011. Minimizing Bias in Biomass 1484 Allometry: Model Selection and Log-transformation of Data. Biotropica 43(6):649-653.

1485 Mendel M. 2001. UNCERTAIN Rule-Based Fuzzy Logic Systems, Introduction and New 1486 Directions. USA: Prentice Hall.

1487 Meyer HA. 1938. The standard error of estimate of tree volume from logarithmic volume equation. 1488 J. For. 36: 340-342.

1489 Muggeo VM. 2003. Estimating regression models with unknown break-points. Statist. Med. 22: $1490 \quad 3055-3071$.

1491 Mosimann JE. 1970. Size allometry: size and shape variables with characterizations of the 1492 lognormal and generalized gamma distributions. J Am Stat Assoc 65:930-945.

1493 Näther W, Wälder K. 2006. Applying Fuzzy Measures for Considering Interaction Effects in Fine $1494 \quad$ Root Dispersal Models. Advances in Soft Computing 6: 373-381.

1495 Newman MC. 1993. Regression analysis of log-transformed data: Statistical bias and its $1496 \quad$ correction. Environmental Toxicology 12:1129-1133.

1497 Newman MEJ. 2007. Power laws, Pareto distributions and Zipf's law. Contemporary Physics 1498 $46: 323-351$. 
1499 Nevill AM, Bate S,Holder RL. 2005. Modeling physiological and anthropometric variables known 1500 to vary with body size and other confounding variables. Yearb Phys Anthropol 48:1411501

1502 Niklas KJ. Hammond ST. 2014. Assessing scaling relationships: uses, abuses, and alternatives. Int $1503 \quad$ J Plant Sci 175: 754-763.

1504 Oduguwa V, Tiwari A, Roy R. 2005. Evolutionary computing in manufacturing industry: an 1505 overview of recent applications. Applied Soft Computing 5(3): 281-299.

1506

Packard GC, Birchard GF. 2008. Traditional allometric analysis fails to provide a valid predictive 1507 model for mammalian metabolic rates. Journal of Experimental Biology 211:3581-7.

1508 Packard GC, Boardman TJ. 2008. Model selection and logarithmic transformation in allometric 1509 analysis. Physiological and Biochemical Zoology 81:496-507.

1510 Packard GC. 2009. On the use of logarithmic transformations in allometric analyses. Journal of $1511 \quad$ Theoretical Biology 257:515-518.

1512 Packard GC. 2012a. Julian Huxley, Uca pugnax and the allometric method. Journal of 1513 Experimental Biology, 215: 569-573;

1514 Packard GC. 2012b. Is non-loglinear allometry a statistical artifact?. Biological Journal of the 1515 Linnaean Society 107(4):764-773.

1516 Packard GC. 2013. Is logarithmic transformation necessary in allometry? Biological Journal of 1517 the Linnean Society 109:476-486.

1518 Packard GC. 2016. Relative growth by the elongated jaws of gars: A perspective on polyphasic 1519 loglinear allometry. Journal of Experimental Zoology (Molecular and Developmental 1520 Evolution) 326B:168-175. 
1521 Packard GC. 2017a. The essential role for graphs in allometric analysis. Biological Journal of the $1522 \quad$ Linnaean Society 120:468-473.

1523 Packard GC. 2017b. Misconceptions about logarithmic transformation and the traditional 1524 allometric method. Zoology 123:115-120.

1525 Parresol B.R. 1999. Assessing tree and stand biomass: A review with examples and, critical 1526 comparisons. For. Sci. 45(4): 573-593.

1527 Pélabon C, Firmat C, Bolstad GH, Voje KL, Houle D, Cassara J, Rouzic AL, Hansen TF. 2014. 1528 Evolution of morphological allometry. Ann. N.Y. Acad. Sci. 1320: 58-75.

1529 Pélabon C, Tidière M, Lemaître JF, Gaillard JM. 2018. Modelling allometry: statistical and 1530 1531 biological considerations - a reply to Packard, Biological Journal of the Linnean Society 125(3): 664-671.

Pozna C, Precup RE, Tar JK, Škrjanc I, Preitl S. 2010. New results in modelling derived from Bayesian filtering. Knowledge-Based Systems 23(2): 182-194.

1534

Ramírez-Ramírez G, Ramírez-Avilés L, Solorio-Sánchez FJ, Navarro-Alberto JA, Dupuy-Rada 1535 JM. 2019. Shifts in tree allometry in a tropical dry forest: implications for above-ground 1536 biomass estimation, Botanical Sciences 97(2): 167-179.

1537 Raykov T, Marcoulides GA. 1999. On desirability of parsimony in structural equation model 1538 selection. Structural Equation Modeling, 6(3): 292-300.

1539 Rumlhart, D.E. Hinton, G. E and Williams R. J., Learning internal representations by error 1540 propagation. In D. E. Rumlhart and James L. McClelland, editors, Parallel distributed processing: volumen 1, chapter 8, pages 318-362. MIT Press, Cambridge, MA., 1986. 
1542 Schlaegen BE. 1982. Testing, reporting, and using biomass estimation models. P.95-112 in Proc.

1543 Of the $3^{\text {rd }}$ Annual Southern Forest Biomass Workshop, Gresham, C.A. (ed.) Belle W. 1544 Baruch For. Sci. Inst., Clemson Univ., Clemson SC. 137 p.

1545 Schreer 1997, Diving behavior of air-breathing vertebrates: allometry, classification, and 1546 interspecific comparisons. Ph.D. thesis, University of Waterloo, Ontario.

1547 Schwetter LRF, Bertone AMA. 2018. Fuzzy Modeling of the Weight - Length Allometric 1548 Relationship of the Fish Species Plagioscion Squamosissimus. International Journal for 1549 Innovation Education and Research 6(10): 306-313.

1550 Smith RJ. 1993. Logarithmic transformation bias in allometry. American Journal of Physical $1551 \quad$ Anthropology, 90(2):215-228.

1552 Strauss RE, Huxley JS. 1993. The study of allometry since Huxley. In: Thompson DH, editor. 1553 Problems of Relative Growth, new edition. Baltimore: Johns Hopkins University Press. p. $1554 \quad$ xlviii-lxxv.

1555 Sugeno M, Kang GT. 1988. Structure identification of fuzzy model. Fuzzy Sets and Systems 28:151556 33.

1557 1558

1559 1560 1561

1562 1563 1564

Takagi T, Sugeno M. 1985. Fuzzy identifications of systems and its applications to modeling and control. IEE Transactions on Systems, MAN and Cybernetics 15(1):116-132.

Tidière M, Lemaître JF, Pélabon C, Gimenez O, Gaillard JM. 2017. Evolutionary allometry reveals a shift in selection pressure on male horn size. Journal of Evolutionary Biology 30: 18261835

Tsuboi MW, van der B, Kopperud BT, Erritzøe J, Voje KL, Kotrschal A, Yopak KE, Collin SP, Iwaniuk AN, Kolm N. 2018. Breakdown of brain-body allometry and the encephalization of birds and mammals. Nat. Ecol. Evol. 2:1492-1500. 
1565 Vrkalovic E, Lunca C, Borlea ID. 2018. Model-Free Sliding Mode and Fuzzy Controllers for 1566 Reverse Osmosis Desalination Plants. International Journal of Artificial Intelligence $1567 \quad 16(2): 208-222$.

1568 Li X, Wang X, Zhang J, Wu L. 2015 Allometric scaling, size, distribution, and pattern formation 1569 of natural cities. Palgrave Communications 1:15017.

1570 Wang Li-Xin, Mendel JM. 1992. Fuzzy basis functions, universal approximation, and orthogonal 1571 least-squares learning. IEEE Transactions Neural Networks. 3(5):807-814.

1572 Weiner J. 2004. Allocation, plasticity and allometry in plants. Perspectives in Plant Ecology, $1573 \quad$ Evolution and Systematics 6: 207-215.

1574 West GB, Brown JH. 2005. The origin of allometric scaling laws in biology from genomes to 1575 ecosystems: towards a quantitative unifying theory of biological structure and 1576 organization. Journal of Experimental Biology 208:1575-1592.

1577 White EP, Xiao X, Isaac NJB, Sibly RM. 2012. Methodological tools. In Metabolic Ecology: A 1578 Scaling Approach; Sibly RM, Brown JH, Kodric-Brown A. Eds.; Wiley-Blackwell, 1579 Oxford, England; 2012; pp. 9-20. ISBN 9780470671535.

1580 Xiao X, White EP, Hooten MB, Durham SL. 2011. On the use of log-transformation vs. nonlinear 1581 regression for analyzing biological power laws. Ecology 92:1887-1894.

1582 Yager RR, Filev DP. 1994. Approximate clustering via the mountain method. IEEE Trans Syst 1583 Man Cybern 24:1279-1284.

1584 Ying H. 1998. General SISO Takagi-Sugeno fuzzy systems with linear rule consequent are 1585 universal approximators. IEEE Transactions on Fuzzy Systems 6(4):582-587.

1586 Zadeh LA. 1965. Fuzzy sets. Information and Control 8:338-353. 
1587 Zadeh LA. 1972. A Fuzzy-Set-Theoretic Interpretation of Linguistic Hedges. Journal of $1588 \quad$ Cybernetics 2(3):4-34.

1589 Zadeh LA. 1989. Knowledge representation in Fuzzy Logic. IEEE Transactions on Knowledge $1590 \quad$ Data Engineering 1:89-100.

1591 Zadeh LA. 1993. Fuzzy logic, neural networks and soft computing. One-page course 1592 announcement of CS 294-4. University of California at Berkeley.

1593 Zeng WS, Tang SZ. 2011. Bias correction in logarithmic regression and comparison with weighted 1594 regression for non-linear models. Nature Precedings.

1595 Zeng WS, Tang SZ. 2011. Goodness evaluation and precision analysis of tree biomass equations. $1596 \quad$ Scientia Silvae Sinicae 47:106-113.

1597 Zeng WS, Duo HR, Lei XD, Chen XY, Wang XJ, Pu Y, Zou WT. 2017. Individual tree biomass 1598 equations and growth models sensitive to climate variables for Larix spp. in China. $1599 \quad$ European Journal of Forest Research 136(2):233-249.

1600 Zeng K, Nai-Yao Z, Wen-Li X. 2000. A comparative study on sufficient conditions for Takagi1601 Sugeno fuzzy systems as universal approximators. IEEE Transactions on Fuzzy Systems $1602 \quad 8(6): 773-780$.

1603 


\section{Figure 1}

Spreads of allometric response and covariate in geometrical space.

This plot shows the spread response-covariate in geometrical space for the included data sets. Panel (a) depicts dispersion for Echavarría-Heras et al. (2019), panel (b) presents that associating to Mascaro et al. (2014) panel (c) shows that for Huxley (1932) and panel (d) is for the De Robertis \& Williams (2008) data sets. 
a)

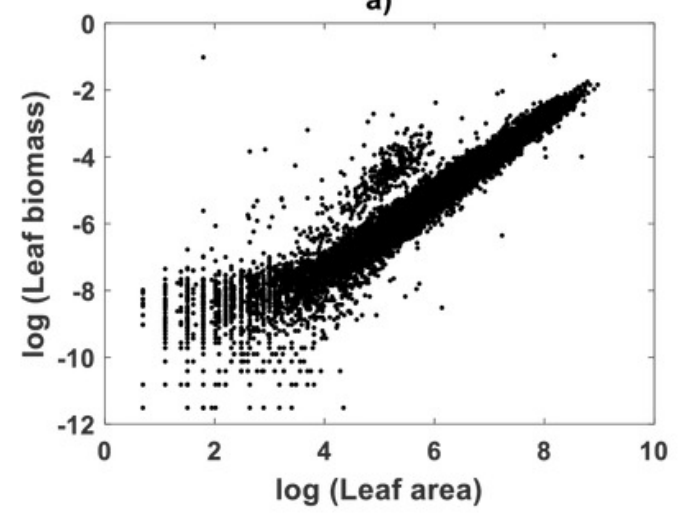

c)

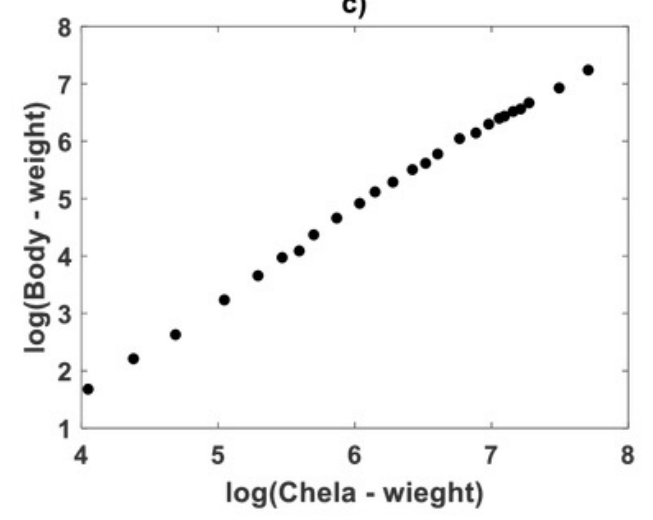

b)

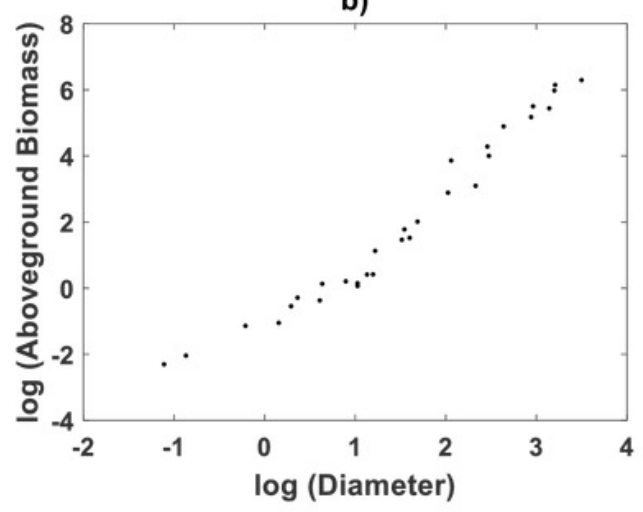

d)

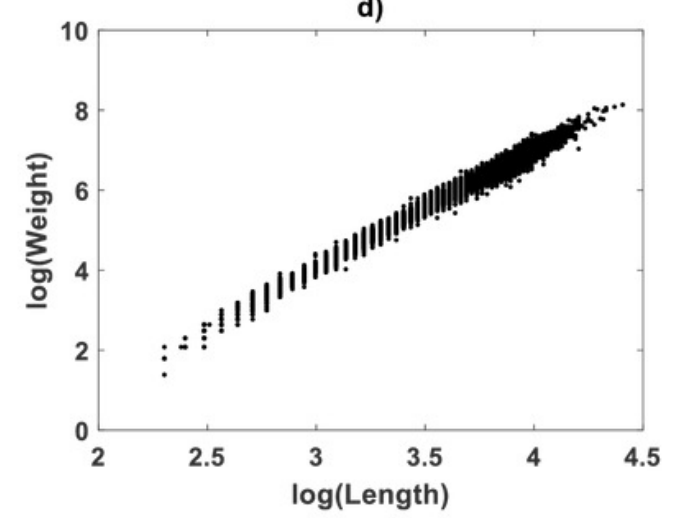




\section{Figure 2}

Dispersion plots for the TAMA fit on the Echavarría-Heras et al. (2019) data set.

Panel (a) shows dispersion of log transformed eelgrass leaf biomasses $v$ around the estimated form of mean response line of equation (11). Residuals for the regression model of equation (12) show irregular spreading about the zero line (panel (b)). Besides, the QQ plot in panel (c) displays heavier tails than expected for a normal distribution. 


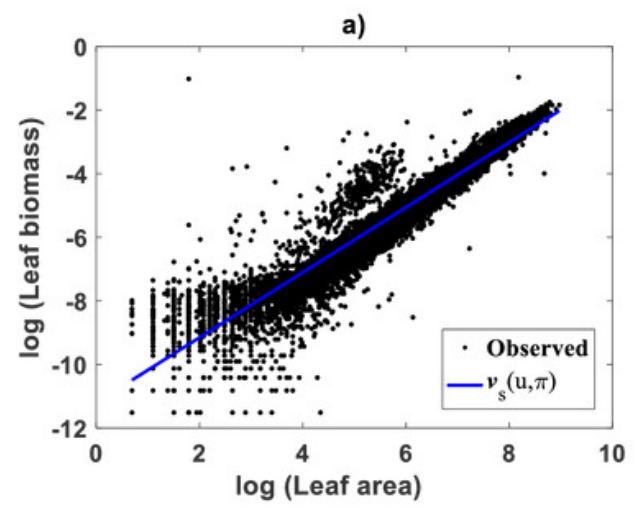

b)
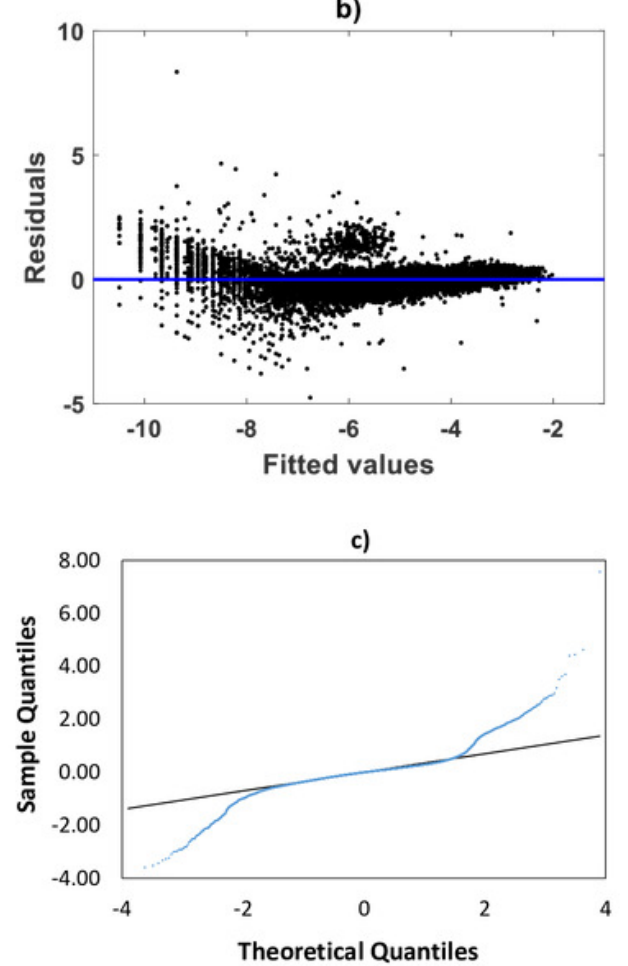


\section{Figure 3}

Elements of the TSK-PLA fuzzy model identified on the Echavarría-Heras et al. (2019) data set.

Shown results associate to a value $r_{a}=0.47$ for the clustering radius that corresponded to a $q=2$, heterogeneity index. Panel (a) displays plots of membership functions both given in the Gaussian form of equation (21). Panel (b) presents plots of normalized firing strength factors given by equations (31) and (32) one to one. A break point at $u_{b}=3.98$ is shown. Panel (c) displays consequent linear functions as given by equations (33) and (34). Panel (d) portraits component products. 

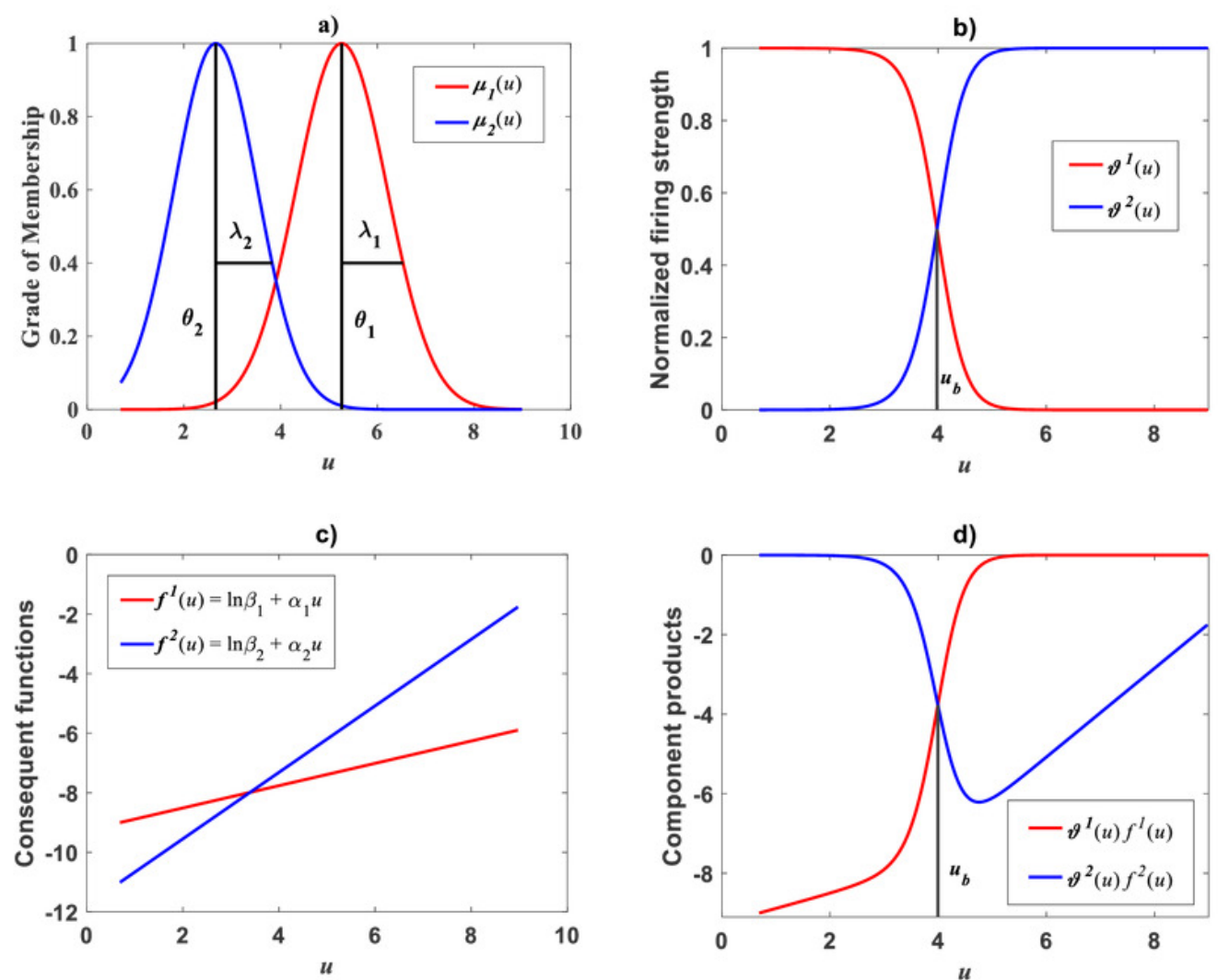


\section{Figure 4}

Fitting results of the TSK -PLA model for the Echavarría-Heras et al. (2018) data set.

Panel (a) shows the dispersion about the mean response curve identified through the regression model of equation (17) assuming heteroscedasticity in the form set by equation (35). Panel (b) displays residual spread about the zero line. Region bounded by red lines determine (95\%) confidence intervals. Panel (c) presents corresponding QQ plot. Opposing a biased spreading about the mean response in Fig. $2 \mathrm{a}$, distribution around the TSK-PLA mean response is fair all over the domain of the log transformed response. Shown breaking point separates two phases conforming the identified non-log linear allometry. 
a)

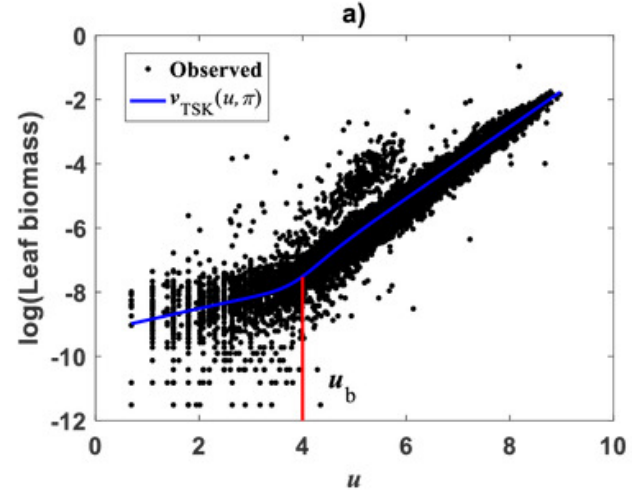

b)

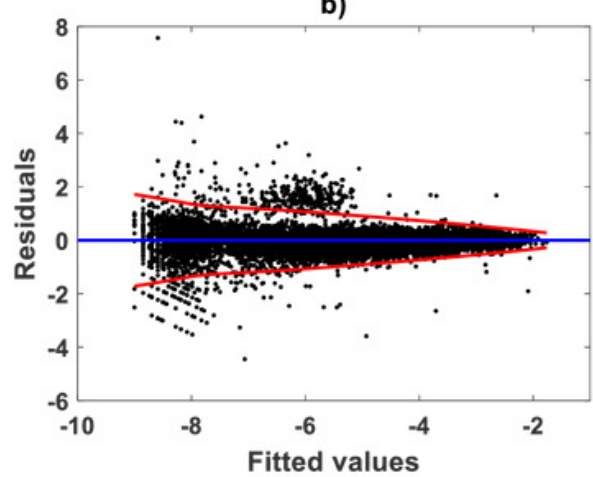

c)

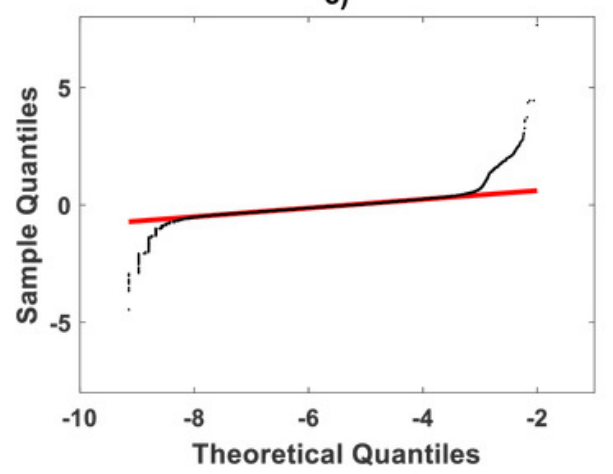




\section{Figure 5}

Comparison of TAMA and TSK-PLA mean responses in arithmetical scales fitted on the Echavarría-Heras et al. (2018) data set.

Panel (a) shows the distribution of observed eelgrass leaf biomass values $y$ about the mean response $E_{g т s k}(y \mid x)$ (cf. equation (19)). Equation (10) provided the form of the correction factor. Panel (b) shows the extent of bias tied to proxies $E_{g s}(y \mid x)$ calculated through the TAMA scheme and a Duan's form of the correction factor. Panel (c) exhibits a remarkable correspondence between $E_{a s}(y \mid x)$ derived from a fit of Huxley's formula of simple allometry and the asymptotic mean response derived from the TSK-PLA model (equation (47)). 

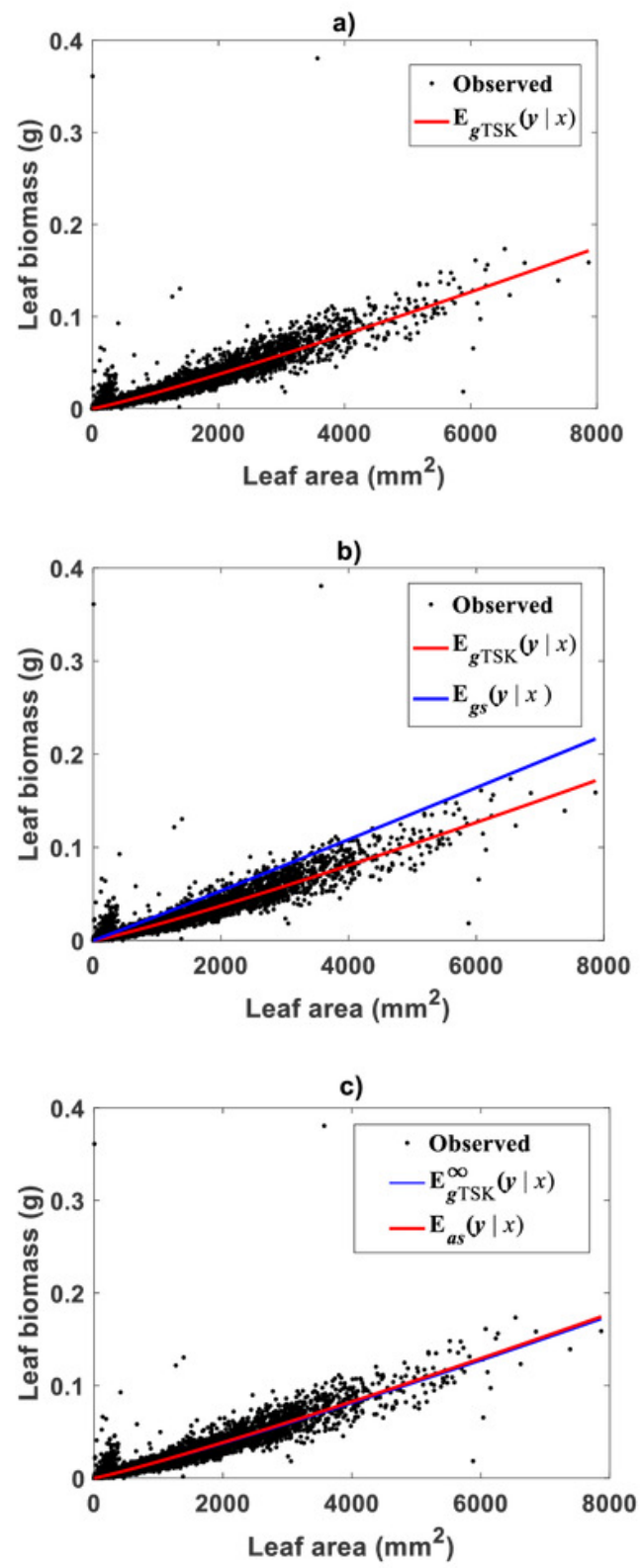


\section{Figure 6}

Elements of the TSK-PLA fuzzy model identified on the Mascaro et al. (2011) data set.

Shown results associated to a value $r_{a}=0.80$ for the clustering radius and corresponding to a $q=2$, heterogeneity index. Panel (a) plots of membership functions both given in the Gaussian form of equation (21). Panel (b) presents plots of normalized firing strength factors given by equations (31) and (32) one to one. A break point at $u_{b}=1.575$ is shown. Panel (c) displays consequent linear functions as given by equations (33) and (34). Panel (d) portraits component products. 

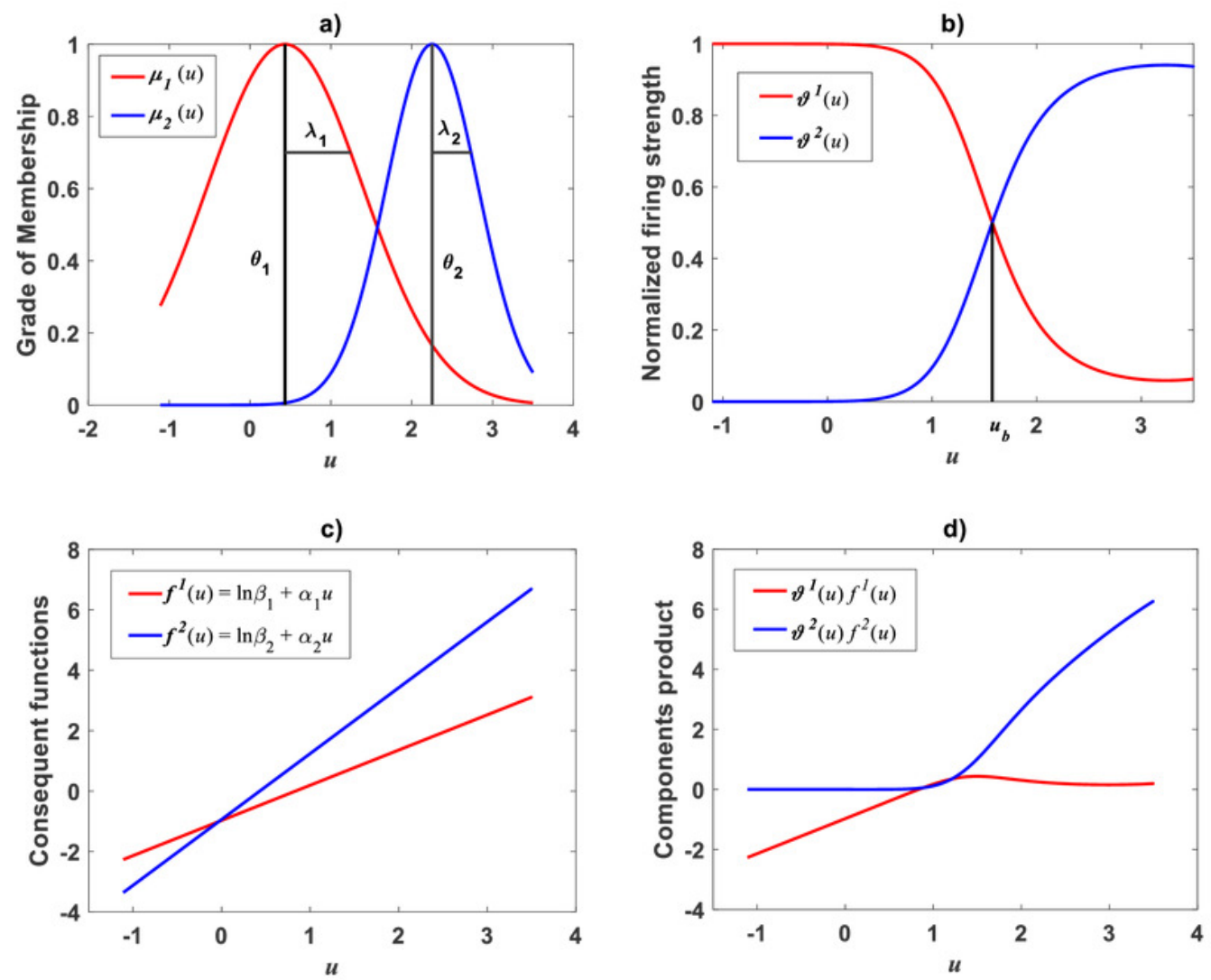


\section{Figure 7}

Spread plots for the TSK-PLA model fitted on the Mascaro et al. (2011) data set.

Spread about the TSK-PLA mean response displays remarkable reproducibility and consistency of biphasic allometry (panel (a)). Residual plot displays a fair spread about the zero line (panel (b)). Normal-QQ plot show a large plateau where residuals track a normal distribution pattern (panel (c)). Panels (d), (e) and (f) show spread about mean response, residual and QQ-plot of TAMA's fit to this data one to one. 
a)

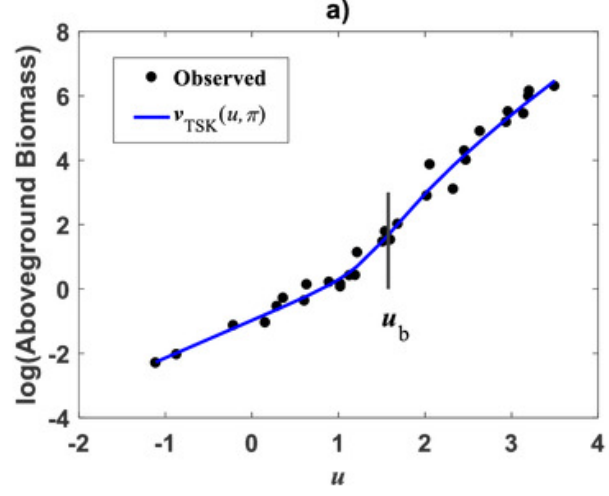

b)

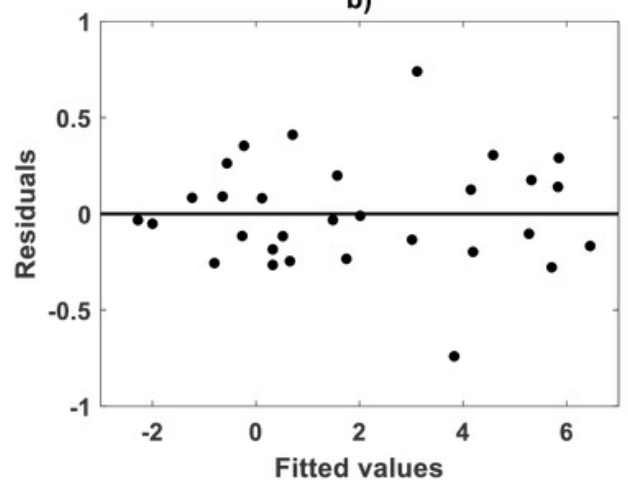

c)

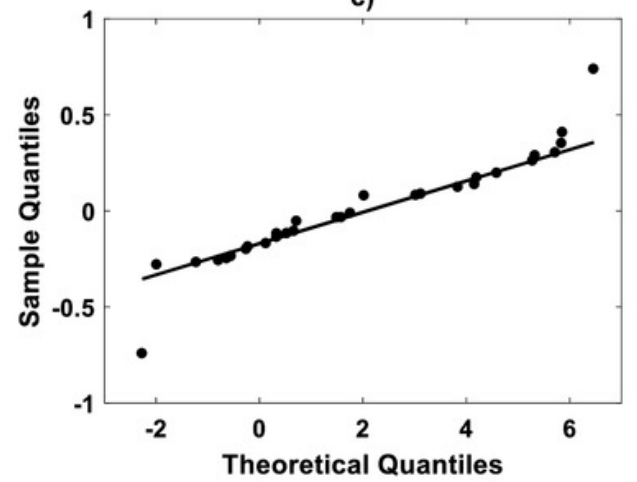

d)

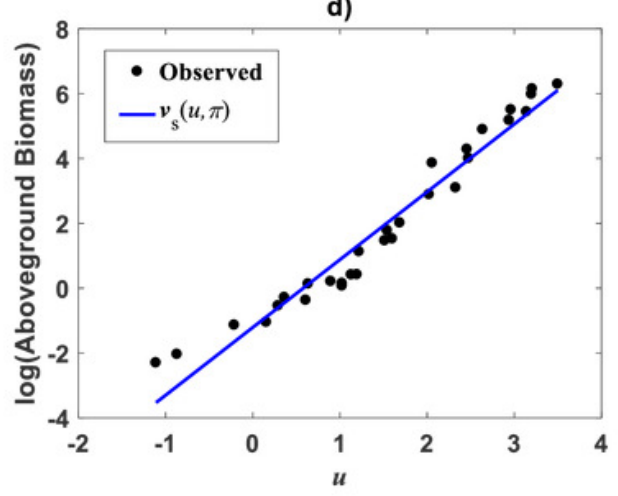

e)

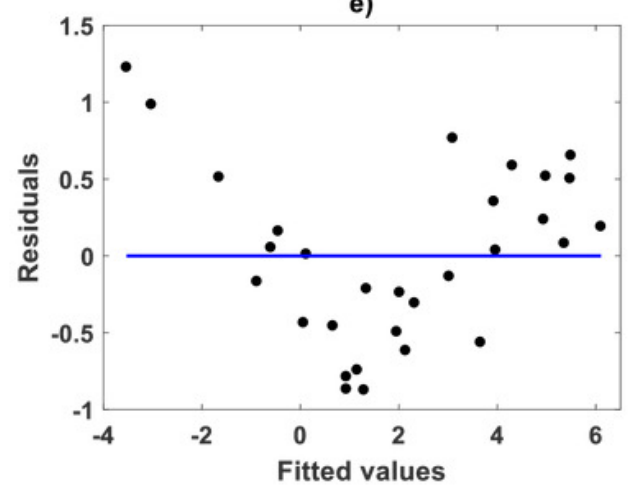

f)

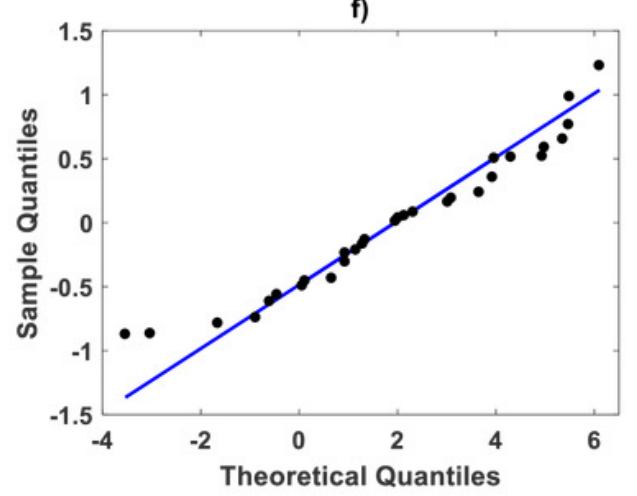




\section{Figure 8}

TSK-PLA model identified on Huxley (1932) Uca pugnax data.

For $r_{a}=0.8$ the fuzzy inference system returned $q=2$ heterogeneity. Panel (a) exhibits firing strengths intersecting at a break point $u_{b}=5.813$ in original log scales. Panel (b) acquired linear consequents. Panel (c) component products. Panel (d) shows position of $u_{b}$ relative to Huxley's break point $u_{b H}$ in a display conforming that in Fig. 3 of Huxley (1932). Panel (e) spread about TSK-PLA interpolation function produced by $r_{a}=0.2$ and $q=2$ in original $\log$ scales. This plot shows $u_{b 1}=6.78$ one of detected breakpoints. This can be considered as a proxy for Huxley's designated break point $u_{\text {ьн. }}$. Interpolation results in panel (e) suggest the biphasic arrangement of linear segments about $u_{b l}$ shown in panel (f). 
a)

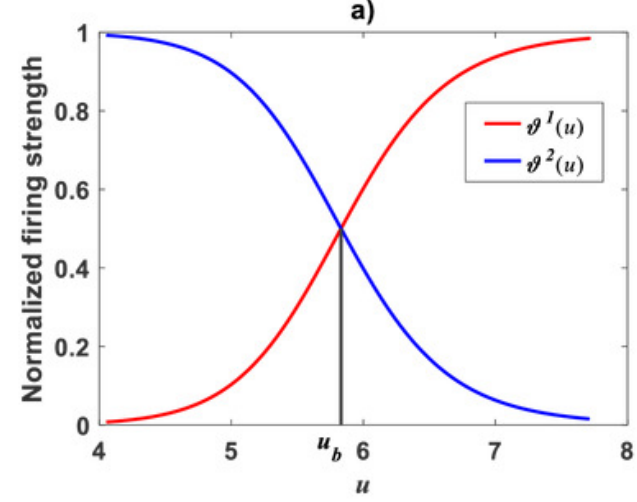

c)

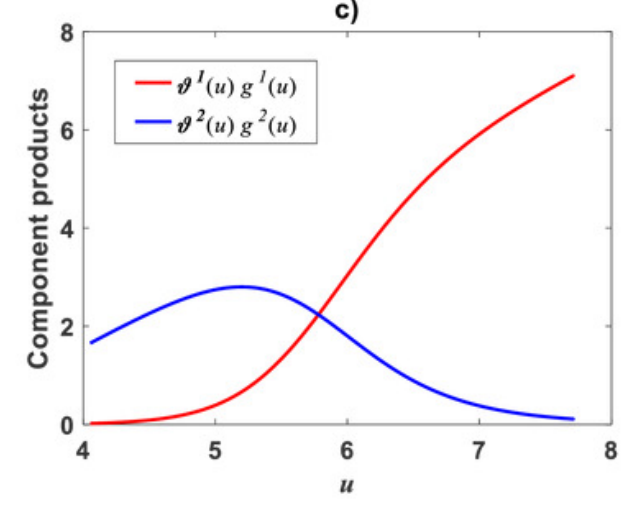

e)

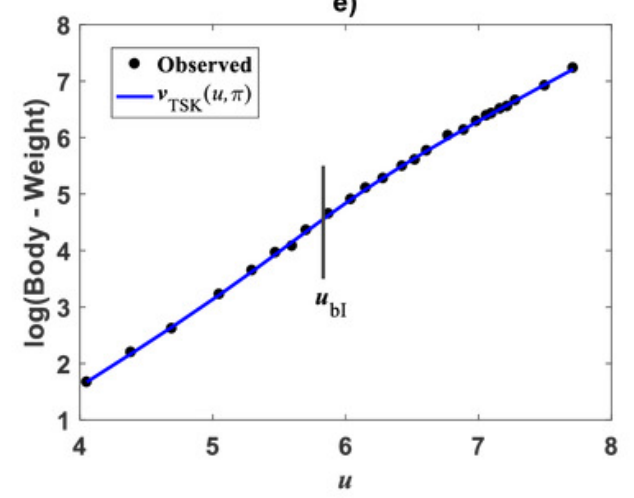

b)

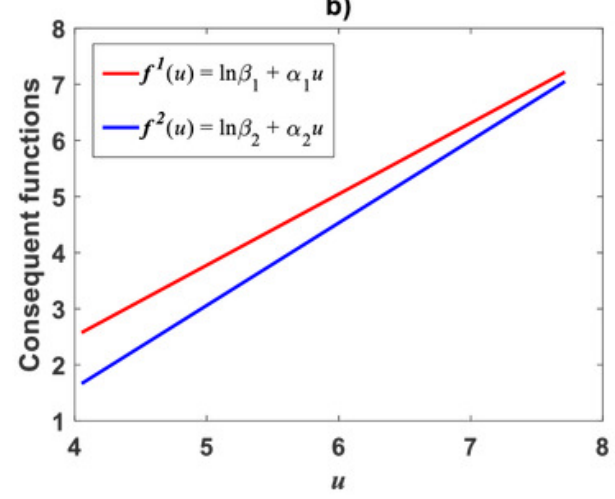

d)
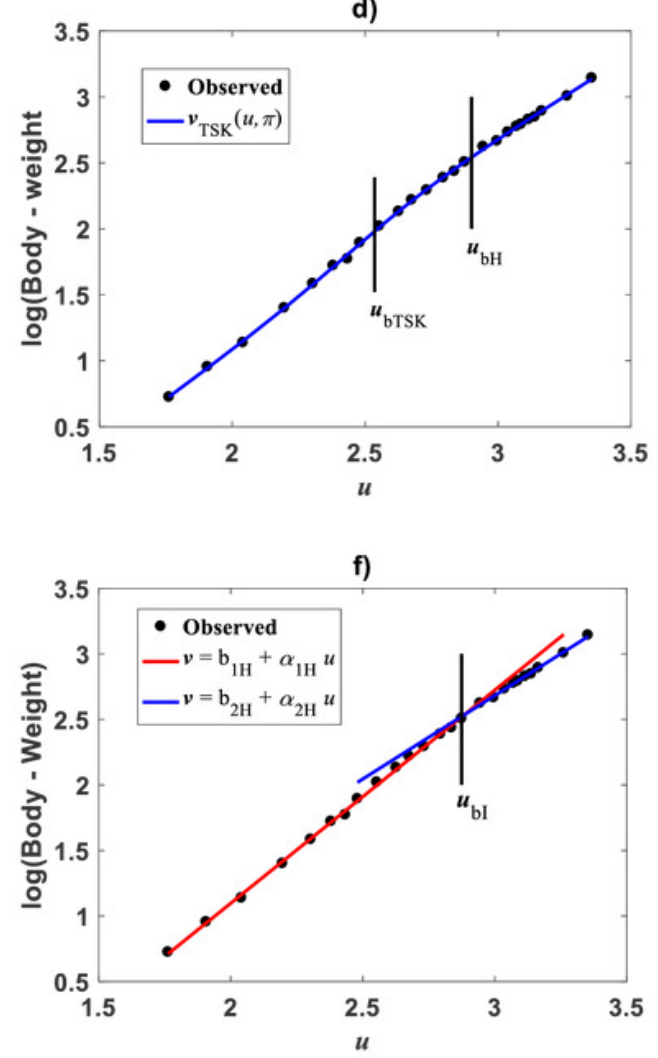
Figure 9

Comparison TAMA vs TSK-PLA fuzzy model fitted on Huxley (1932) Uca pugnax data.

Panel (a) exhibits spread about TSK-PLA mean response as determined by a $r_{a}=0.8$ and $q=$ 2 fit on Huxley's Uca pugnax data. Associating residual and QQ-plots are shown in panel (b) and panel (c) one to one. Panel (d) trough panel (f) display corresponding spreads produced by TAMA's fit to referred data. 
e)

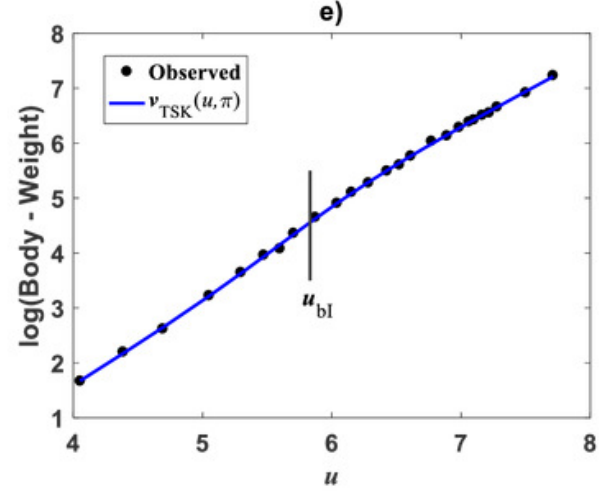

b)

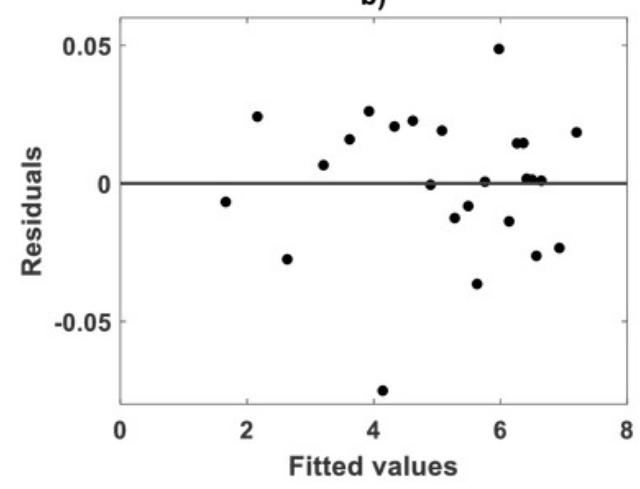

c)

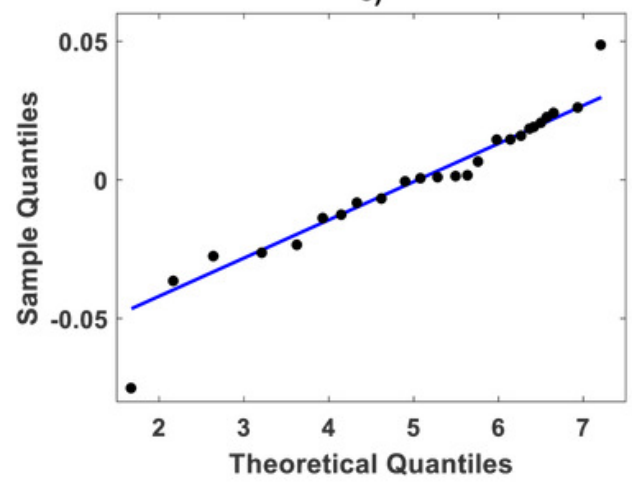

d)

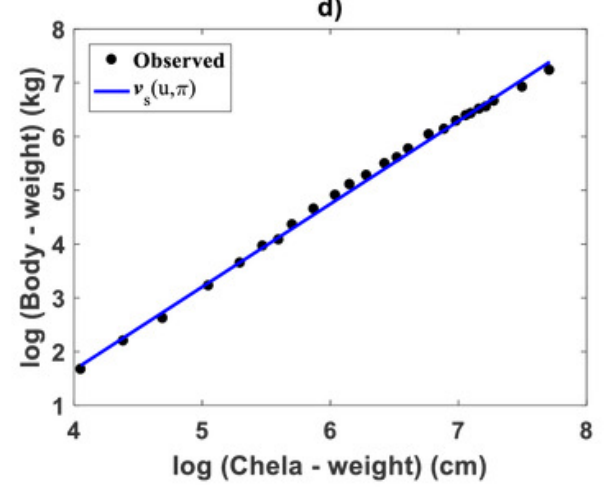

e)

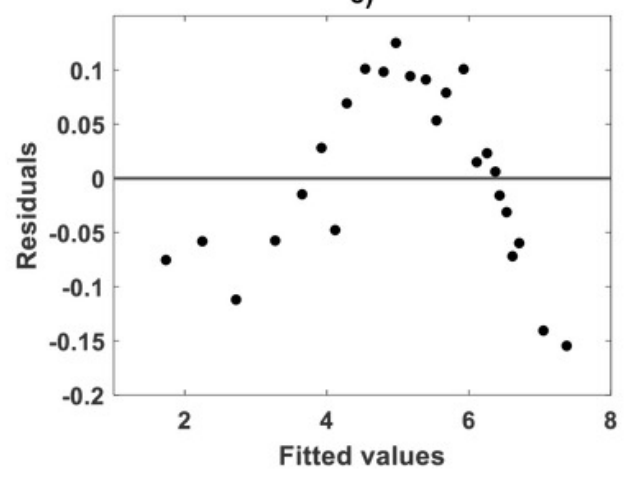

f)

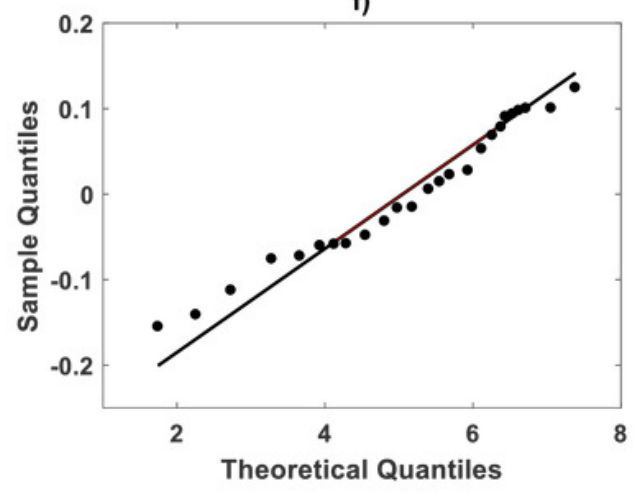




\section{Figure 10}

Elements of the TSK-PLA model identified on the De Robertis and Williams (2008) data.

A single membership function in the Gaussian form given by equation (21) is shown in panel (a). Corresponding firing strength is displayed in panel (b). Consequent function appears in panel (c). Component product appears in panel (d). These components rule out nonlinearity in geometrical space, suggesting consistency of a TAMA approach. 
a)

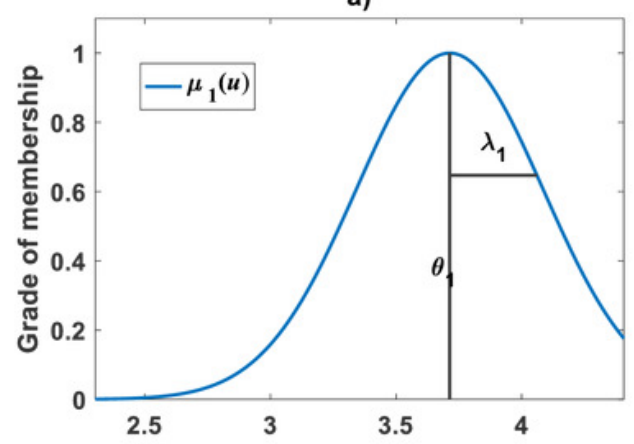

c)

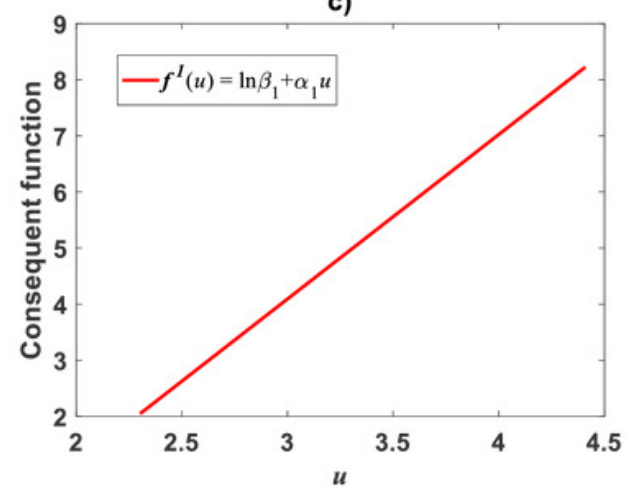

b)

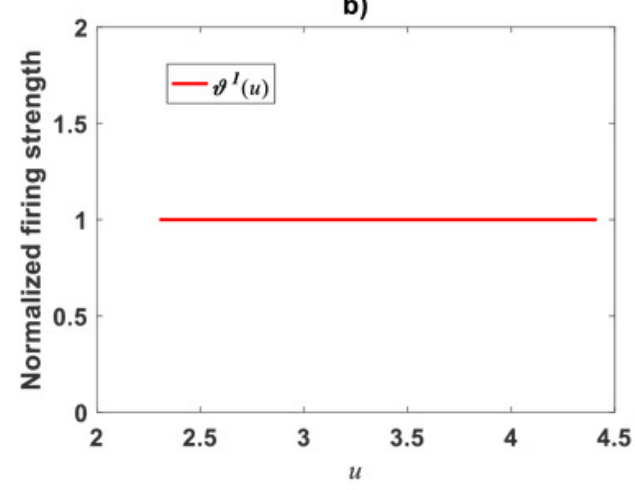

d)

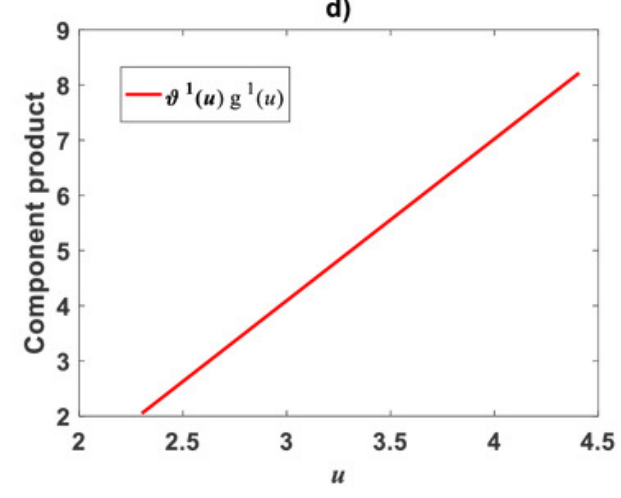


Figure 11

Comparison of TAMA and TSK-PLA fuzzy model fitted on the De Robertis and Williams (2008) data set.

Panels (a), (b) and (c) display spread about mean response, residual plot and QQ-Normal plot for the fit of the TAMA protocol one to one. Panels (d), (e) and (f) present corresponding plots for the fit of the TSK fuzzy model. 
a)

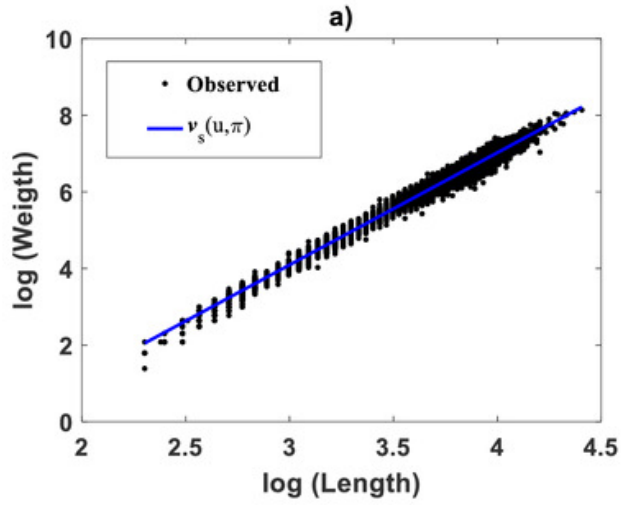

b)

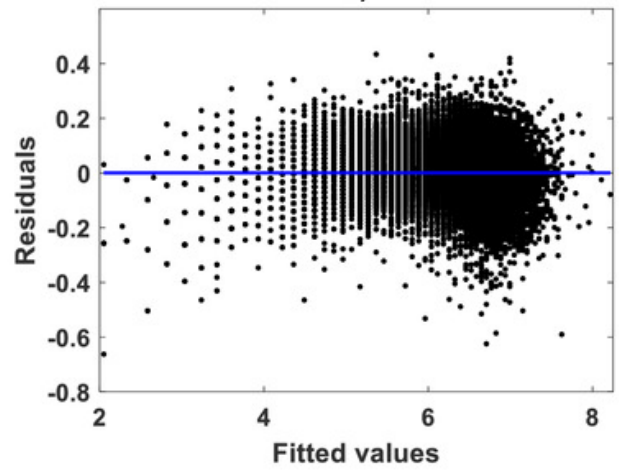

c)

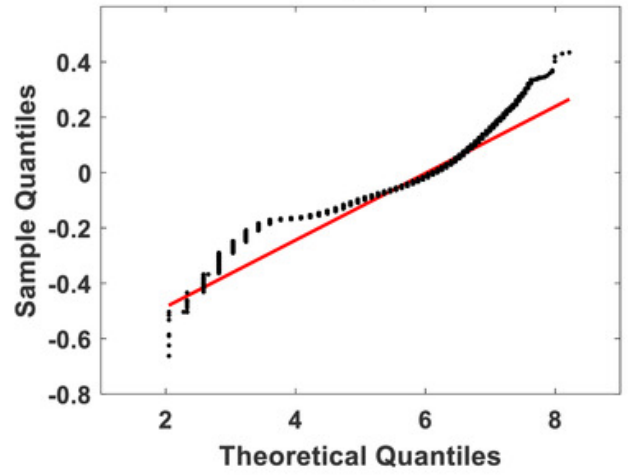

d)

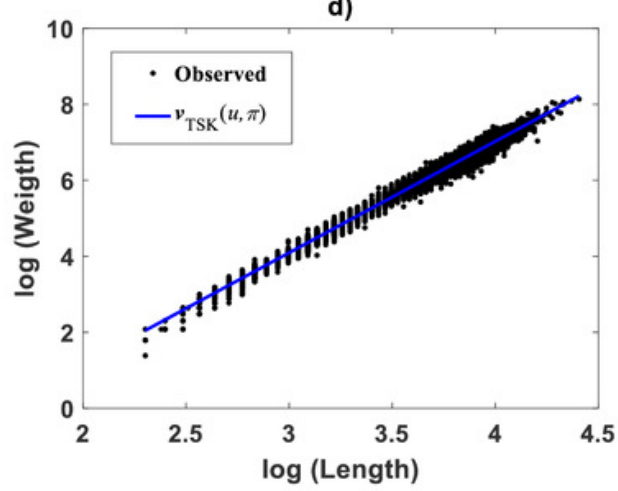

e)

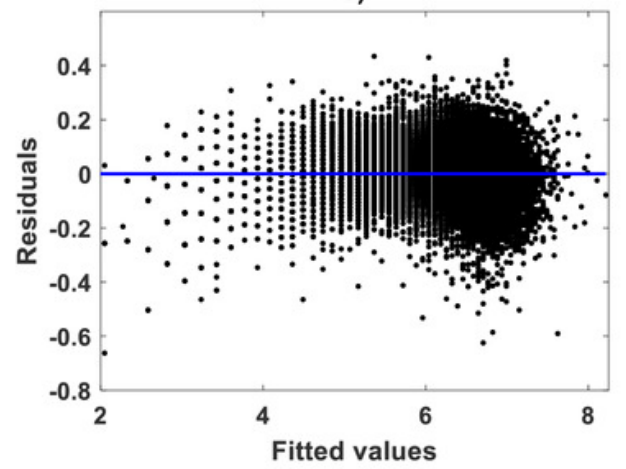

f)

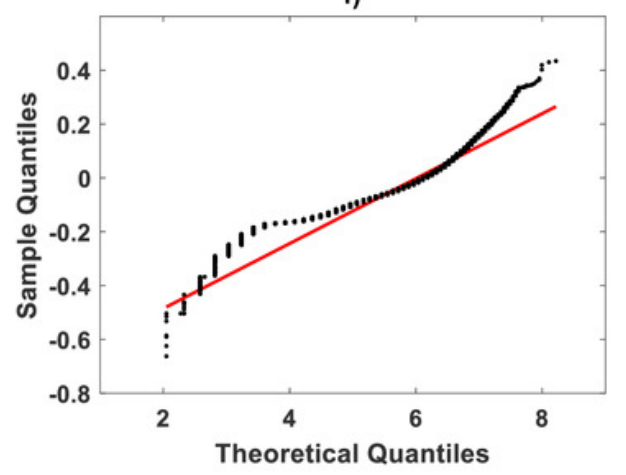




\section{Figure 12}

TSK-MPCA fitted on the Echavarría-Heras et al. (2019).

Setting $r_{a}=0.5416$ returned $q=2$ for present eelgrass data analyzed by means of the TSKMPCA fuzzy model of Eq. (48) through Eq. (53). Panel (a) firing strength factors detecting a break point placed at $x_{b}=49.632$. Panel (b) spread about fitted mean response function $E_{\text {aтк }}(y \mid x)$ compared to $E_{g \text { тรк }}(y \mid x)$ derived from retransformation of the TSK-PLA output. We can be aware that reproducibility strengths are equivalent. This can be stressed by performance metrics in Table 8. Panel (c) trough panel (d) presenting residual and QQ-plots confirm equivalence of $E_{\text {aтsk }}(y \mid x)$ and $E_{g \text { Tsk }}(y \mid x)$. 
a)

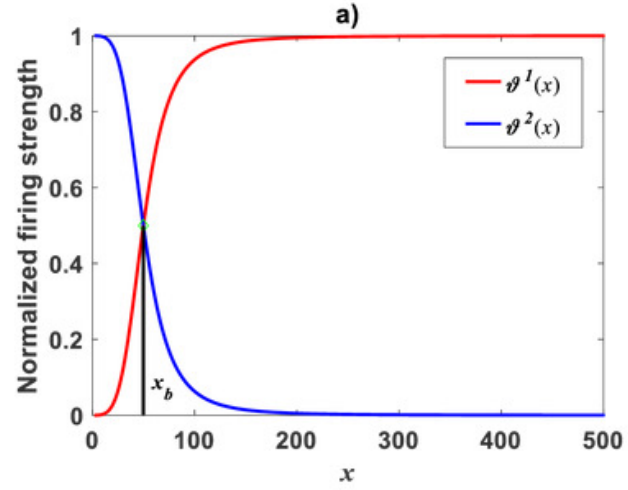

c)

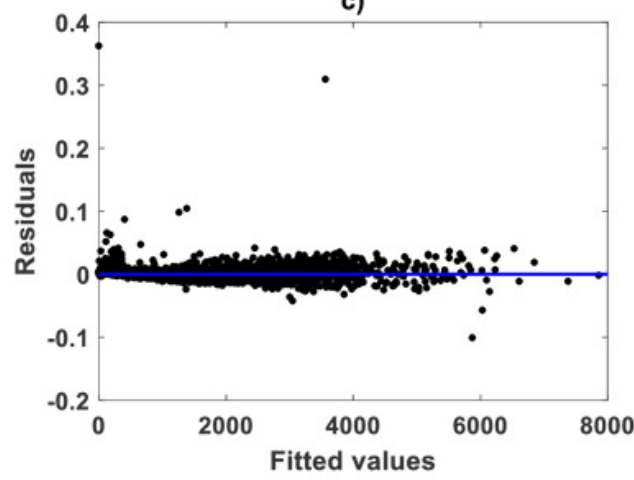

e)

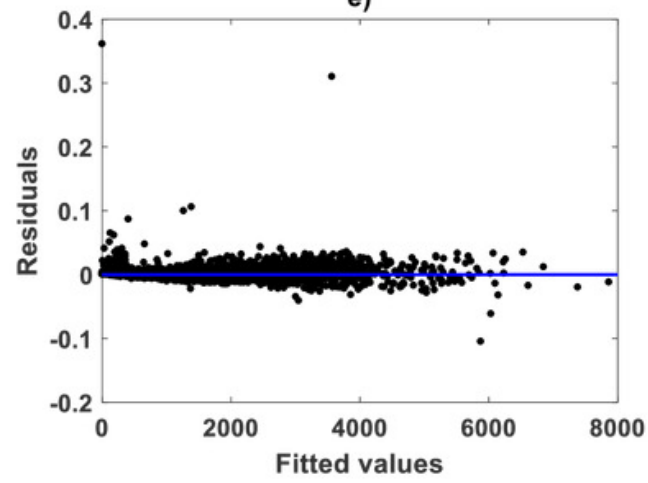

b)

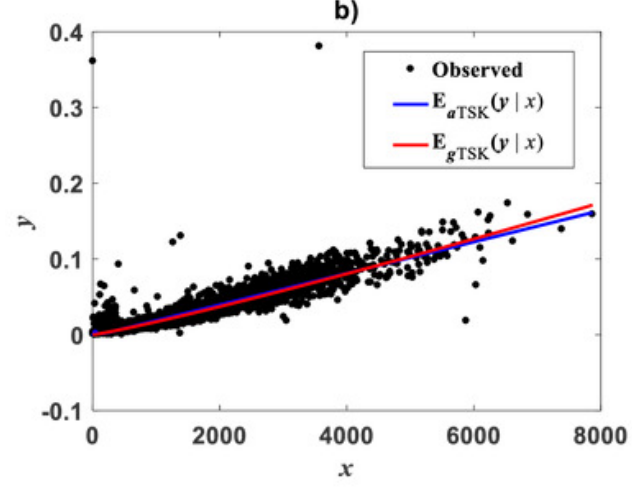

d)

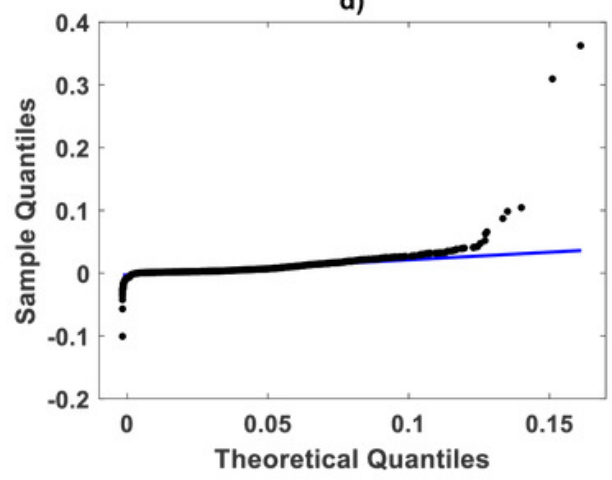

f)

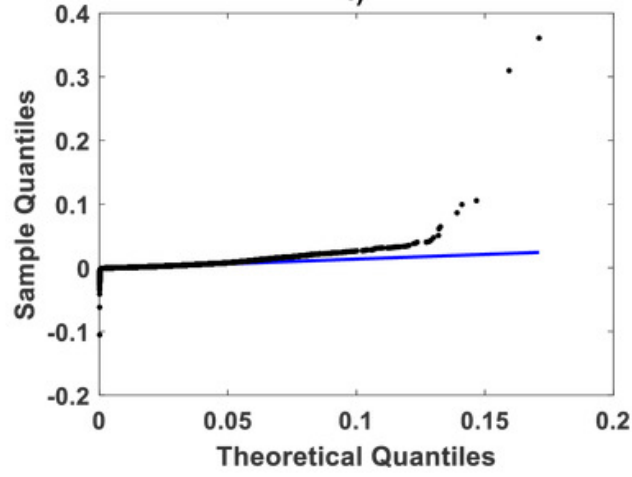




\section{Figure 13}

TSK-MPCA fitted on Mascaro et al. (2011) data set.

Panel (a) displays the $q=2$ firing strength factors deriving from a $r_{a}=0.855$ of the TSK-MPCA fit. We can learn of a break point estimated at $x_{b}=8.8662$. Spreads about fitted mean

functions shown in Fig. 13b reveal remarkable correspondence of projections by $E_{\text {aTsk }}(y \mid x)$ and $E_{g \text { TтK }}(y \mid x)$. This can be stressed by performance metrics in Table 9. Panel (c) and panel (d) residual and QQ plots for TSK-MPCA fit one to one. Equivalent plots for retransformed results of TSK- PLA fitted on this data are displayed in panel (e) and panel (f). 
a)

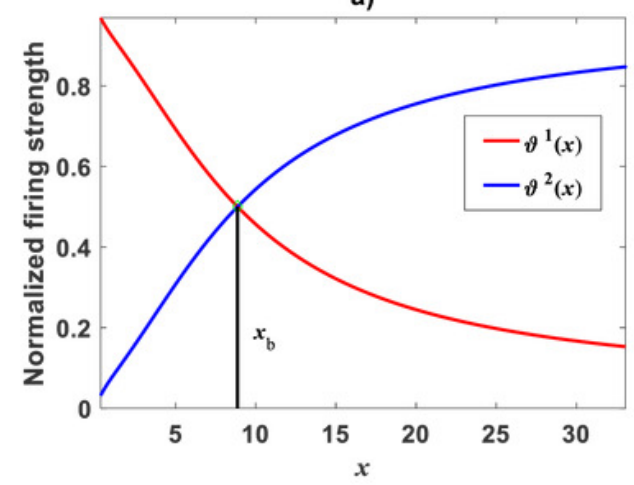

c)

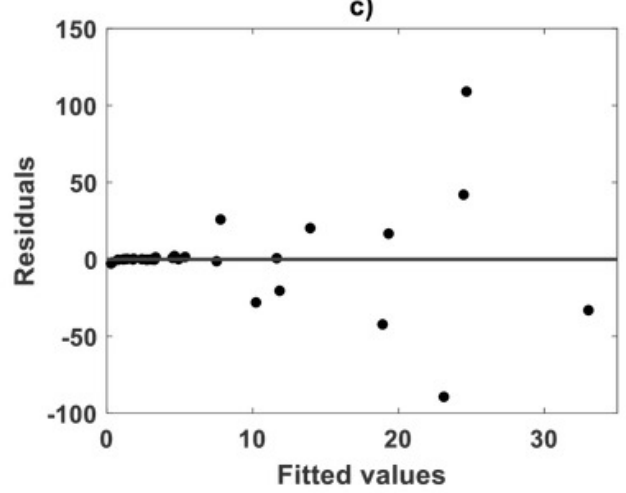

e)

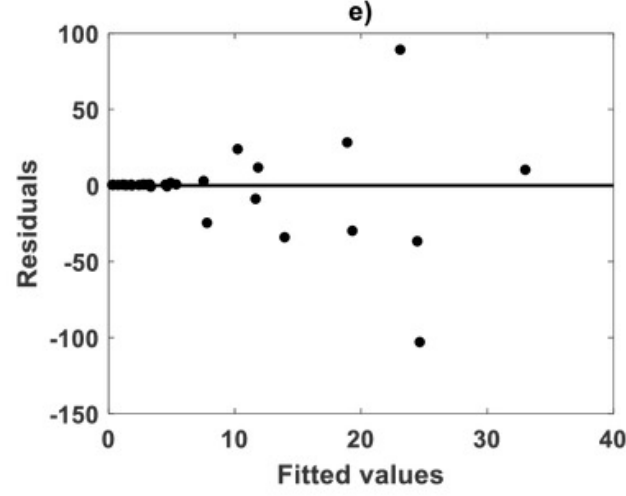

b)

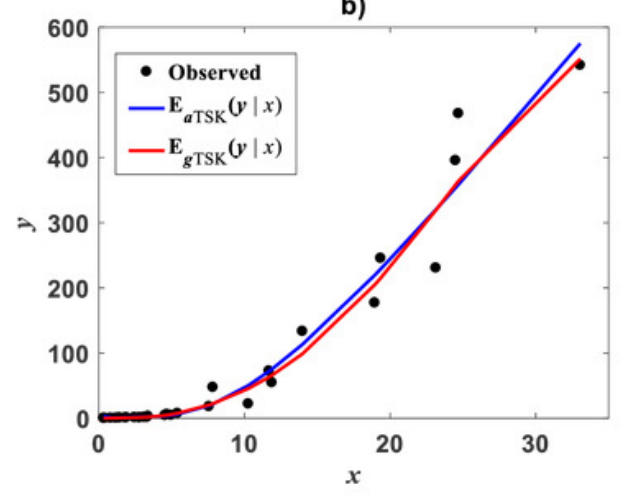

d)

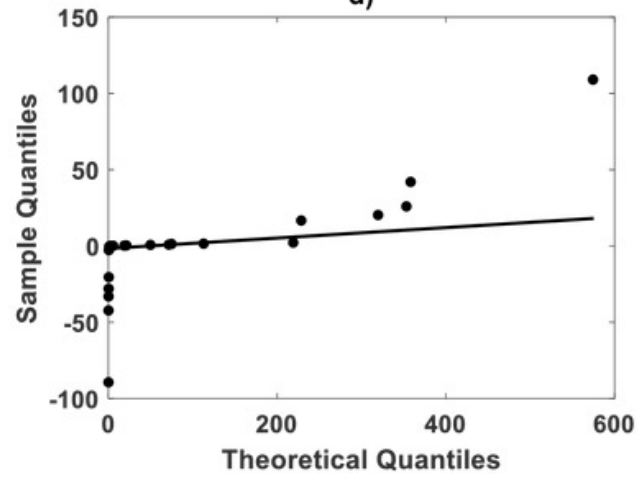

f)

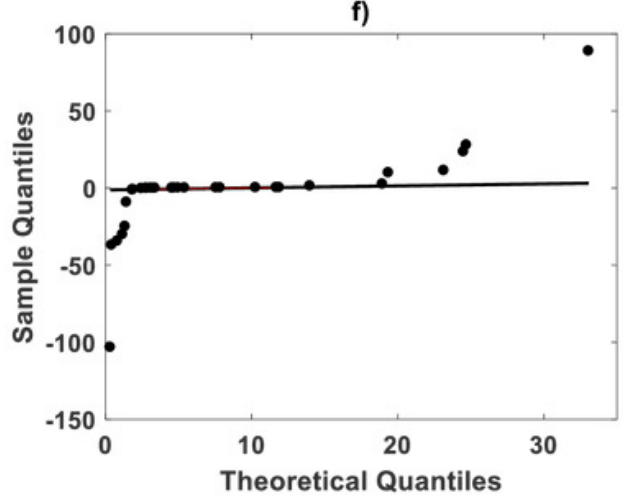




\section{Figure 14}

TSK-MPCA fitted on Huxley (1932) Uca pugnax data.

Panel (a) displays the $q=2$ firing strength factors deriving from an $r_{a}=0.668$ fit of the TSK-

MPCA. A break point places at $x_{b}=340.7$. Panel (b) shows spread about resulting mean function $E_{\text {aTsk }}(y \mid x)$ and compares to $E_{g \text { TรK }}(y \mid x)$ gotten by retransformation of fitted TSK-PLA. Plot suggest corresponding reproducibility strengths. Nevertheless, as it can be made certain by model performance metrics in Table 10, the $E_{g T s k}(y \mid x)$ proxy entails relatively higher reliability. Panel (c) and panel (d) display residual and QQ plots one to one for $E_{\text {aTsk }}(y \mid x)$. Panel (e) and panel (f) show corresponding plots for $E_{g \text { Tsk }}(y \mid x)$. We may be aware that logtransformation based procedures do not lead to biased results for this data. 
a)

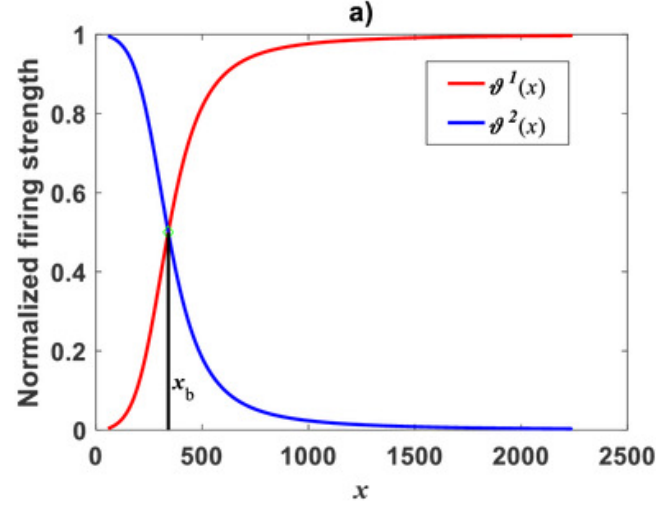

c)

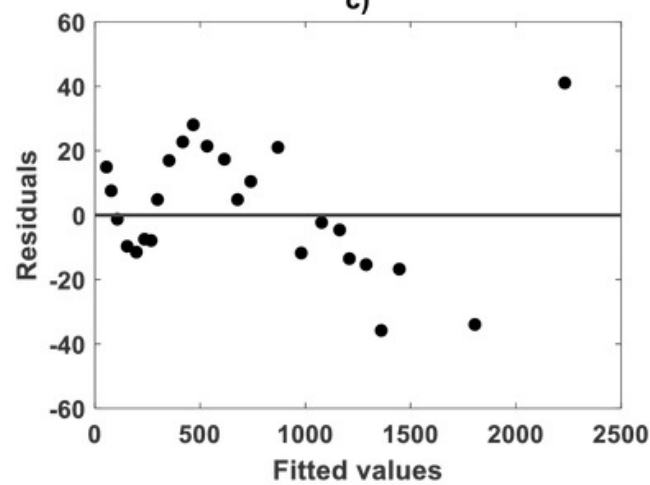

e)

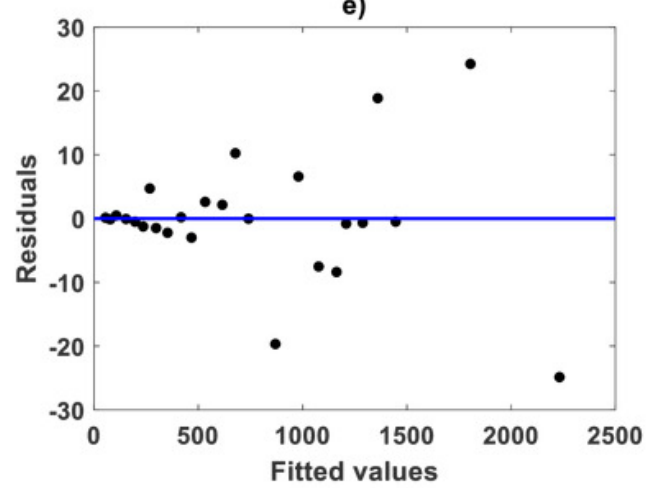

b)

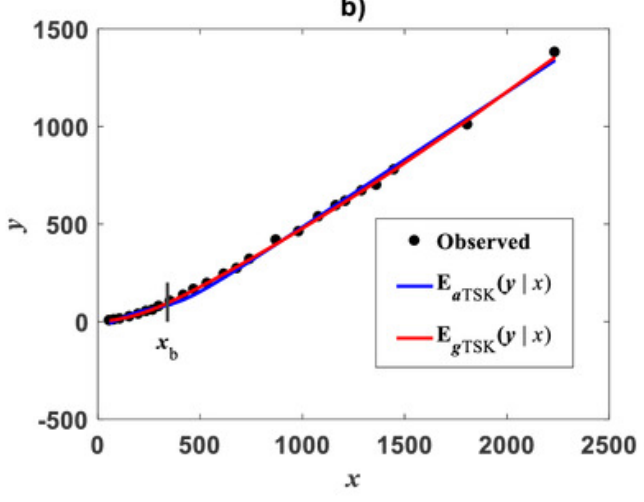

d)

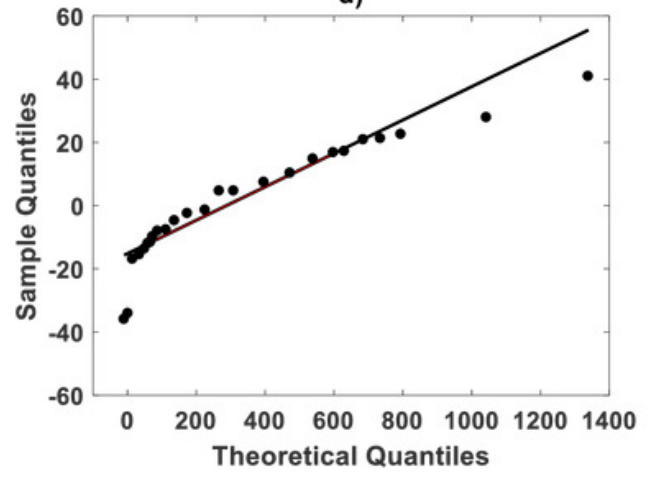

f)

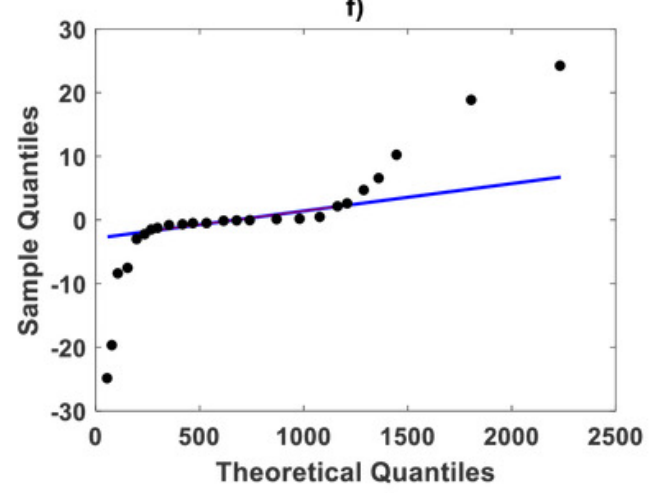




\section{Figure 15}

TSK-MPCA model identified on the De Robertis and Williams (2008) data.

Panel (a) shows spread about the interpolation mean response $E_{\text {aтsk }}(y \mid x)$ produced by a $r_{a}=$ 0.22 and $q=3$ fit of the TSK-MPCA fuzzy model. Plot exhibits a greater adequacy of $E_{g \text { TsK }}(y \mid x)$ gotten by retransformation of output of TSK-PLA fitted to this data. Panel (b) and panel (c) residual spread and QQ plots for $E_{\text {aTsk }}(y \mid x)$ one to one. Panel (d) presents spread about the mean response $E_{a s}(y \mid x)$ produced by a fit of Huxley's formula of simple allometry compared to $E_{g \text { T⿰K }}(y \mid x)$. We can be aware that $E_{g \text { Tsk }}(y \mid x)$ grants similar reproducibility features. Panel (e) and panel (f) residual spread and QQ plots accompanying $E_{a s}(y \mid x)$. 
a)

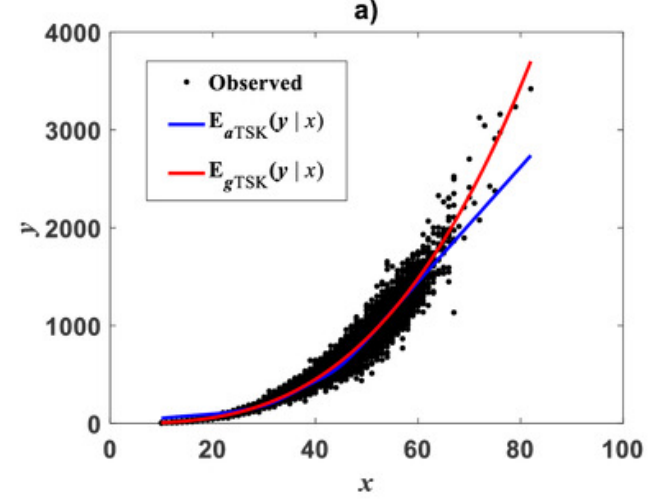

b)

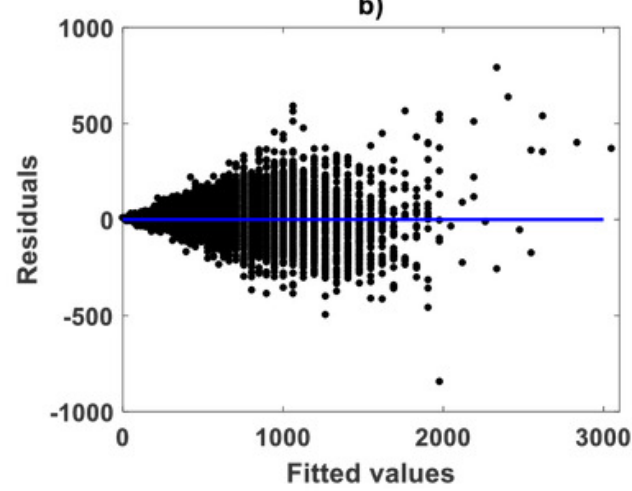

c)

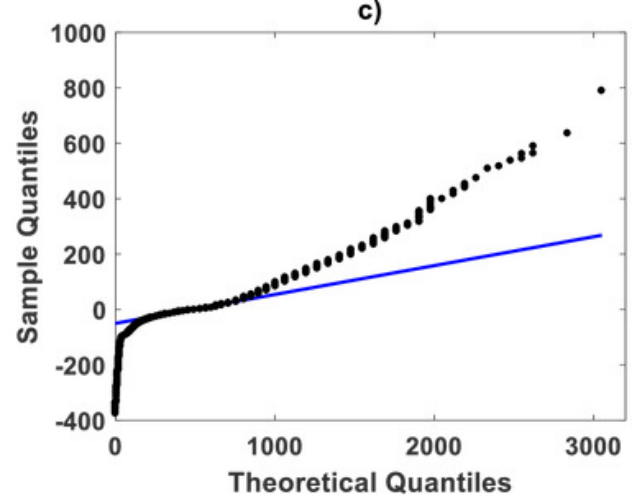

d)

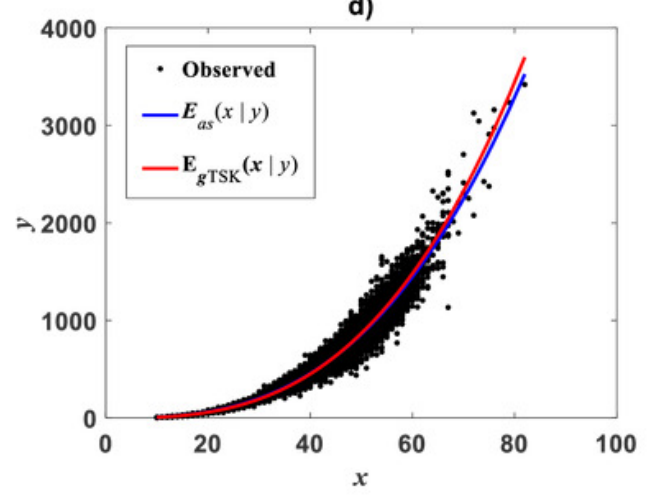

e)

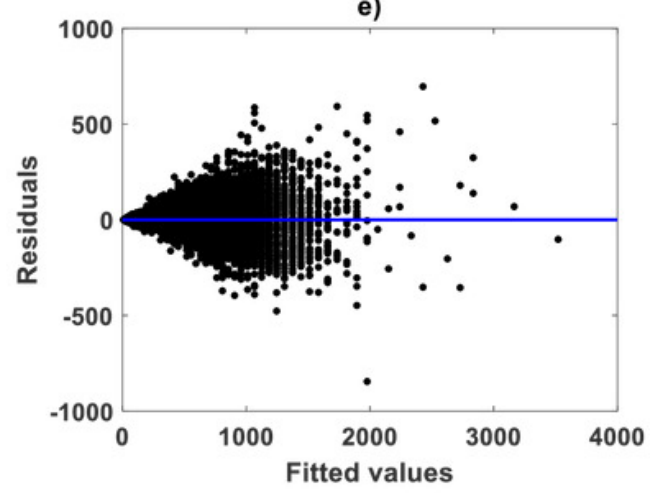

f)

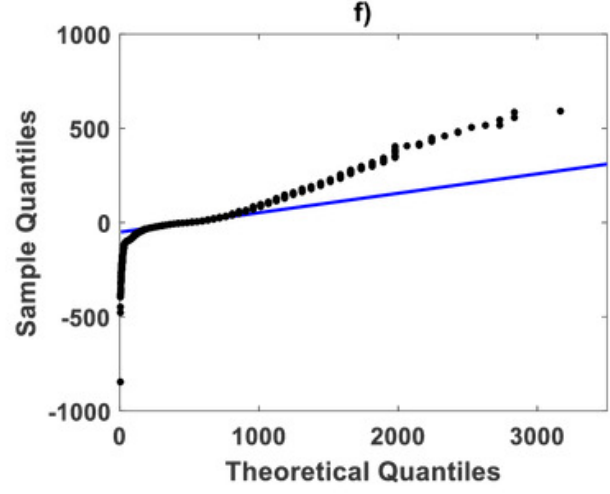




\section{Table $\mathbf{1}$ (on next page)}

Fitting results of the TAMA protocol for the Echavarría-Heras et al. (2019) data set. 


\begin{tabular}{|c|c|c|c|c|c|}
\hline \multicolumn{6}{|c|}{ Residual statistics } \\
\hline Minimum & $1 Q$ & Median & $3 \mathbf{Q}$ & & Maximum \\
\hline-4.7535 & -0.2642 & 0.0042 & 0.21 & & 8.3509 \\
\hline \multicolumn{6}{|c|}{ Coefficient values } \\
\hline Parameters & Estimate & Std. Error & t value & $\operatorname{Pr}(>|t|)$ & Confidence Interval (95\%) \\
\hline \multirow{2}{*}{$\begin{array}{c}\alpha \\
\ln \beta\end{array}$} & 1.022775 & 0.003662 & 279.3 & $<2 \mathrm{e}-16$ & $(1.015597,1.029953)$ \\
\hline & -11.202199 & 0.021515 & -520.7 & $<2 \mathrm{e}-16$ & $(-11.24437,-11.16003)$ \\
\hline \multicolumn{6}{|l|}{ Fitting test. } \\
\hline \multicolumn{3}{|l|}{ Test } & \multicolumn{3}{|l|}{ Value } \\
\hline \multicolumn{3}{|c|}{ Residual standard error } & \multicolumn{3}{|c|}{0.5723 on 10410 degrees of freedom } \\
\hline \multicolumn{3}{|c|}{ Multiple R-squared } & \multicolumn{3}{|c|}{0.8823} \\
\hline \multicolumn{3}{|c|}{ Adjusted R-squared } & \multicolumn{3}{|l|}{0.8823} \\
\hline \multirow{2}{*}{\multicolumn{2}{|c|}{$\begin{array}{l}\text { F-statistic } \\
\text { p-value }\end{array}$}} & & \multicolumn{3}{|c|}{ 7.802e+04 on 1 and $10410 \mathrm{DF}$} \\
\hline & & p-value & \multicolumn{3}{|c|}{$<2.2 \mathrm{e}-16$} \\
\hline
\end{tabular}




\section{Table 2 (on next page)}

Fitting results of the TSK-PLA regression model of equation (17) for the Echavarría-Heras et al. (2019) data set, assuming heteroscedasticity in the form given by equation (35). 
1

\begin{tabular}{|c|c|c|c|c|c|}
\hline \multicolumn{6}{|c|}{ Residual statistics } \\
\hline Minimum & \multicolumn{2}{|l|}{ 1Q } & Median & $3 Q$ & Maximum \\
\hline-4.4478 & \multicolumn{2}{|c|}{-0.2273} & $99 \times 10^{-5}$ & 0.1936 & 7.5718 \\
\hline \multicolumn{6}{|c|}{ Fitting results } \\
\hline Parameters & Estimate & Std. Error & t value & $\operatorname{Pr}(>|\mathbf{t}|)$ & Confidence Interval (95\%) \\
\hline $\ln \beta_{1}$ & -11.7672 & 0.0259 & -454.09 & $<2.2 \times 10^{-16}$ & $(-11.8180613-11.7164792)$ \\
\hline$\alpha_{1}$ & 1.1148 & 0.0036 & 302.84 & $<4.0 \times 10^{-16}$ & $\left(\begin{array}{ll}1.1076198 & 1.1220501)\end{array}\right.$ \\
\hline $\ln \beta_{2}$ & -9.2326 & 0.0803 & -114.93 & $<2.2 \times 10^{-16}$ & $(-9.3901440 \quad-9.0752428)$ \\
\hline$\alpha_{2}$ & 0.3629 & 0.0278 & 13.02 & $<4.2 \times 10^{-39}$ & $\left(\begin{array}{ll}0.3083460 & 0.4175571\end{array}\right)$ \\
\hline$\sigma$ & 2.5063 & 0.0169 & 147.93 & $<2.2 \times 10^{-16}$ & $(2.4731764 \quad 2.5395905)$ \\
\hline$k$ & -0.1580 & 0.0021 & -73.21 & $<2.2 \times 10^{-16}$ & $(-0.1623302-0.1538654)$ \\
\hline
\end{tabular}

2

3 


\section{Table 3 (on next page)}

Fitting results of the TSK-PLA regression model of equation (17) for the Echavarría-Heras et al. (2019) data set, assuming homoscedasticity. 


\begin{tabular}{|c|c|c|c|c|c|}
\hline \multicolumn{6}{|c|}{ Residual statistics } \\
\hline Minimum & $1 Q$ & \multicolumn{2}{|c|}{ Median } & 3Q & Maximum \\
\hline-4.4569 & -0.2264 & \multicolumn{2}{|c|}{0.0017} & 0.1943 & 7.5545 \\
\hline \multicolumn{6}{|c|}{ Fitting results } \\
\hline Parameter & Estimate & Std. Error & t value & $\operatorname{Pr}(>|\mathbf{t}|)$ & Confidence Interval (95\%) \\
\hline $\ln \beta_{1}$ & -11.7029 & 0.0369 & -316.34 & $<2.2 \times 10^{-16}$ & $(-11.7754563,-11.6304399)$ \\
\hline$\alpha_{1}$ & 1.1047 & 0.0058 & 189.14 & $<2.2 \times 10^{-16}$ & $(1.0932778,1.1161734)$ \\
\hline $\ln \beta_{2}$ & -9.1869 & 0.0562 & -163.27 & $<2.2 \times 10^{-16}$ & $(-9.2972775,-9.0767044)$ \\
\hline$\alpha_{2}$ & 0.3437 & 0.0205 & 16.69 & $<7.1 \times 10^{-63}$ & $(0.3033585,0.3840624)$ \\
\hline $\boldsymbol{\sigma}$ & 0.5273 & 0.0036 & 144.30 & $<2.2 \times 10^{-16}$ & $(0.5201765,0.5345015)$ \\
\hline
\end{tabular}

1 


\section{Table 4 (on next page)}

Comparison of model performance metrics for TAMA and TSK-PLA models fitted on the Echavarría-Heras et al. (2019) data set.

Included metrics are: AIC, CCC, $\mathrm{R}^{2}$, SEE, MPE, and MPSE. Het refers to heteroscedastic and Hom to homoscedastic model. 


\begin{tabular}{|c|c|c|c|c|c|c|c|c|}
\hline Method & $r_{a}$ & $q$ & $\overline{A I C}$ & $\rho_{c}$ & $R^{2}$ & SEE & MPE & MPSE \\
\hline$\overline{v_{s}(u, \pi)}$ & $\begin{array}{l}---- \\
\end{array}$ & --- & 17928.42 & 0.9375 & 0.8823 & 0.5723 & -0.2077 & 6.4777 \\
\hline$v_{\mathrm{TSK}}(u, \pi):$ Het & 0.47 & 2 & 16240.77 & 0.9475 & 0.9000 & 0.5276 & -0.1915 & 5.7908 \\
\hline$v_{\mathrm{TSK}}(u, \pi):$ Hom & 0.47 & 2 & 16237.40 & 0.9474 & 0.9000 & 0.5275 & -0.1915 & 5.8064 \\
\hline
\end{tabular}

1

2 


\section{Table 5 (on next page)}

Model performance metrics for TAMA and TSK-PLA models fitted on the Mascaro et al. (2011) data set.

Included metrics are: AIC, CCC, R², SEE, MPE, and MPSE. 


\begin{tabular}{|l|cc|c|c|c|c|c|c|}
\hline \multicolumn{1}{|c}{. Method } & \multicolumn{1}{c}{$\boldsymbol{r}_{\boldsymbol{a}}$} & $\boldsymbol{q}$ & \multicolumn{1}{c}{ AIC } & \multicolumn{1}{c|}{$\boldsymbol{\rho}_{\boldsymbol{c}}$} & $\boldsymbol{R}^{2}$ & \multicolumn{1}{c|}{ SEE } & \multicolumn{1}{c|}{ MPE } & MPSE \\
\hline$v_{S}(u, \pi)$ & ---- & --- & 53.4608 & 0.9767 & 0.9544 & 0.5712 & 10.5825 & 47.7494 \\
\hline$v_{\mathrm{TSK}}(u, \pi)$ & 0.80 & 2 & 23.4700 & 0.9943 & 0.9888 & 0.3200 & 5.9292 & 24.4923 \\
\hline
\end{tabular}

1

2 


\section{Table 6(on next page)}

Model performance metrics for TAMA and TSK-PLA models fitted on the Huxley (1932) Uca pugnax data set.

Included metrics are: AIC, CCC, $\mathrm{R}^{2}$, SEE, MPE, and MPSE. 


\begin{tabular}{|c|ccc|c|c|c|c|c|}
\hline Method & \multicolumn{1}{c}{$\boldsymbol{r}_{\boldsymbol{a}}$} & $\boldsymbol{q}$ & AIC & $\boldsymbol{\rho}_{\boldsymbol{c}}$ & $\boldsymbol{R}^{2}$ & SEE & MPE & MPSE \\
\hline$v_{S}(u, \pi)$ & ---- & ---- & -51.4477 & 0.9986 & 0.9972 & 0.0832 & 0.6519 & 1.5399 \\
\hline$v_{\mathrm{TSK}}(u, \pi)$ & 0.8 & 2 & -97.8184 & 0.9999 & 0.9997 & 0.0301 & 0.2359 & 0.4239 \\
\hline$v_{\mathrm{TSK}}(u, \pi)$ & 0.2 & 7 & -127.57 & 0.9999 & 0.9999 & 0.0166 & 0.1301 & 0.2058 \\
\hline
\end{tabular}

1 


\section{Table 7 (on next page)}

Model performance metrics for TAMA and TSK-PLA models fitted on the De Robertis and Williams (2008) data set.

Included metrics are AIC, CCC, $\mathrm{R}^{2}$, SEE, MPE, and MPSE. 


\begin{tabular}{|c|c|c|c|c|c|c|c|c|}
\hline Method & $\boldsymbol{r}_{\boldsymbol{a}}$ & $q$ & AIC & $\rho_{c}$ & $R^{2}$ & SEE & MPE & MPSE \\
\hline$\overline{v_{s}(u, \pi)}$ & ----- & ---- & -55181 & 0.9940 & 0.9881 & 0.0945 & 0.0185 & 1.274 \\
\hline$v_{\mathrm{TSK}}(u, \pi)$ & 0.5 & 1 & -55169 & 0.9941 & 0.9882 & 0.0946 & 0.0186 & 1.274 \\
\hline
\end{tabular}

1

2 


\section{Table 8(on next page)}

Model performance metrics for TSK-PLA and TSK-MPCA models fitted on the EchavarríaHeras et al. (2019) data set.

Included metrics are: AIC, CCC, R², SEE, MPE, and MPSE. 


\begin{tabular}{|c|c|c|c|c|c|c|c|c|}
\hline Method & $\boldsymbol{r}_{\boldsymbol{a}}$ & $q$ & AIC & $\rho_{c}$ & $R^{2}$ & SEE & MPE & MPSE \\
\hline$E_{a \mathrm{TSK}}(y \mid x)$ & 0.5416 & 2 & -73038.16 & 0.9294 & 0.8678 & 0.007 & 1.0993 & 59.31 \\
\hline$E_{g \mathrm{TSK}}(y \mid x)$ & 0.47 & 2 & -73107.75 & 0.9282 & 0.8688 & 0.007 & 1.0956 & 63.00 \\
\hline
\end{tabular}

1

2 


\section{Table 9 (on next page)}

Model performance metrics for TSK-PLA and TSK-MPCA models fitted on the Mascaro et al. (2011) data set.

Included metrics are: AIC, CCC, $\mathrm{R}^{2}$, SEE, MPE, and MPSE. 


\begin{tabular}{|c|c|c|cc|c|c|c|c|}
\hline \multicolumn{1}{|c}{ Method } & \multicolumn{1}{c}{$\boldsymbol{r}_{\boldsymbol{a}}$} & $\boldsymbol{q}$ & AIC & \multicolumn{1}{c}{$\boldsymbol{\rho}_{\boldsymbol{c}}$} & $\boldsymbol{R}^{\mathbf{2}}$ & SEE & MPE & MPSE \\
\hline$E_{a \mathrm{TSK}}(y \mid x)$ & 0.855 & 2 & 305.32 & 0.9785 & 0.9579 & 35.09 & 15.7416 & 33.14 \\
\hline$E_{a \mathrm{TSK}}(y \mid x)$ & 0.52 & 3 & 308.62 & 0.9749 & 0.9530 & 37.0804 & 16.6307 & 111.49 \\
\hline$E_{\text {gTSK }(y \mid x)}$ & 0.80 & 2 & 314.21 & 0.9720 & 0.9434 & 40.7013 & 18.2547 & 22.31 \\
\hline
\end{tabular}

1 


\section{Table $\mathbf{1 0}$ (on next page)}

Model performance metrics for TSK-PLA and TSK-MPCA models fitted on the Huxley (1932) Uca pugnax data set.

Included metrics are: AIC, CCC, R², SEE, MPE, and MPSE. 


\begin{tabular}{|ccccccc|c|c|c|}
\hline Method & \multicolumn{1}{c}{$\boldsymbol{r}_{\boldsymbol{a}}$} & $\boldsymbol{q}$ & AIC & $\boldsymbol{\rho}_{\boldsymbol{c}}$ & $\boldsymbol{R}^{2}$ & \multicolumn{1}{c|}{ SEE } & \multicolumn{1}{c|}{ MPE } & MPSE \\
\hline $\boldsymbol{E}_{\boldsymbol{a T S K}}(\boldsymbol{y} \mid \boldsymbol{x})$ & 0.668 & 2 & 232.81 & 0.9986 & 0.9972 & 22.42 & 2.52 & 32.35 \\
\hline $\boldsymbol{E}_{\boldsymbol{g} \text { TSK }}(\boldsymbol{y} \mid \boldsymbol{x})$ & 0.8 & 2 & 199.72 & 0.9996 & 0.9993 & 11.56 & 1.30 & 1.8548 \\
\hline $\boldsymbol{E}_{\boldsymbol{g} \text { TSK }}(\boldsymbol{y} \mid \boldsymbol{x})$ & 0.2 & 7 & 160.43 & 0.9999 & 0.9998 & 5.27 & 0.59 & 0.9577 \\
\hline
\end{tabular}

1 


\section{Table 11 (on next page)}

Model performance metrics for Huxley's simple allometry, TSK-PLA and TSK-MPCA models fitted on the De Robertis and Williams (2008) data set.

Included metrics are: AIC, CCC, R², SEE, MPE, and MPSE. 


\begin{tabular}{|c|c|c|c|c|c|c|c|c|}
\hline Method & \multicolumn{1}{c}{$\boldsymbol{r}_{\boldsymbol{a}}$} & $\boldsymbol{q}$ & AIC & $\boldsymbol{\rho}_{\boldsymbol{c}}$ & $\boldsymbol{R}^{\mathbf{2}}$ & SEE & \multicolumn{1}{c|}{ MPE } & MPSE \\
\hline$E_{a s}(y \mid x)$ & ----- & --- & 324010 & 0.9812 & 0.9630 & 60.2367 & 0.1401 & 7.3767 \\
\hline$E_{a \mathrm{TSK}}(y \mid x)$ & 0.22 & 3 & 330531 & 0.9762 & 0.9539 & 67.3087 & 0.1565 & 11.6985 \\
\hline$E_{g_{\mathrm{TSK}}}(y \mid x)$ & 0.50 & 1 & 324613 & 0.9812 & 0.9622 & 60.0856 & 0.1415 & 7.2992 \\
\hline
\end{tabular}

1 